\title{
A Lower Valanginian coral fauna from the South Iberian Palaeomargin (Internal Prebetic, SE Spain)
}

\author{
Hannes Löser, Luis M. Nieto, José Manuel Castro, and Matías Reolid
}

\begin{abstract}
From the Lower Valanginian of the Sierra de Cazorla (Internal Prebetic, SE Spain), a coral fauna is taxonomically described. The fauna encompasses 51 species in 29 genera. One genus and three species are described as new. The most speciesrich are the superfamilies Cyclolitoidea and Stylinoidea. The faunal composition is ambivalent and encompasses typical Jurassic taxa, such as members of the families Amphiastraeidae, Rhipidogyridae, Solenocoenidae and Stylinidae, but also typical Cretaceous elements such as the genera Confusaforma, Floriastrea and Holocoenia (which also have their first occurrence in the Valanginian studied fauna). Four Jurassic genera show a range extension into the Early Valanginian: Alloiteaucoenia, Bilaterocoenia, Hykeliphyllum and Miscellosmilia. Other genera still survived into the Late Valanginian (Placogyra, Rhipidogyra and Solenocoenia) but became extinct. A palaeobiogeographic analysis shows relationships of the studied fauna to the Tithonian and the Kimmeridgian of the northern Tethys on one hand, and the Hauterivian of the Paris Basin and the Puebla Basin (Mexico) on the other. Nineteen species of the studied fauna remained in open nomenclature; the majority of them probably represent new species.
\end{abstract}

Hannes Löser. Universidad Nacional Autónoma de México, Instituto de Geología, Estación Regional del Noroeste, L.D. Colosio s/n, 83000 Hermosillo, Sonora, Mexico. loeser@paleotax.de

Luis M. Nieto. Departamento de Geología y Centro de Estudios Avanzados en Ciencias de la Tierra, Energía y Medio Ambiente (CEACTEMA), Universidad de Jaén, 23071 Jaén, Spain. Imnieto@ujaen.es José Manuel Castro. Departamento de Geología y Centro de Estudios Avanzados en Ciencias de la Tierra, Energía y Medio Ambiente (CEACTEMA), Universidad de Jaén, 23071 Jaén, Spain.

jmcastro@ujaen.es

Matías Reolid. Departamento de Geología y Centro de Estudios Avanzados en Ciencias de la Tierra, Energía y Medio Ambiente (CEACTEMA), Universidad de Jaén, 23071 Jaén, Spain. mreolid@ujaen.es

Keywords: corals; Cretaceous; Spain; taxonomy; new species; new genus

Submission: 17 September 2019. Acceptance: 26 January 2021.

http://zoobank.org/459FC316-81E8-4D6C-AA31-8BA5C6A0AFA4

Löser, Hannes, Nieto, Luis M., Castro, José Manuel, and Reolid, Matías. 2021. A Lower Valanginian coral fauna from the South Iberian Palaeomargin (Internal Prebetic, SE Spain). Palaeontologia Electronica, 24(1):a06. https://doi.org/10.26879/1030

palaeo-electronica.org/content/2021/3292-spanish-valanginian-corals

Copyright: February 2021 Paleontological Society.

This is an open access article distributed under the terms of Attribution-NonCommercial-ShareAlike 4.0 International (CC BY-NC-SA 4.0), which permits users to copy and redistribute the material in any medium or format, provided it is not used for commercial purposes and the original author and source are credited, with indications if any changes are made.

creativecommons.org/licenses/by-nc-sa/4.0/ 


\section{INTRODUCTION}

Upper Jurassic and Lower Cretaceous coral faunas represent a different taxonomic inventory. Late Jurassic, particularly Tithonian, coral faunas were mainly dominated by four superfamilies: Amphiastreoidea, Cyclolitoidea, Montlivaltioidea, and Stylinoidea (Eliášová, 1981, 2008; Kolodziej, 2003, 2015a, 2015b; Kuzmicheva, 2002; Löser and Mori, 2002; Ricci et al., 2018). Berriasian to Valanginian coral faunas are almost unknown on a global scale. Hauterivian coral faunas are different: Amphiastreoidea are almost unknown (they appeared again during the Aptian), Montlivaltioidea and Stylinoidea show a lower number of genera, but the superfamilies Actinastreoidea, Cladocoroidea, Eugyroidea, and Thamnasterioidea appeared or gained diversity (Löser, 2016). For instance, within the superfamily Stylinoidea, the family Rhipidogyridae became extinct, but had a successor with the family Aulastraeoporidae. Within the superfamily Eugyroidea, the family Eugyridae appeared with the Hauterivian. The transition between Jurassic and Cretaceous corals under evolutionary aspects is difficult to decipher for two reasons. The first is related to the unclear stratigraphic dating of the faunas from the Jurassic/ Cretaceous boundary, while the second is associated with the eustatic changes recorded in the Berriasian.

The European Tithonian faunas are often summarised under the name Štramberk-type limestone. Formerly, these limestones were considered Tithonian in age, but more detailed studies revealed that they reached stratigraphically into the Lower Cretaceous (Kolodziej, 2015a). The large limestone blocks in the area of Štramberk (Czech Republic) are clearly dated as Tithonian to Early Berriasian (Vašíček et al., 1994; Vašíček and Skupien, 2016, 2019). The same situation presents the Torinosu Limestone; its assignation to the Tithonian was tentative (e.g., Eguchi, 1951), and it includes older as well as younger sediments (Löser and Mori, 2002; Kakizaki et al., 2012; Ohga et al., 2013). Tithonian and lower Berriasian shallow marine sediments with corals crop-out transitionally in the Crimea area (Arkadiev et al., 2018). Historical reports on coral occurrence in the above mentioned areas may not have precise age assignments. Most faunas are still attributed to a Tithonian age because more recent studies on the geology and stratigraphy were almost not accompanied by studies of the corals. Therefore, the stratigraphic status of most presumed Tithonian coral faunas is uncertain. The second reason for the gap in the knowledge about the faunal changes of corals at the Jurassic/Cretaceous boundary is due to a presumably global sea-level fall that started during the Berriasian, leading to a reduction in the shallow marine platform areas (e.g., Haq, 2014). Faunas sedimented during the Berriasian and Valanginian are rarely preserved because they still became eroded during this time span or during the following transgression that began in the Hauterivian. For this reason, coral faunas from the middle to upper Berriasian and Valanginian are poorly documented.

Tithonian to lower Berriasian coral faunas are not numerous on a global scale, but they are rich in species and well documented. Faunas are reported from the Czech Republic, Poland, Japan (see references above), and Italy (Prever, 1909b; Ricci et al., 2018). The fauna described by Sikharulidze (1979) from Tskhanar in Georgia - published with an Albian age - is clearly older and may reach from the Tithonian into the earliest Cretaceous (Löser, 2005).

For the earliest Cretaceous, the literature reports only a few hermatypic coral faunas. The description of Berriasian corals from Siouf Mt in Tunisia (Beauvais and M'Rabet, 1977) is not very detailed; because of the absence of thin sections, the species inventory is difficult to compare to other faunas. Also, the location of the collection is unknown. The recently described section from Lyalintsi in Bulgaria (Roniewicz, 2008) contains a large number of species and genera. The fauna was reported using thin sections that were available for study. Unfortunately, the coral material is highly recrystallised and, for most specimens, it is even difficult to confirm the genus. Smaller Berriasian faunas are reported from the Crimea Mountains (Arkadiev and Bugrova, 1999; Kuzmicheva, 1963, 2002). Faunas from the Berriasian of the Baingoin County (Xizang Autonomous Region, China) described by Weihua Liao and Jinbao Xia (1994) are stratigraphically not well constrained. The authors established many new species, mainly from solitary corals of the genera Epistreptophyllum, Montlivaltia, and Plesiosmilia, often lacking comparison to existing species. A recently mentioned coral fauna from central Mexico (Zell et al., 2016) is neither rich in coral species nor in coral material at all. The fauna is under investigation but will not yield more than 10 species. Berriasian corals from Switzerland are reported by Baron-Szabo (2018) and Baron-Szabo and Furrer (2018). Both compilations illustrate mainly complete unsectioned specimens. Thin sections were not pre- 
pared. As a consequence, coral genera are difficult to determine, and coral species cannot be separated because they require systematic measurements of corallite dimensions, as well as counts of septa.

Between the upper Tithonian and the base of the Hauterivian, nearly 50 genera became extinct (Löser, 2016). About 25\% of these genera were endemic, and $30 \%$ were restricted to the upper Jurassic (and partly lower Berriasian; Figure 1). The few genera that became extinct between the middle Berriasian and Valanginian are all endemic.

A remarkable sea-level rise at the beginning of the Hauterivian resulted in the formation of large epicontinental seas (e.g., Husinec and Jelaska, 2006; Marzouk and Ben Youssef, 2008; Gréselle and Pittet, 2010). The basal Hauterivian marks the beginning of a faunal recovery and origination of coral associations that were typical for the Early Cretaceous, which persisted until the Albian/Cenomanian boundary (Löser, 2016). About 15 new genera appeared at the base of the Hauterivian (Löser, 2016), while more followed in the Barremian and Aptian. Hauterivian faunas are not distributed worldwide but concentrated in certain areas (Götz et al., 2005). Larger coral faunas are known from Georgia (Sikharulidze, 1985), Jamaica (Löser et al., 2009), Japan (Eguchi, 1951), the Paris Basin (Löser, 2013a), Poland (Morycowa, 1964), and the Ukraine (Kuzmicheva, 1960, 1966, 2002). It seems that the faunas of the San Juan Raya Formation in Puebla (Mexico) - formerly assigned to the Aptian - are stratigraphically older and have to be assigned to the boundary between the Valanginian and the Hauterivian (González León et al., 2015). Another two small coral faunas from the Late Valanginian and Early Hauterivian of SE Spain are described by Löser et al. (2019).

The present fauna is therefore of a particular interest because the relatively high number of coral species found in Lower Valanginian sediments will improve our knowledge of the transition between Upper Jurassic and Lower Cretaceous coral faunas. The fauna was briefly studied by Geyer and Rosendahl (1985), but the specimens shown in the publication were not available for study and measurements were not provided in the publication. It was, therefore, difficult to compare published data with our material. Only some species could be positively identified.

\section{GEOLOGICAL SETTING}

\section{Tectonic Setting and Palaeogeography}

In the Internal Prebetic of the Sierra de Segura (Figure 2.4), the Jurassic-Cretaceous transition is represented by a thick unit of up to $400 \mathrm{~m}$ of the Portlandian limestones, with Purbeck facies that have been defined as the Sierra del Pozo Formation (Vera et al., 1982). Two members have been differentiated in the type section of the Sierra del Pozo Formation (Figure 3; see Vera et al., 1982; Jiménez de Cisneros and Vera, 1993). The lower member is made up of a $325 \mathrm{~m}$ thick succession of subtidal limestones, which evolved upwards into peritidal facies, dated as Tithonian-Berriasian (Figure 3); the peritidal facies of the upper part of the lower member have been extensively studied by Jiménez de Cisneros and Vera (1993) and Anderson (2004). The upper member is $50 \mathrm{~m}$ thick, and it has been dated as uppermost Berriasian to Lower Valanginian (García-Hernández, 1978; Geyer and Rosendahl, 1985; Jiménez de Cisneros and Vera, 1993). The studied coral assemblages come from the upper member of the Sierra del Pozo Formation (Figures 3 and 4).

The upper member is made up of bioclastic calcarenites and reefal limestones (Figures 3 and 4 ). The microfacies of the bioclastic calcarenites are packstones to grainstones with peloids, ooids, oncoids, benthic foraminifera (Pseudocyclammina sp., Everticyclammina sp., and miliolids), algae (Clypeina jurassica, Actinoporella sp., Cayeuxia sp., and Salpingoporella sp.), bacinelloid microbial structures, and undetermined bioclasts. A main feature of these rocks is the presence of coral debris, with an upwards trend in the section characterised by a gradual increase in the content of corals (mainly fragments, Figure 4). The best preserved coral fossils are located in the upper part of this member, where they are associated with ostreids. The calcarenites present a variety of sedimentary structures, ranging from cross-lamination in the lower part of the section to cross-stratification at the top, with several sets within a single bed. The mean strike of the sets is $\mathrm{N}-\mathrm{S}$ and the mean dip is $35^{\circ}$ to the East. Some beds show a marked erosive base. Several conglomerate levels and Nerinea-rich beds are interbedded with the calcarenites (Figures 3 and 4). The conglomerate levels contain well-rounded pebbles with microfacies characteristic of older beds from the same stratigraphic succession. Planar and trough cross-bedding are also present in these rocks, with sets of 1 $\mathrm{m}$ thick. Ferruginous lutitic matrix and calcite 
LÖSER ET AL.: SPANISH VALANGINIAN CORALS

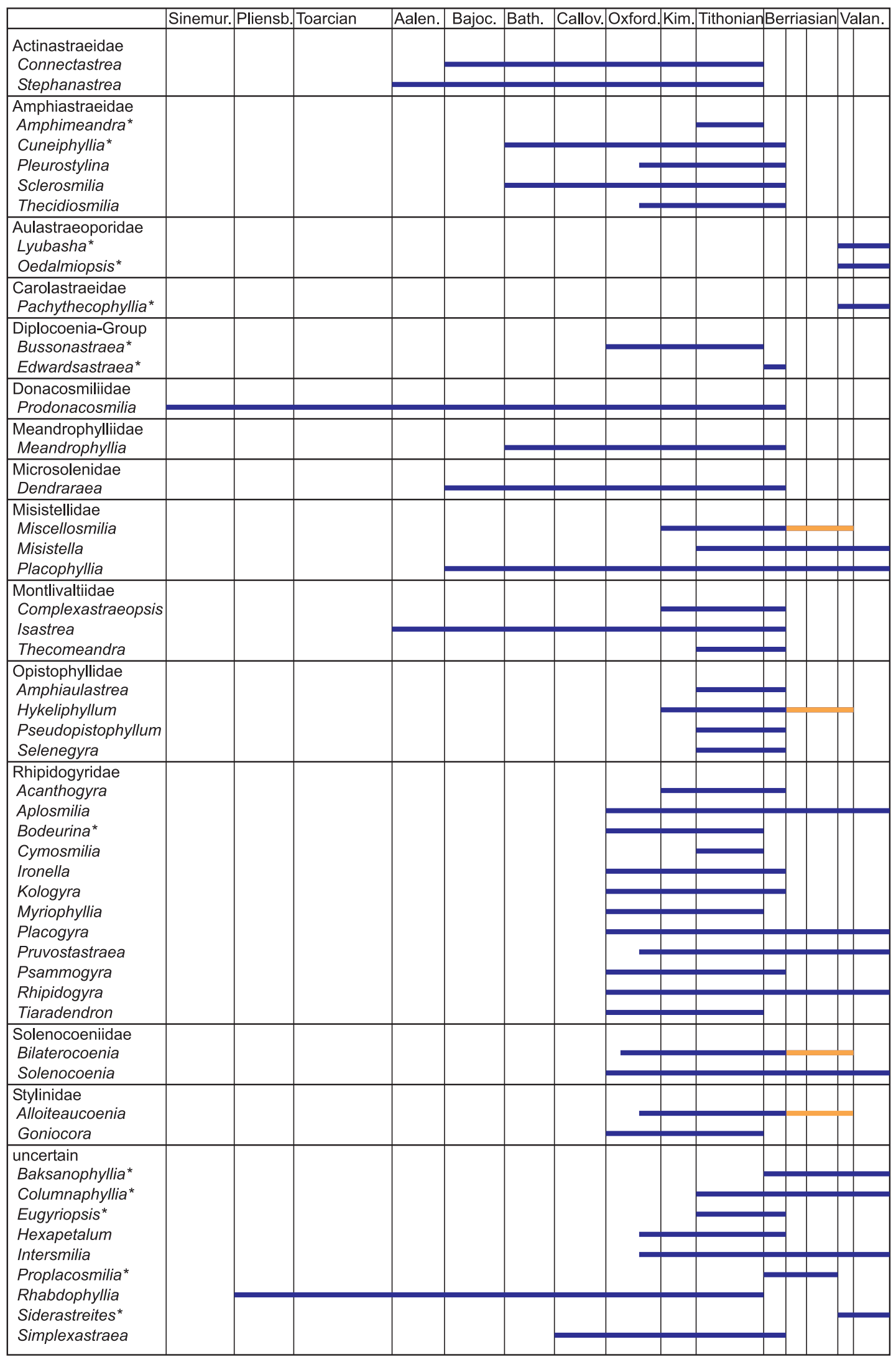

FIGURE 1. Stratigraphical distribution of coral genera that became extinct before the base of the Hauterivian. Orange lines indicate genera that range was extended by the present fauna. 


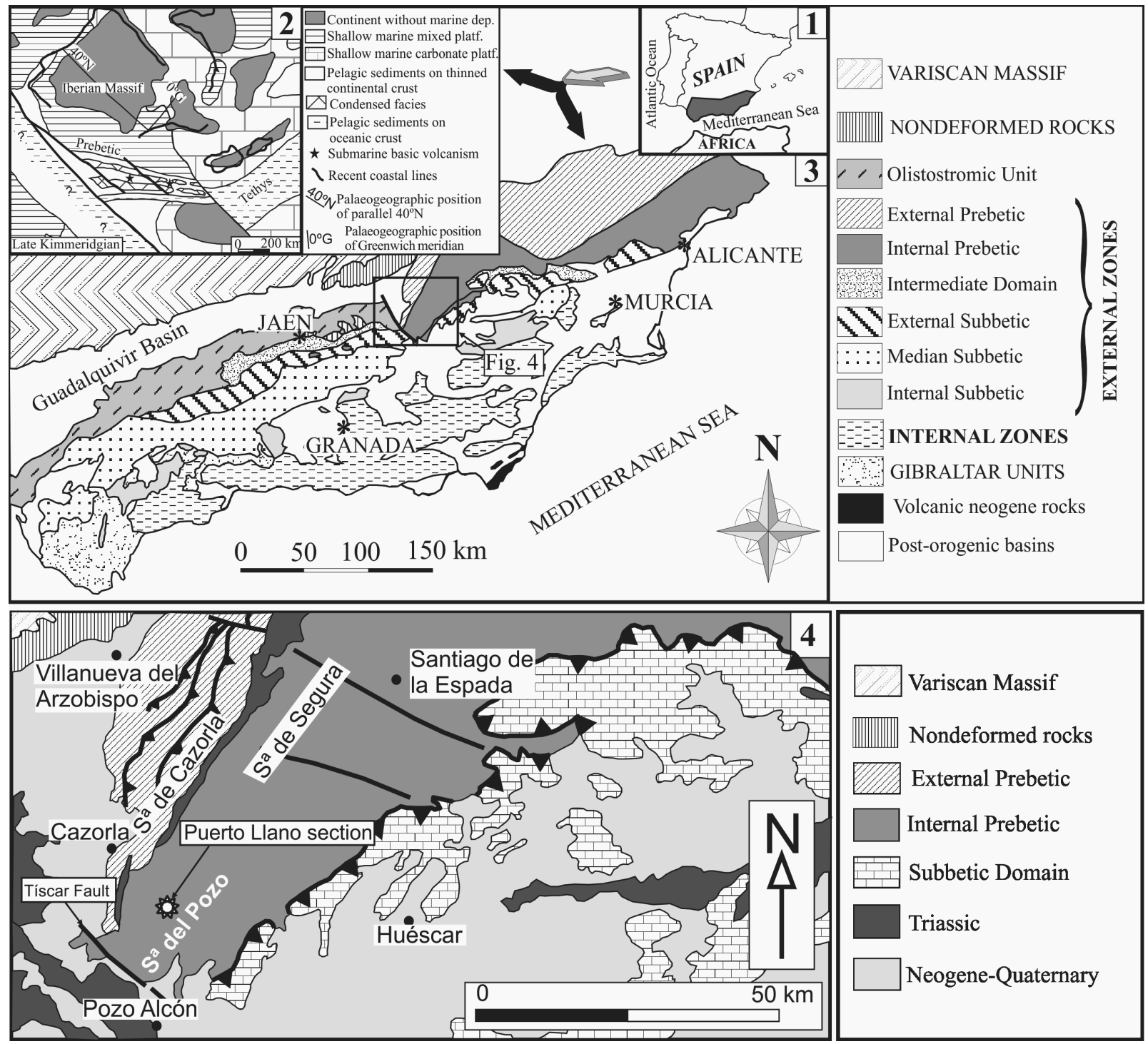

FIGURE 2. Geographical and geological location of the Puerto Llano section. 1. Location of the Betic Cordillera in the South of Spain. 2. Palaeogeographical reconstruction of the South Iberian Palaeomargin in Late Kimmeridgian according to Vera (2001). 3. Geological sketch of the Betic Cordillera with location of the Prebetic zone shown in 4. 4. Geological sketch of the Prebetic in the ended of the Sierra de Cazorla (External Prebetic) and Sierra de Segura (Internal Prebetic) with location of the Puerto Llano section.

cement are present in the calcareous rudites. The quartz grains are abundant in this member, ranging from $5 \%$ to more than $50 \%$. The quartz grains are generally irregular, although rounded grains are also present less frequently. The studied coral assemblage is located at the top of the section (Figures 3 and 4). It consists of a brecciated level with isolated corals and ostreids embedded in a calcarenite matrix, with a peloidal grainstone texture.

\section{MATERIAL AND METHODS}

More than 300 coral specimens were collected from the coral-rich bed at the top of the section, cut and polished. From 70 specimens, a total of 125 thin sections in both transversal and longitudinal orientation were prepared. About 120 specimens were finally included into the taxonomic report. The coral material varies in its state of conservation, with exceptionally well-preserved specimens and others that are strongly re-crystallised or 


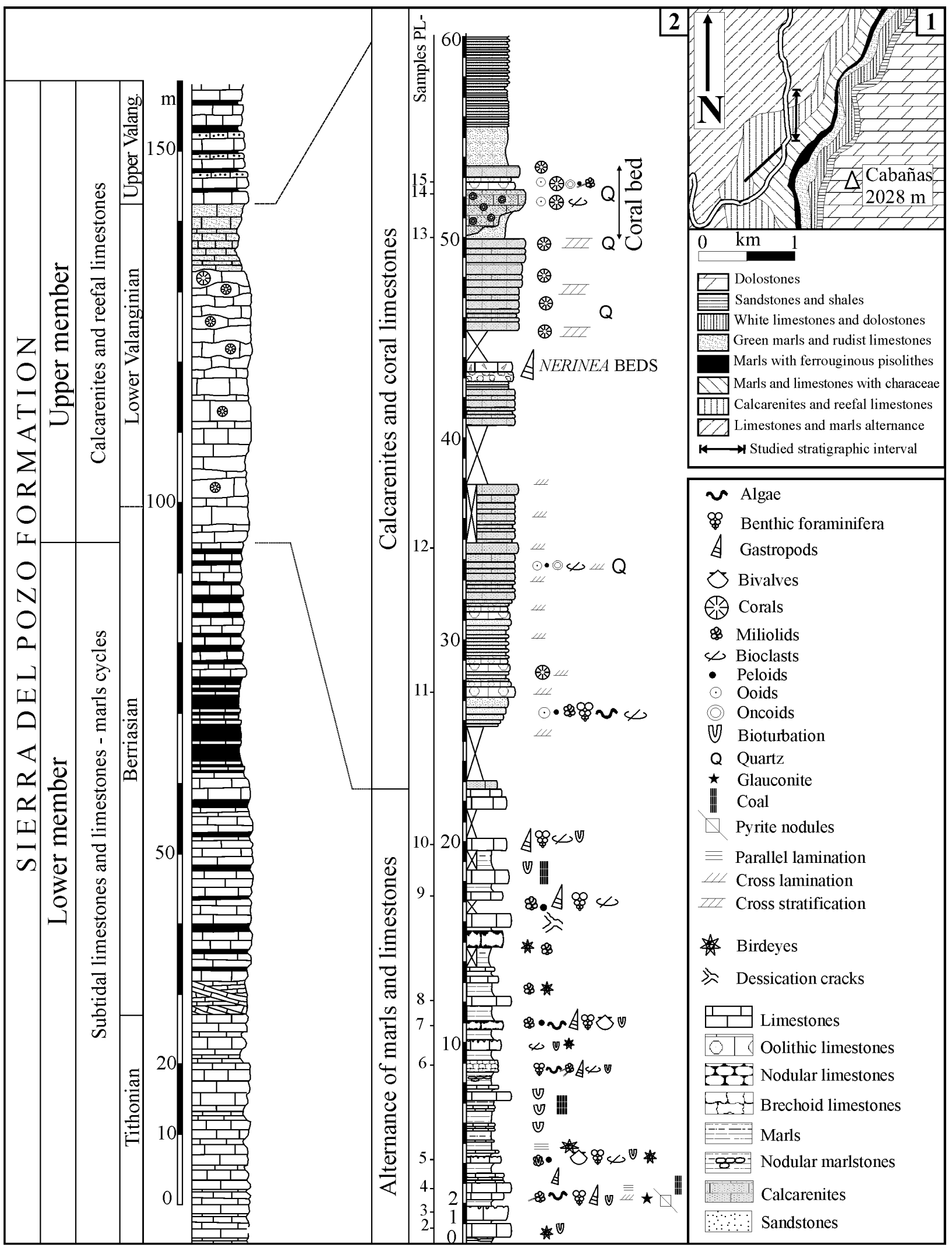

FIGURE 3. Stratigraphic section of the Sierra del Pozo Formation (Tithonian-Valanginian). 1. Detailed geological sketch of the area where the formation crops out. 2. General stratigraphic section of the Sierra del Pozo Formation and detailed section of the upper member, where corals are present. 


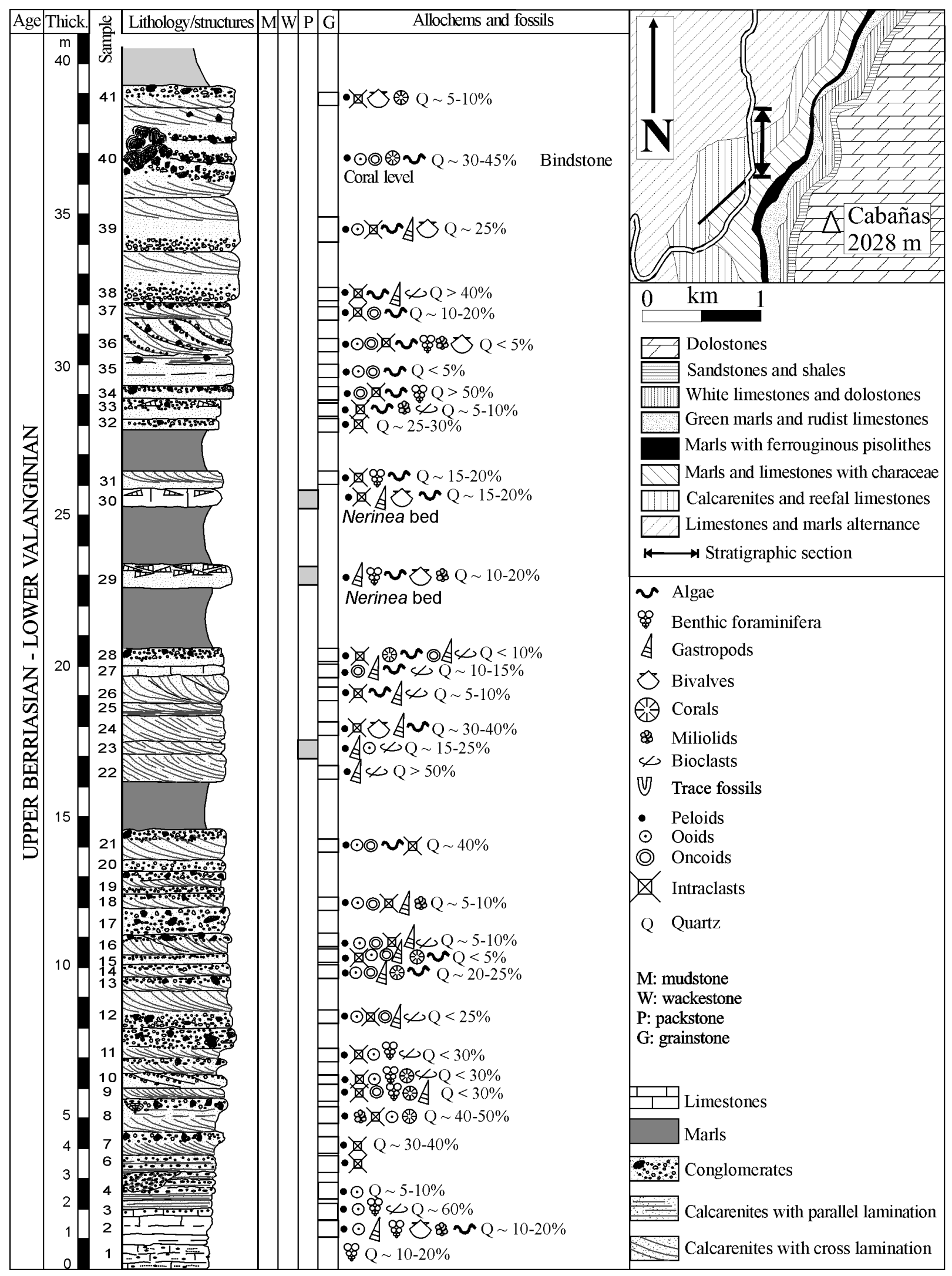

FIGURE 4. Detailed stratigraphic section of the calcarenites and coral limestones (upper member of the Sierra del Pozo Formation) with the position of the samples and their microfacies features. 
fragmented. Smaller colonies were generally better preserved than large ones.

Thin sections were scanned by passing light through them using a flatbed scanner with an optical resolution of $6,400 \mathrm{dpi}$. Scanned images were then transferred to grey scale bit maps. Their quality was amended by histogram contrast manipulation (contrast stretching) where possible.

To gain more insight into the intraspecific variation of fossil corals and to obtain a better strategy for comparing species, corallite dimensions of each specimen were systematically measured. To achieve statistical significance, the largest number of possible measurements was taken. This number was mainly determined by the size and quality of the thin section and the size of the single corallites in relation to the size of the thin sections. Septa were counted for numerous corallites where their symmetry was not regular. For each type of measurement (corallite diameter and distance, width and distance of corallite row) and count (principally septal counts) in one thin section, the following values were obtained:

$$
\begin{aligned}
& \mathrm{n} \text {, number of measurements or counts } \\
& \text { min-max, lowest and highest measured or counted } \\
& \quad \text { values (mm for measurements) } \\
& \mu \text {, arithmetic mean (average) } \\
& \mathrm{s} \text {, standard deviation } \\
& \mathrm{Cv} \text {, coefficient of variation according to K. Pearson } \\
& \mu \pm \mathrm{s} \text {, first interval }
\end{aligned}
$$

Thin sections were measured and values were calculated using the Palaeontological Database System PaleoTax, module PaleoTax/Measure (http://www.paleotax.de/measure); for details on the mathematical background, see Löser (2012b). Morphometric data of the corals were compared against those of specimens in worldwide fossil coral collections, and an associated image database. The database encompasses approximately 26,800 coral specimens from Triassic to modern forms, including 3,850 of Jurassic age and 18,500 of Cretaceous. Approximately 8,150 of them are type specimens, and 15,000 specimens are illustrated. The database is located in the Estación Regional del Noroeste (Instituto de Geología, UNAM), Sonora, Mexico. Data storage and processing were carried out using the PaleoTax database program (Löser, 2004).

To compare the studied fauna with other coral faunas outside the study area, a computer database of about 3,000 worldwide coral localities with coral indications was used (Löser et al., 2002, 2005). To simplify the analysis, localities of the same age, belonging to the same basin, or the same continental margin or the same interoceanic platform, were grouped together into one palaeoprovince (a type of large faunule, sensu Johnson, 2007). Altogether, this produced 470 provinces, reaching from the Jurassic into the Paleogene. Only firmly dated localities were assigned to a province to ensure that the subsequent analysis was valid, and the studied locality was not included in any existing province. For the study area, an independent province was created to allow a clear comparison between the existing provinces and the new material. Interregional comparisons were carried out between the new province and existing ones having at least three species in common with the fauna of the studied area. With few exceptions, the comparisons were carried out using specimens, not the indications in the literature. The literature does not offer precise morphometric data that are necessary to separate species and to compare them to each other. For details, see also Löser (2008) and Löser and Minor (2007). Data analysis, statistics, and the creation of charts were carried out using the Database System PaleoTax and the graphic module PaleoTax/Graph (www.paleotax.de). The material is kept in the Geological Museum of Barcelona (Spain; MGB) under the numbers 83226-83391.

\section{SYSTEMATIC PALAEONTOLOGY}

The abbreviations used in the synonymy lists follow Matthews (1973): *: earliest valid publication of the species name; ?: the assignation of this description to the species is doubtful (so marked quotations are not reflected in the stratigraphic and palaeobiogeographic distribution); $p$ : the described material belongs only in part to the species concerned; v: the specimen was observed by the author. A point before the year indicates a sure reference. A year in italics indicates that the quotation is provided with neither a description nor an illustration.

The distribution data (as reflected in the synonymy lists) are almost entirely based on wellexamined material. Material only mentioned in the literature and material not available or insufficiently described and illustrated in the literature were not taken into account. To obtain better insight into the distribution patterns of the coral fauna of the present fauna, additional unpublished material - indicated by a collection acronym and sample number in parenthesis - has been included. Therefore, distribution data indicated under 'Other occurrences' could also be provided for species remaining in open nomenclature. 


\section{Order Scleractinia}

As explained in previous publications (Löser et al., 2018; Löser and Heinrich, 2018), the classification of the order Scleractinia into suborders is neither practical nor possible. It was therefore proposed to apply superfamilies in place of suborders (Löser, 2016). Twenty-seven superfamilies with 56 families (or informal groups) are distinguished that have a range in the Cretaceous. In contrast to former classification systems based on suborders, the superfamilies may constitute monophyletic groups. The relationships between former suborders and superfamilies, as far as possible, are summarised in a table published in Löser (2016, fig. 5.1.1.7) and in Löser, Steuber and Löser (2018, fig. 7). The basic characteristics for the distinction of the superfamilies are the relative size of the trabeculae, in the ratio to the septa. Further distinction is made based on the presence or absence of synapticulae and the septal perforation, following traditional concepts proposed by Alloiteau (1952) and later authors.

The description of known taxa has been kept short. In Scleractinian corals, superfamilies, families, and genera are distinguished on the basis of qualitative characteristics whereas species are distinguished by quantitative characteristics. The description of two species of the same genus is therefore almost identical because the distinction of species is based on morphometric data such as corallite dimensions and septal counts.

Collection abbreviations are as follows:

ABP, Coll. Armin Bauer, Pressath, Germany;

BSPG, Bayerische Staatssammlung für Paläontologie und Geologie, München, Germany;

BUFGG, Bukarest University, Faculty of Geography and Geology, Bucharest, Romania;

CAMSM, The Sedgwick Museum of Earth Sciences, Cambridge, UK;

$\mathrm{CF}, \mathrm{C}$. Fricot, private collection, Esclarolles-Lurey, France;

CGS, Ceská geologická sluzba, Praha, Czech Republic;

ERNO, Universidad Nacional Autónoma de México, Instituto de Geología, Estación Regional del Noroeste, Hermosillo, Mexico;

FGUB, Facultad de Geología de la Universidad de Barcelona, Spain;

FLH, Coll. Fritz Lang, Hirschaid, Germany;

FSL, Université Claude Bernard, Institut de Géologie, Lyon, France;

GPSL, Geologische und Paläontologische Sammlung der Universität Leipzig, Germany;

HJGL, Hans-Jürgen Gawlick, Leoben, Austria;
IGM, Instituto de Geología, Mexico City, Mexico;

LFU, Landesamt für Umwelt, München, Germany;

$M B$, Museum für Naturkunde der Humboldt-Universität, Berlin, Germany;

MGB, Museu de Geología de Barcelona, Spain;

MGL, Musée Géologique, Lausanne, Switzerland;

MGSB, Museo Geológico del Seminario de BarceIona, Spain;

MGU, Muzej zemlevedenia Moskovskogo Gosudarstvennogo Universiteta, Moskva, Russia;

MHE, Matthias Heinrich, Eckental, Germany;

MHNG, Muséum d'histoire naturelle de la Ville de Genève, Switzerland;

MHNN, Muséum d'Histoire naturelle de Neuchâtel, Switzerland;

MJSN, Musée jurassien des Sciences naturelles, Porrentruy, Switzerland;

MNHN, Muséum National d'Histoire Naturelle, Paris, France;

NHM, The Natural History Museum, London, UK;

$\mathrm{NMNH}$, National Museum of Natural History, Washington, D.C., USA;

NSM, The National Science Museum, Tokyo, Japan;

OKSB, Coll. O. Karousek, Stara Boleslav, Czech Republic;

PU, Museo di Geología e Paleontologia dell' Università di Torino, Italy;

RUB, Ruhr-Universität, Geologisches Institut, Bochum, Germany;

SGM, Coll. Moosleitner, Salzburg, Austria;

SMNS, Staatliches Museum für Naturkunde, Stuttgart, Germany;

TMM, Texas Memorial Museum, Austin, Tex., USA;

TUM, The Tohoku University Museum, Sendai, Japan;

TUMIG, Technische Universität München, Ingenieurgeologie, Germany;

UJ, Jagiellonian University, Instytut Nauk Geologicznych, Kraków, Poland;

UJDE, Universidad de Jáen, Departamento de Geología, Jaén, Spain;

UP, Université de Provence, Marseille, France;

UPS, Université Paul Sabatier, Laboratoire de Géologie Sédimentaire et Paléontologie, Toulouse, France;

ZPAL, Polish Academy of Sciences, Institute of Paleobiology, Warszawa, Poland.

The following abbreviations are used describing the dimensions of the corals:

c, calicular diameter (outer diameter);

ccd, distance between calicular centres;

$c d w$, distance between calicular centres within calicular series; 

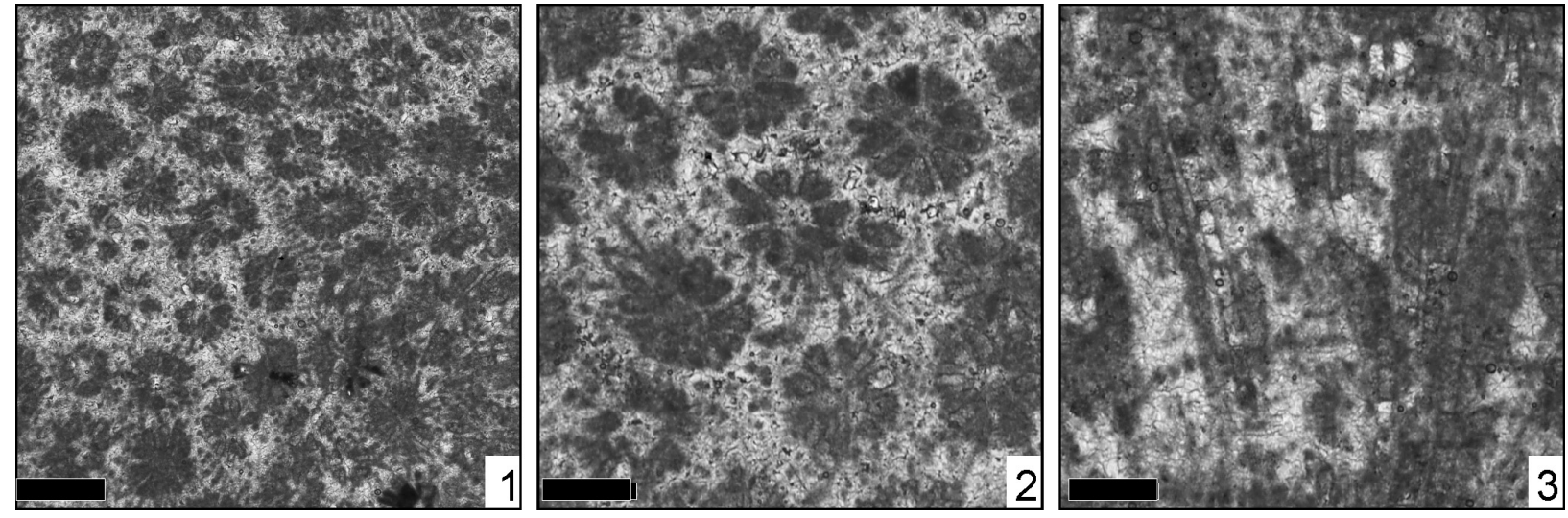

FIGURE 5. ?Actinastrea sp., MGB 83276. 1: transversal thin section. 2: transversal thin section, detail. 3: longitudinal thin section. Scale $1 \mathrm{~mm}$.

clmax, large lumen;

clmin, small lumen;

cmax, larger outer calicular diameter;

cmin, smaller outer calicular diameter;

crd, distance of calicular series;

$\mathrm{s}$, number of septa in the adult corallite;

sd, density of septa.

Phylum CNIDARIA Milne Edwards, 1857

Class ANTHOZOA Ehrenberg, 1834

Superfamily ACTINASTREOIDEA Alloiteau, 1952

Family ACTINASTREIDAE Alloiteau, 1952

Genus ACTINASTREA Orbigny, 1849

Type species. Actinastrea goldfussi Orbigny, 1850 , by subsequent designation.

\section{Actinastrea sp.}

\section{Figure 5}

Material. MGB 83276; 1 thin section.

Dimensions. See Table 1.

Remarks. The genus Actinastrea was revised by Löser (2012a). Generally, it only occurs in the Upper Cretaceous. Actinastrea differs from the similar Stelidioseris by the presence of large isolated trabeculae in the intercalicular space. In Stelidioseris this space is occupied by short confluent costae. The present specimen shows all the characteristics of Actinastrea. Because of the long stratigraphic gap, the present material is here assigned to this genus with reservation.

TABLE 1. Measurements of MGB 83276.

\begin{tabular}{ccccccc}
\hline & $\mathbf{n}$ & $\mathbf{m i n}-\mathbf{m a x}$ & $\boldsymbol{\mu}$ & $\mathbf{s}$ & $\mathbf{c v}$ & $\boldsymbol{\mu \pm s}$ \\
\hline clmin & 30 & $1.04-1.33$ & 1.16 & 0.08 & 7.5 & $1.07-1.25$ \\
clmax & 30 & $1.17-1.82$ & 1.41 & 0.14 & 10.5 & $1.26-1.56$ \\
ccd & 30 & $1.42-1.78$ & 1.61 & 0.09 & 5.9 & $1.51-1.70$ \\
$\mathrm{~s}$ & $8+8$ & & & & & \\
\hline
\end{tabular}

Floriastrea Löser, Stemann, and Mitchell, 2009

Type species. Floriastrea planinensis Turnšek and Mihajlovic, 1981, by original designation.

Floriastrea iberica sp. nov. Löser

Figure 6

zoobank.org/D708B41C-0000-4C9A-AC7D-F98B8A6C3BC2

1985

Latusastrea exiguis (de Fromentel, 1862); Geyer and Rosendahl, p. 167, pl. 2, fig. 6

Etymology. Iberia for the Spanish Peninsula.

Holotype. MGB 83251 with two thin sections, here Figure 6.1, 6.2, 6.4.

Paratypes. MGB 83242 with one thin sections, 83314 with two thin sections, here Figure 6.3.

Type locality. Puerto Llano section, Cabañas, Sierra de Cazorla, Jaén, Andalusia, Spain.

Type level. Sierra del Pozo Fm, Lower Valanginian.

Depository. Museo de Geología de Barcelona, Spain.

Diagnosis. Floriastrea with very small corallites: 0.43-0.52 $\mathrm{mm}$ for the inner smaller and 0.48-0.63 $\mathrm{mm}$ for the inner larger diameter. The number of septa is 10 to 12 .

Comparison. Both known species have larger corallite dimensions. Floriastrea planinensis (Turnšek and Mihajlovic, 1981): clmin, 0.6-0.8 mm; clmax, 0.9-1.1 mm. Floriastrea sexradiata (Sikharulidze, 1985): clmin, 1.3-1.5 mm; clmax, 1.3-1.5 mm.

Material. MGB 83238, 83242, 83251, 83252, $83253,83259,83280,83285,83312,83314 ; 6$ thin sections.

Dimensions. See Table 2.

Description. Plocoid colony where corallites are arranged in a circular pattern. Corallite outline circular to elliptical. Septa compact. Microstructure of septa unknown. Septa in cross section externally thick, then equally very thin. Septal maximum 

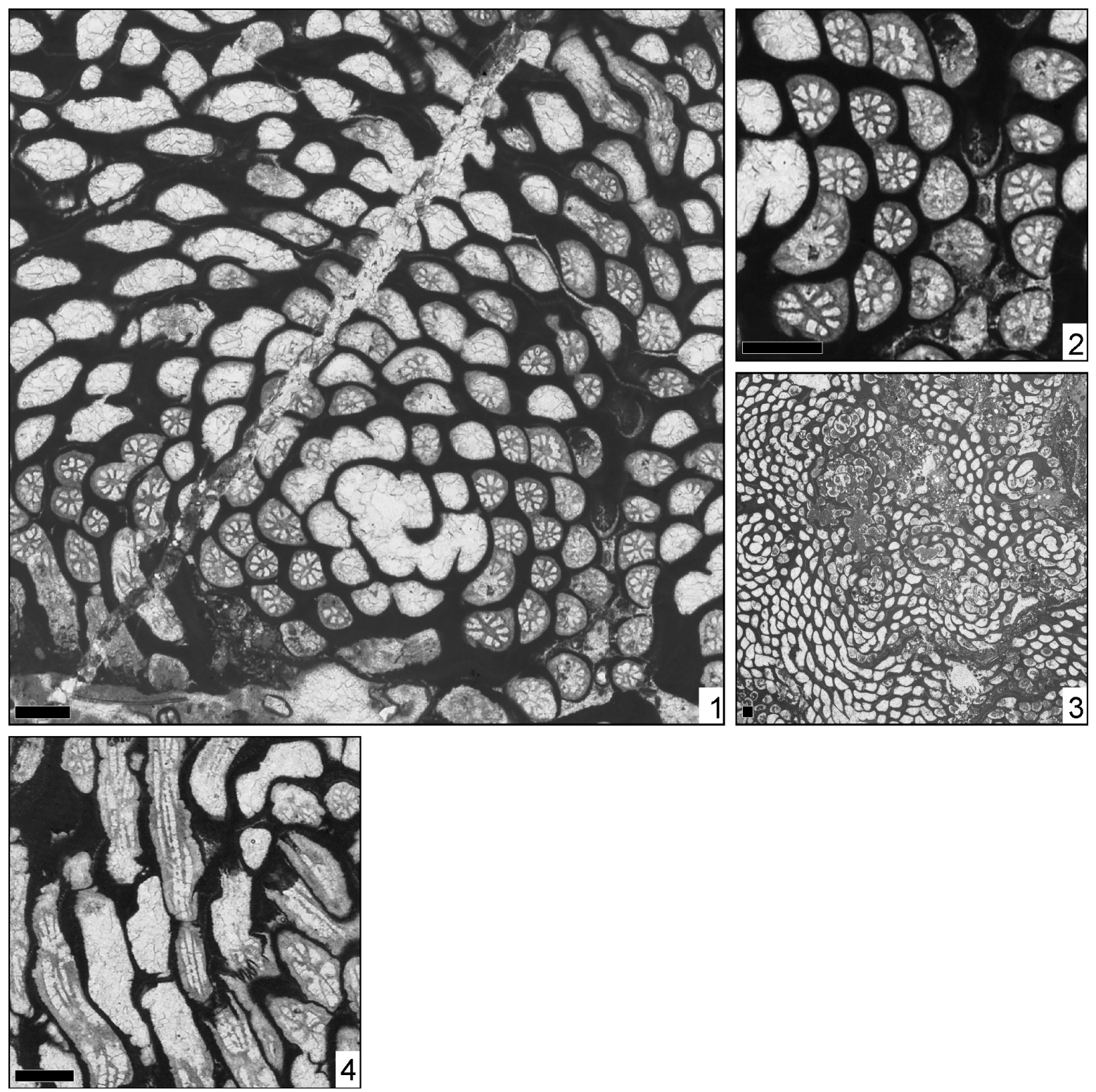

FIGURE 6. Floriastrea iberica sp. nov., Löser. 1: MGB 83251, transversal thin section. 2: MGB 83251, transversal thin section, detail. 3: MGB 83314, transversal thin section, larger view. 4: MGB 83251, longitudinal thin section. Scale 1 $\mathrm{mm}$.

thickness $90 \mu \mathrm{m}$. Symmetry of septa radial in an octameral symmetry. First septal cycle and the beginning of a second cycle resulting in 10 to 12

TABLE 2. Measurements of MGB 83251.

\begin{tabular}{ccccccc}
\hline & $\mathbf{n}$ & $\min -\mathbf{m a x}$ & $\boldsymbol{\mu}$ & $\mathbf{s}$ & $\mathbf{c v}$ & $\boldsymbol{\mu \pm s}$ \\
\hline clmin & 40 & $0.40-0.56$ & 0.48 & 0.04 & 9.2 & $0.43-0.52$ \\
clmax & 40 & $0.46-0.76$ & 0.56 & 0.07 & 13.3 & $0.48-0.63$ \\
ccd & 40 & $0.60-0.92$ & 0.77 & 0.08 & 11.3 & $0.68-0.86$ \\
$\mathrm{~s}$ & 10 & $10-13$ & 11.10 & 1.19 & 10.7 & $10-12$ \\
\hline
\end{tabular}

septa. Septal cycles differ in length. First septal cycle extends to the corallite centre, the second cycle is very short. Septa of the second cycle often attached to those of the first cycle. Septal distal margin unknown, lateral face smooth, inner margin smooth. Pali absent. All septa of the first cycle are attached to the columella. Costae hardly present, non-confluent. Synapticulae absent. Columella styliform. Endotheca consists of thin tabulae. Wall present, compact, probably paraseptothecal. Coenosteum very narrow (rarely more than 100 $\mu \mathrm{m})$. Constitution of the coenosteum unknown. Budding intracalicinal. 

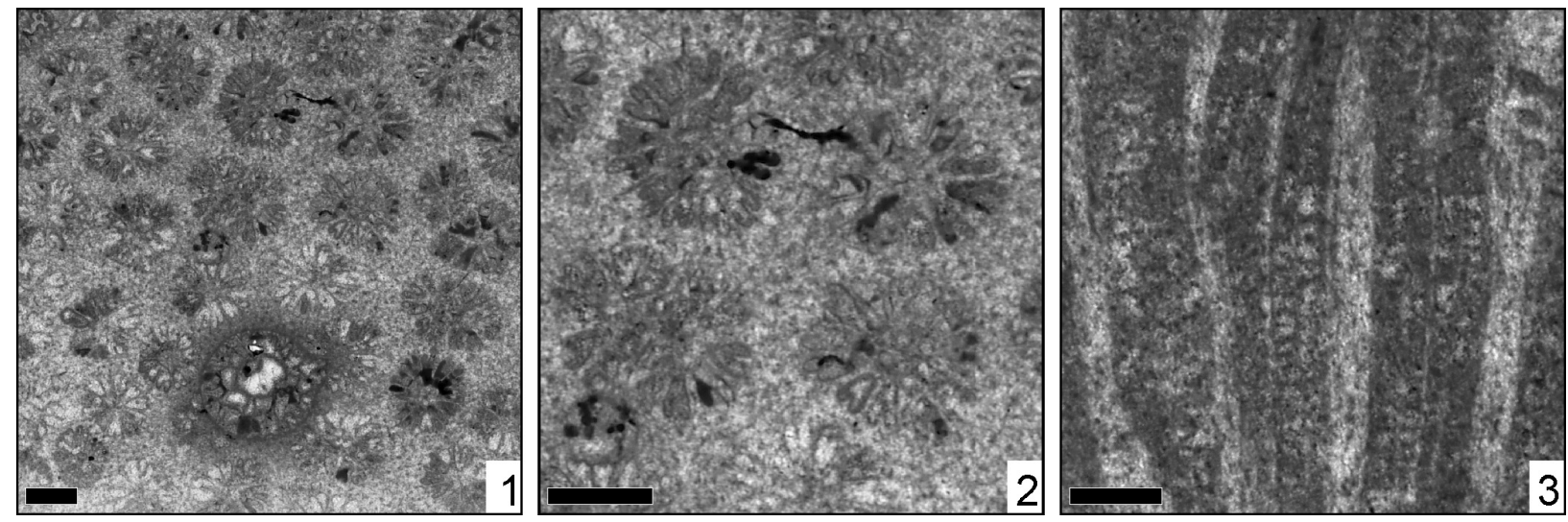

FIGURE 7. Stelidioseris melkarthi Felix, 1909, MGB 83295. 1: transversal thin section. 2: transversal thin section, detail. 3: longitudinal thin section. Scale $1 \mathrm{~mm}$.

Stelidioseris Tomes, 1893

Type species. Stelidioseris gibbosa Tomes, 1893, by monotypy.

Stelidioseris melkarthi (Felix, 1909)

Figure 7

v 1893 Astrocoenia tourtiensis, Bölsche; Tomes, p. 571 , pl. 20, fig. 3

v*1909 Stephanocoenia melkarthi Felix, p. 174, pl. 7, fig. 9

v1926 Astrocoenia bernensis f. sphaeroidalis; Dietrich, p. 92, pl. 6, fig. 2, pl. 7, fig. 5, pl. 10, figs. 2, 3

v 1936 Astrocoenia ex. aff. aequibernensis n. sp.; Hackemesser, p. 75, pl. 8, figs. 10, 11

v 1981 Heliocoenia actinastrae n.sp.; Turnšek and Mihajlovic, p. 11, pl. 2, figs. 1-4, pl. 3, fig. 1, 2

v1989 Actinastraea cf. pseudominima (Koby 1896); Löser, p. 98, text-fig. 3, pl. 21, fig. 3

v1989 Heliocoenia ? actinastrae Turnsek 1981; Löser, p. 108, text-figs. 14, 15, pl. 21, figs. 7, 8

v 1994 Actinastrea actinastrae (Turnsek 1981); Löser, p. 6, text-figs. 2, 3, pl. 5, figs. 1, 2

v1996 Actinastrea actinastrae (Turnsek, 1981); Baron-Szabo and Steuber, p. 6, pl. 1, fig. 1

v2003 Actinastrea aff. pseudominima (Koby, 1897); Baron-Szabo, Hamedani and Senowbari-Daryan, p. 201, pl. 36, figs. 5, 6

v 2008 Actinastrea kunthi (Bölsche, 1871); Löser, p. 38, pl. 1, fig. 5

v 2012 Stelidioseris actinastrae (Turnšek, 1981); Bover Arnal, Löser and Moreno Bedmar, p. 55, fig. 9CD

v 2013b Stelidioseris whitneyi (Wells, 1932); Löser, fig. 3d-f

v 2014 Stelidioseris melkarthi (Felix, 1909); Löser, p. 21 , fig. $2 b$
TABLE 3. Measurements of MGB 83295.

\begin{tabular}{ccccccc}
\hline & $\mathbf{n}$ & $\mathbf{m i n}-\mathbf{m a x}$ & $\boldsymbol{\mu}$ & $\mathbf{s}$ & $\mathbf{c v}$ & $\boldsymbol{\mu \pm \mathbf { s }}$ \\
\hline clmin & 15 & $1.07-1.59$ & 1.25 & 0.15 & 12.1 & $1.10-1.41$ \\
clmax & 15 & $1.43-1.89$ & 1.71 & 0.14 & 8.6 & $1.56-1.86$ \\
ccd & 18 & $1.12-1.93$ & 1.60 & 0.23 & 14.7 & $1.36-1.84$ \\
$\mathbf{s}$ & $6+6+12$ & & & & & \\
\hline
\end{tabular}

Material. MGB 83295; 3 thin sections.

Dimensions. See Table 3.

Remarks. The genus Stelidioseris was recently revised (Löser, 2012a); the species described in Löser (2014).

Occurrence. Jurassic of South Africa, Grignaland, E bank of Vaal River, Steinkops River, Daniels Kuil (NHM R30956). Callovian to Kimmeridgian of Japan (Kochi-ken) Takaoka-gun, Sakawa-cho, Kamo, Mitoda (TUM 65363). Upper Oxfordian of France, Bourges (MNHN nn); France (HauteSaône) Gray, Roche-sur-Vannon (MNHN nn). Kimmeridgian of USA (Texas) EI Paso County, Malone Mts (NMNH I-74242). Lower Kimmeridgian of Poland (Swietokrzyskie) Holy Cross Mts, Baltów (ZPAL Hiii1326). Upper Kimmeridgian to Lower Tithonian of Tanzania (Tanganyika, Mtwara) Mtshinyiri river bank, Karani Kumihu (MB K1310); Tingutinguti River. Cretaceous of Greece (Fokída) Kiona massif, Panourgias. Lower Hauterivian (Radiatus Zone) of France (Yonne) Fontenoy, field $S$ the junction to Les Merles (BSPG 2003 XX 5172). Barremian of France (Doubs) Morteau (MHNN 26754). Upper Barremian of France (Ardèche) St. Remèze, Pont de Laval (BSPG 2003 XX 5220). Upper Barremian to Lower Aptian (Lenticularis Zone) of Mexico (Sonora) Ures, Cerro de Oro (ERNO 2145). Lower Aptian of Greece (Viotía) Arachova; Poland (Malopolskie, Wadowice) Lanck- 

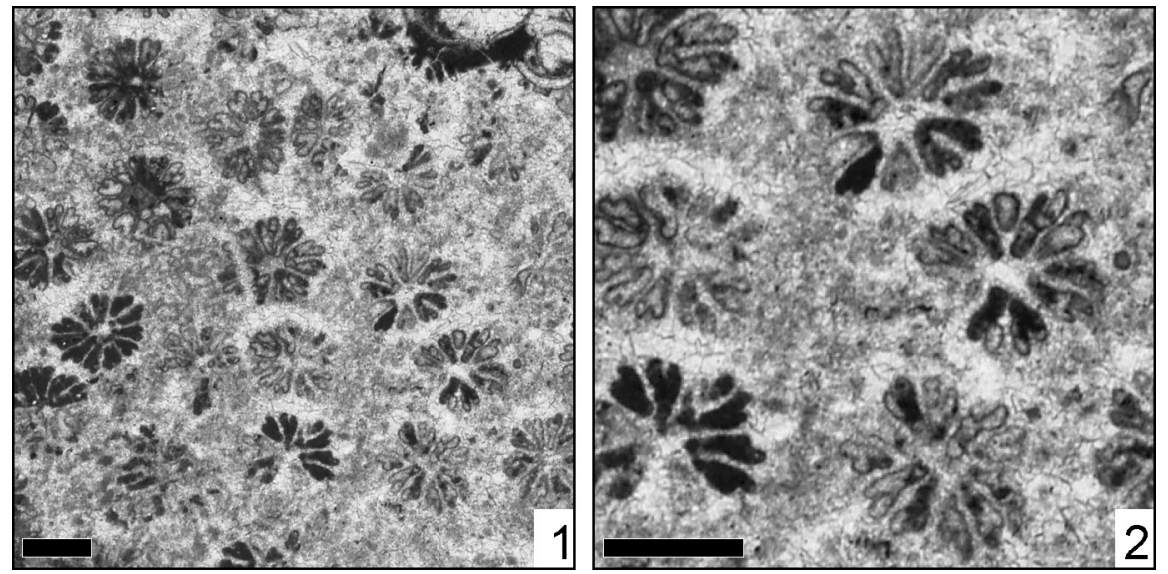

FIGURE 8. Stelidioseris sp., MGB 83270. 1: transversal thin section. 2: transversal thin section, detail. Scale $1 \mathrm{~mm}$.

orona, Jastrzebia (BSPG 2003 XX 5420); Lebanon (Beiroût) Beiroût, Meirouba, Sannin Mt; Serbia (East Serbia) Zljebine. Lower Aptian (Lenticularis Zone) of Greece (Viotía) Levadia, roadcut near Perachorion NW Levadia (BSPG 2003 XX 5807). Lower Aptian (Weissi - Furcata Zone) of Tanzania (Tanganyika, Mtwara) Nambawala plateau, Kikomolela, Likwaja; Kiturika Mts, Naiwanga, Mbate (MB K1431); Nambawala plateau, Kikomolela, Likwaja. Upper Aptian (Martinoides Zone) of Spain (Aragón, Teruel) Sección de Camarillas, camino del Bco. de la Canal; Maestrazgo, Barranco de las Corralizas (MGSB 78462); Sección de Camarillas, camino del Bco. de la Canal. Upper Aptian of Greece (Viotía) Aliartos, Chiarmena (BSPG 2003 XX 5412); Japan (Iwate-ken) Miyako-shi, Sakiyama, Hideshima (TUM 39742). Upper Aptian to Albian of Iran (Esfahan) Esfahan Basin, Dizlu. Earlymost Albian (Tardefurcata Zone) of Spain (Cataluña, Tarragona) Baix Penedès, Masarbones, field N (BSPG 2003 XX 6003). Lower Albian of Mexico (Baja California) Eréndira, Punto San Isidro (ERNO L-120404); Mexico (Sonora) Tuape, Cerro de la Espina. Middle Albian (Lautus Zone) of USA (Texas) Williamson County, west of Georgetown (TMM 1452TX2). Lower Cenomanian (Mantelli Zone) of Germany (Nordrhein/Westfalen) Mülheim/ Ruhr, Kassenberg. Lower Cenomanian (Dixoni Zone) of Germany (Sachsen) Meißen-Zscheila, Trinitatis church. Upper Cenomanian of Czech Republic (Central Bohemian region) Kolín, Planany (CGS HF 2661). Upper Cenomanian (Guerangeri Zone) of Czech Republic (Central Bohemian region) Korycany, Netreba (CGS HF 2660). Upper Cenomanian to Lower Turonian of Czech Republic (Ústí nad Labem region) Teplice (GPSL FLX 6315). Upper Cenomanian (Plenus Zone) of Germany (Sachsen) Dresden-Plauen. Uppermost Ceno- manian (Juddi Zone) of France (Aude) Les Corbières, Sougraigne, Prat-Périé (BSPG 2011 XXVI 4).

\section{Stelidioseris sp.}

Figure 8

Material. MGB 83270; 1 thin section.

Dimensions. See Table 4.

Remarks. The present specimen differs from known material by a septal symmetry with two cycles of each 11 septa. Stelidioseris mostly has a basic symmetry of six, eight or 10 . Uneven basic numbers are rare.

TABLE 4. Measurements of MGB 83270.

\begin{tabular}{ccccccc}
\hline & $\mathbf{n}$ & $\min -\mathbf{m a x}$ & $\boldsymbol{\mu}$ & $\mathbf{s}$ & $\mathbf{c v}$ & $\boldsymbol{\mu \pm \mathbf { s }}$ \\
\hline clmin & 20 & $0.92-1.30$ & 1.11 & 0.10 & 9.3 & $1.00-1.21$ \\
$\operatorname{clmax}$ & 20 & $1.09-1.61$ & 1.30 & 0.13 & 10.2 & $1.17-1.44$ \\
$\operatorname{ccd}$ & 30 & $1.20-1.88$ & 1.59 & 0.18 & 11.8 & $1.40-1.78$ \\
$\mathrm{~s}$ & 10 & $21-22$ & 21.80 & 0.42 & 1.9 & $21-22$ \\
\hline
\end{tabular}

Stelidioseris ? sp. Figure 9

Material. MGB 83366; 1 thin section.

Dimensions. See Table 5.

Remarks. The present material differs from Stelidioseris by the absence of a coenosteum; it is closer related to the genus Connectastrea Koby, 1905.

Superfamily AMPHIASTREOIDEA Ogilvie, 1897

Family AMPHIASTREIDAE Ogilvie, 1897

Genus AMPHIASTREA Etallon, 1859

Type species. Amphiastrea basaltiformis Etallon, 1859, by monotypy.

Amphiastrea basaltiformis Etallon, 1859

Figure 10 

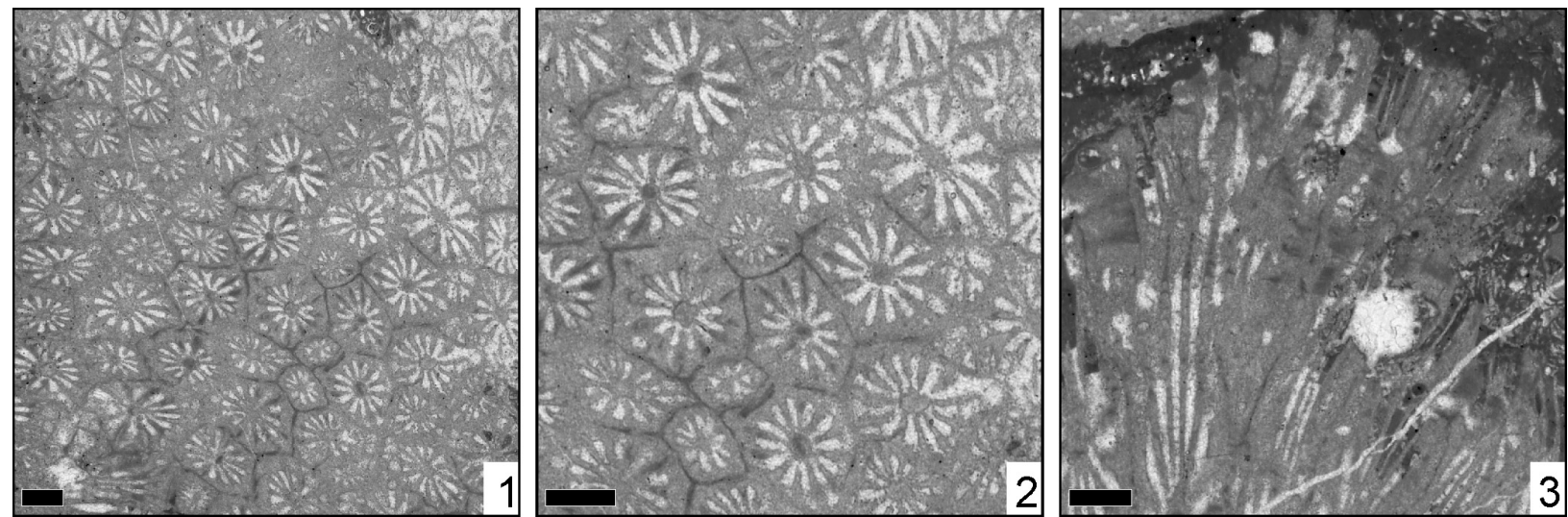

FIGURE 9. Stelidioseris ? sp., MGB 83366. 1: transversal thin section. 2: transversal thin section, detail. 3: longitudinal thin section. Scale $1 \mathrm{~mm}$.

TABLE 5. Measurements of MGB 83366.

\begin{tabular}{ccccccc}
\hline & $\mathbf{n}$ & $\mathbf{m i n}-\mathbf{m a x}$ & $\boldsymbol{\mu}$ & $\mathbf{s}$ & $\mathbf{c v}$ & $\boldsymbol{\mu \pm \mathbf { s }}$ \\
\hline clmin & 35 & $0.95-1.44$ & 1.22 & 0.12 & 10.1 & $1.10-1.35$ \\
$\operatorname{clmax}$ & 35 & $1.32-1.98$ & 1.66 & 0.17 & 10.2 & $1.49-1.83$ \\
$\operatorname{ccd}$ & 35 & $1.19-1.96$ & 1.53 & 0.20 & 13.2 & $1.32-1.73$ \\
$\mathrm{~s}$ & $6+6$ & & & & & \\
\hline
\end{tabular}

* 1859 Amphiastrea basaltiformis Etallon, p. 101

v 1888 Amphiastrea basaltiformis Koby; Koby, p. 433, pl. 115, fig. 1-3

1985 Amphiastraea basaltiformis Etallon, 1859; Geyer and Rosendahl, p. 167, pl. 2, fig. 5

v 2016 Amphiastrea basaltiformis Etallon, 1859; Löser, fig. A26ab

non 2018 Amphiastrea basaltiformis Etallon, 1859; Ricci, Lathuilière and Rusciadelli, p. 439, pl. 1, fig. 1 [= Amphiastrea sp.]

Material. MGB 83262; 1 thin section.

Dimensions. See Table 6.
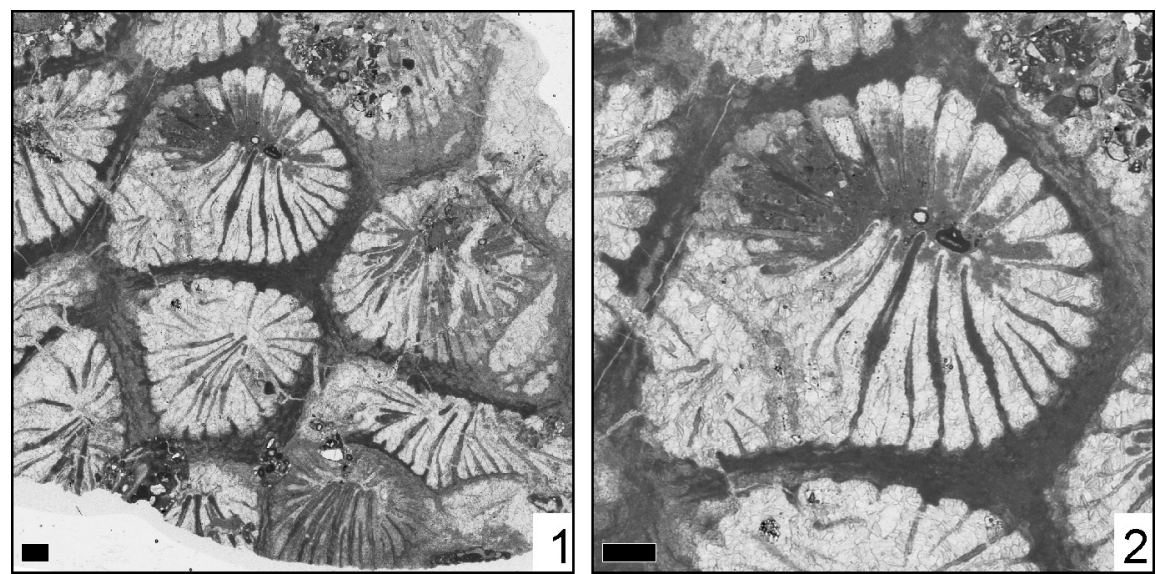

FIGURE 10. Amphiastrea basaltiformis Etallon, 1859, MGB 83262. 1: transversal thin section. 2: transversal thin section, detail. Scale $1 \mathrm{~mm}$. 

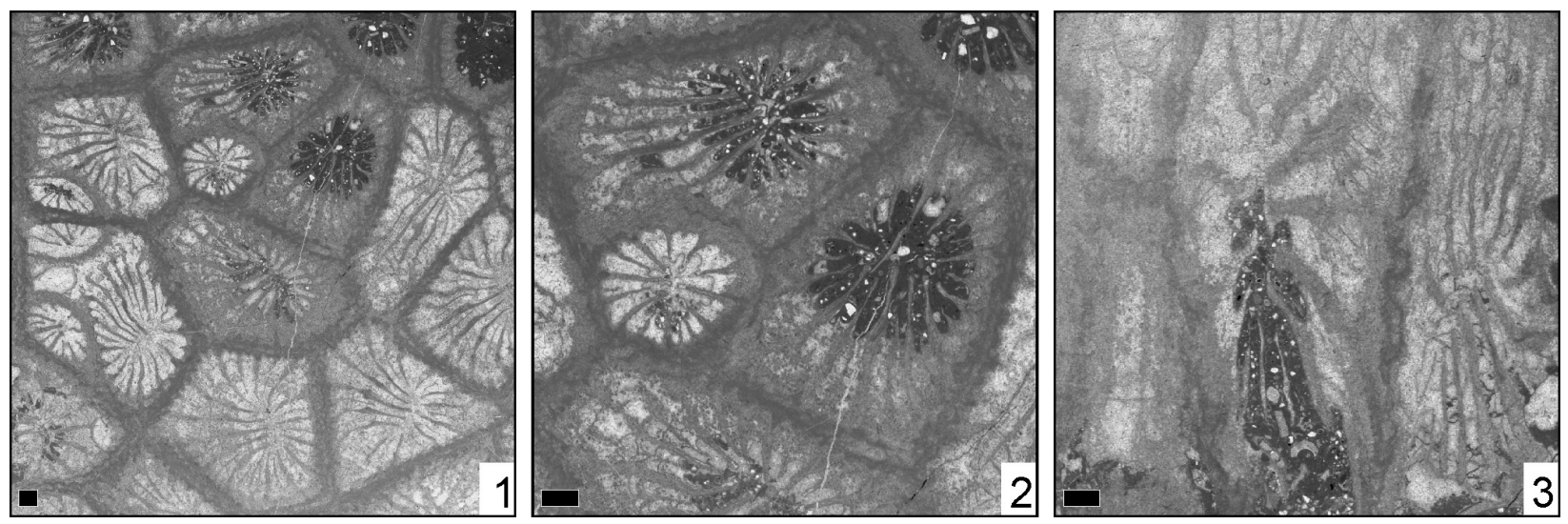

FIGURE 11. Amphiastrea cf. basaltiformis Etallon, 1859, MGB 83319. 1: transversal thin section. 2: transversal thin section, detail. 3: Oblique thin section. Scale $1 \mathrm{~mm}$.

Lower Berriasian of Czech Republic (Moravia) Štramberk (CGS HF 719). Lower Aptian of Poland (Malopolskie, Zakopane) Tatry Mts, Giewont (UJ $41 \mathrm{P} 1)$.

Amphiastrea cf. basaltiformis Etallon, 1859 Figure 11

Material. MGB 83319; 2 thin sections.

Dimensions. See Table 7.

Remarks. The present specimen is similar to $A$. basaltiformis but differs in smaller corallite dimensions.

TABLE 7. Measurements of MGB 83319.

\begin{tabular}{ccccccc}
\hline & $\mathbf{n}$ & $\min -\mathbf{m a x}$ & $\boldsymbol{\mu}$ & $\mathbf{s}$ & $\mathbf{c v}$ & $\boldsymbol{\mu \pm \mathbf { s }}$ \\
\hline clmin & 24 & $3.43-7.14$ & 5.09 & 1.00 & 19.6 & $4.08-6.09$ \\
clmax & 22 & $5.48-9.40$ & 7.23 & 0.93 & 12.8 & $6.30-8.17$ \\
ccd & 20 & $4.64-8.97$ & 6.41 & 1.07 & 16.7 & $5.34-7.49$ \\
$\mathrm{~s}$ & 17 & $23-37$ & 28.5 & 3.57 & 12.5 & $25-32$ \\
\hline
\end{tabular}

Amphiastrea cf. woodiae (Gregory, 1930)

Figure 12

Material. MGB 83274, 83334; 4 thin sections.

Dimensions. See Table 8.

Remarks. The material compares well to the type of $A$. woodiae, but has smaller corallite dimensions. The present material differs from the concept of the genus in having almost no septa in one face of the corallite, what is probably also the result of taphonomic processes.

TABLE 8. Measurements of MGB 83274.

\begin{tabular}{ccccccc}
\hline & $\mathbf{n}$ & $\min -\mathbf{m a x}$ & $\boldsymbol{\mu}$ & $\mathbf{s}$ & $\mathbf{c v}$ & $\boldsymbol{\mu \pm \mathbf { s }}$ \\
\hline clmin & 30 & $1.87-2.92$ & 2.31 & 0.24 & 10.4 & $2.07-2.56$ \\
clmax & 30 & $2.78-4.18$ & 3.35 & 0.36 & 10.9 & $2.98-3.71$ \\
ccd & 70 & $1.91-3.30$ & 2.61 & 0.33 & 12.7 & $2.28-2.95$ \\
$\mathrm{~s}$ & 20 & $11-23$ & 15.5 & 4.19 & 27.0 & $11-20$
\end{tabular}
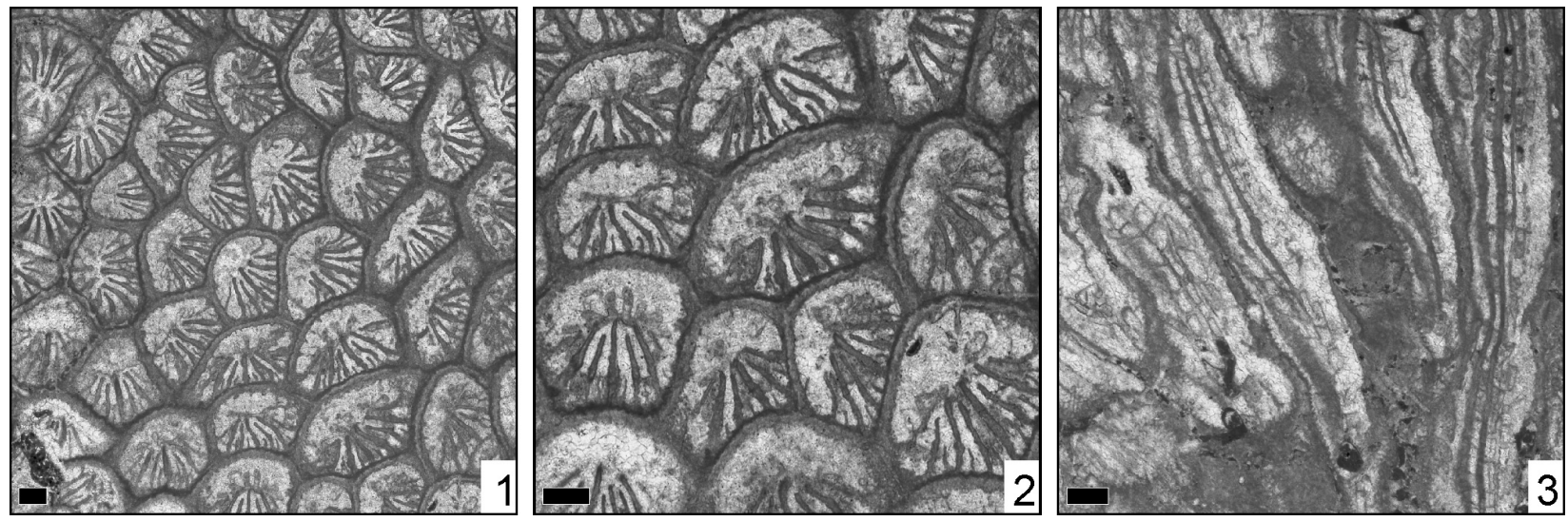

FIGURE 12. Amphiastrea cf. woodiae (Gregory, 1930), MGB 83274. 1: transversal thin section. 2: transversal thin section, detail. 3: longitudinal thin section. Scale $1 \mathrm{~mm}$. 

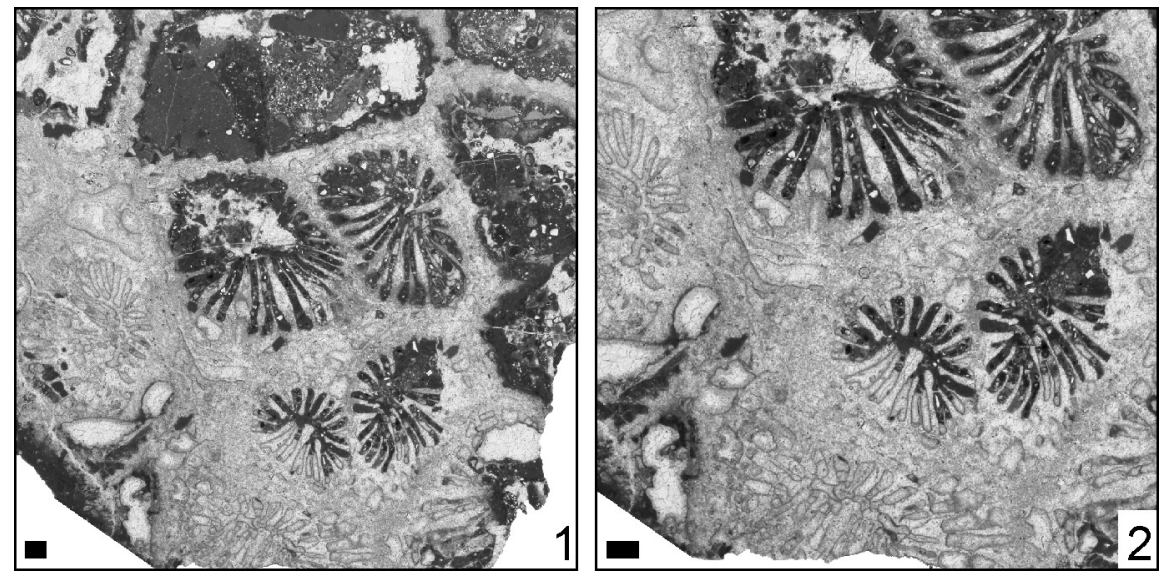

FIGURE 13. Amphiastrea sp., MGB 83249. 1: transversal thin section. 2: transversal thin section, detail. Scale $1 \mathrm{~mm}$.

\section{Amphiastrea sp.}

Figure 13

Material. MGB 83249; 1 thin section.

Dimensions. See Table 9.

Remarks. The only and very small specimen probably belongs to a new species. It could not be formally established because the small size of the coral that does not allow to prepare more than one thin section.

Occurrence. Tithonian to Lower Berriasian of Czech Republic (Moravia) Štramberk (CGS HF 90).

TABLE 9. Measurements of MGB 83249.

\begin{tabular}{ccccccc}
\hline & $\mathbf{n}$ & $\min -\mathbf{m a x}$ & $\boldsymbol{\mu}$ & $\mathbf{s}$ & $\mathbf{c v}$ & $\boldsymbol{\mu \pm \mathbf { s }}$ \\
\hline clmin & 6 & $3.34-4.91$ & 4.09 & 0.53 & 12.9 & $3.56-4.62$ \\
clmax & 6 & $5.82-8.36$ & 6.70 & 0.90 & 13.4 & $5.80-7.60$ \\
ccd & 5 & $4.28-7.62$ & 6.46 & 1.32 & 20.5 & $5.13-7.79$ \\
s & $25-28$ & & & & & \\
\hline
\end{tabular}

Family OPISTOPHYLLIDAE Geyer, 1955 Genus HYKELIPHYLLUM Eliášová, 1975

Type species. Hykeliphyllum lepidum Eliášová, 1975 , by original designation.

\section{Hykeliphyllum sp. \\ Figure 14}

Material. MGB 83261, 83263; 2 thin sections.

Dimensions. See Table 10.

Remarks. Hykeliphyllum was recently depicted and described (Löser, 2016). The genus is very similar to Amphiaulastrea Geyer, 1955, but differs by an absent or very narrow marginarium. The type species is the only formally described species;

TABLE 10. Measurements of MGB 83263.

\begin{tabular}{ccccccc}
\hline & $\mathbf{n}$ & $\mathbf{m i n}-\mathbf{m a x}$ & $\mathbf{\mu}$ & $\mathbf{s}$ & $\mathbf{c v}$ & $\boldsymbol{\mu \pm \mathbf { s }}$ \\
\hline clmin & 12 & $3.53-4.88$ & 4.06 & 0.51 & 12.5 & $3.55-4.57$ \\
clmax & 12 & $4.01-7.49$ & 5.91 & 1.24 & 20.9 & $4.67-7.15$ \\
$\mathrm{~s}$ & 7 & $21-28$ & 25.0 & 2.23 & 8.9 & $23-27$ \\
\hline
\end{tabular}
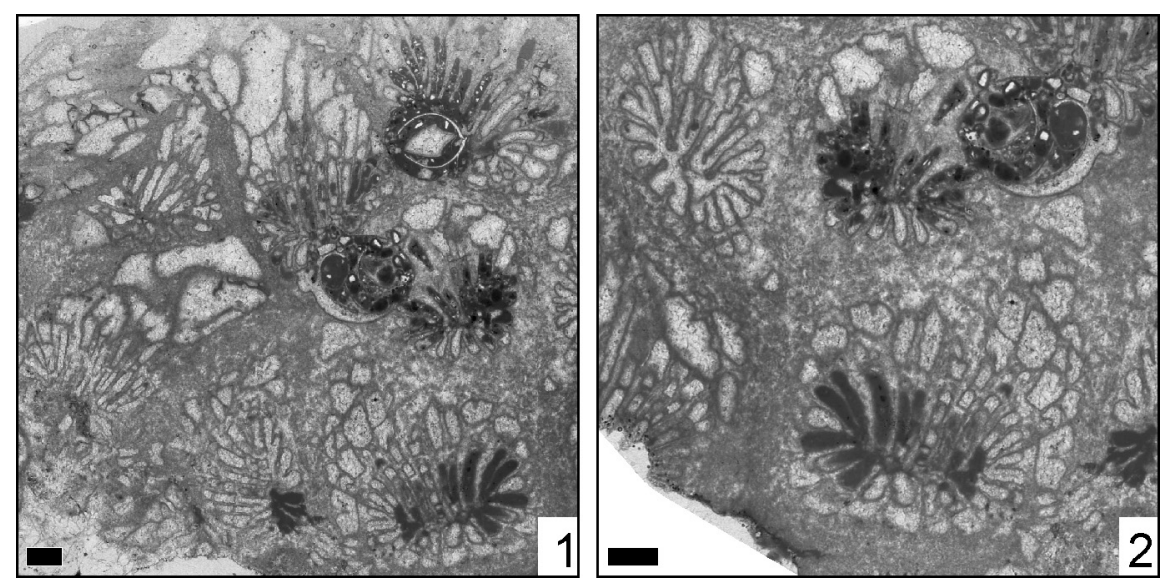

FIGURE 14. Hykeliphyllum sp., MGB 83261. 1: transversal thin section. 2: transversal thin section, detail. Scale $1 \mathrm{~mm}$. 

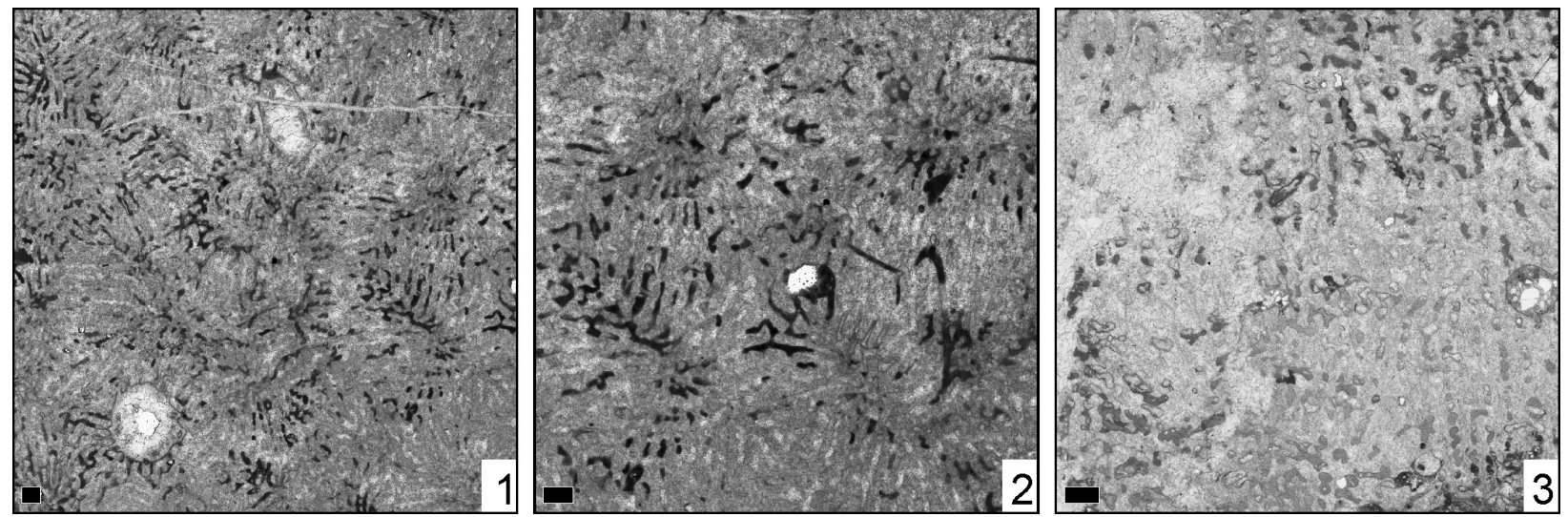

FIGURE 15. Astraeofungia diversisepta (Hackemesser, 1936), MGB 83350. 1: transversal thin section. 2: transversal thin section, detail. 3: Iongitudinal thin section. Scale $1 \mathrm{~mm}$.

Hykeliphyllum parvum Kuzmicheva, 2002 does not belong to this genus. The present material differs from the type species by smaller dimensions and a lower number of septa. It probably constitutes a new species, but the available material is not well enough preserved for it to be formally established.

Occurrence. Tithonian to Lower Berriasian of Czech Republic (Moravia) Štramberk (CGS HF 3238).

Superfamily CYCLOLITOIDEA Milne Edwards and Haime, 1849

Family LATOMEANDRIDAE Fromentel, 1861 Genus ASTRAEOFUNGIA Alloiteau, 1952

Type species. Astrea decipiens Michelin, 1841, by original designation.

Astraeofungia diversisepta (Hackemesser, 1936) Figure 15

v*1936 Thamnastraea diversisepta Hackemesser, p. 48 , pl. 6 , fig. 5

v 1996 Diploastrea harrisi Wells, 1932; BaronSzabo and Steuber, p. 25, pl. 14, figs. 2, 5

v 2003 Diploastrea harrisi Wells, 1932; BaronSzabo and González León, p. 212, figs. 8B, $\mathrm{E}$

v 2014 Astraeofungia stricta (Fromentel, 1857); Löser, p. 38, fig. $5 \mathrm{I}$

Material. MGB 83350; 2 thin sections.

Dimensions. See Table 11.

Remarks. The genus is well known and was recently described and depicted (Löser, 2016).

TABLE 11. Measurements of MGB 83350.

\begin{tabular}{ccccccc}
\hline & $\mathbf{n}$ & $\mathbf{m i n}-\mathbf{m a x}$ & $\boldsymbol{\mu}$ & $\mathbf{s}$ & $\mathbf{c v}$ & $\boldsymbol{\mu \pm \mathbf { s }}$ \\
\hline ccd & 20 & $4.74-8.45$ & 6.43 & 1.20 & 18.6 & $5.23-7.63$ \\
$\mathrm{~s}$ & 8 & $30-45$ & 36.5 & 5.65 & 15.4 & $31-42$ \\
\hline
\end{tabular}

Occurrence. Lower Kimmeridgian of Poland (Swietokrzyskie) Holy Cross Mts, Baltów (ZPAL Hiii1310). Cretaceous of Greece (Fokída) Kiona massif, Panourgias. Valanginian to Aptian of Mexico (Puebla) San Juan Raya (IGM 9248). Upper Barremian to Lower Aptian (Lenticularis Zone) of Mexico (Sonora) Ures, Cerro de Oro. Lower Aptian of Greece (Viotía) Arachova. Lower Albian of Mexico (Sonora) Ures, Cerro de Oro (ERNO L-4343). Lower Cenomanian (Dixoni Zone) of Spain (Cantabria, Santander) Cobreces, Luaña playa (BSPG 2007 V 087). Upper Cenomanian of Czech Republic (Central Bohemian region) Praha, Neratovice (OKSB nn). Upper Cenomanian (Plenus Zone) of Germany (Sachsen) Dresden-Plauen, Ratssteinbruch, southern quarry.

\section{Astraeofungia tenochi (Felix, 1891) Figure 16}

Material. MGB 83267; 2 thin sections.

v 1886 Synastraea maeandra; Fromentel, p. 598, pl. 173 , fig. 2 , pl. 175 , fig. 2

v*1891 Thamnastraea tenochi Felix, p. 145, pl. 22, figs. 7,7 a

v 1909a Thamnastraea Vaughani; Prever, p. 71, pl. 2, figs. 9,9 a

v 1951 Thamnasteria contorta Eguchi, n.sp.; Eguchi, p. 30 , pl. 5 , figs. 8,9 , pl. 6 , figs. 1,3

v 1951 Thamnasteria jezoensis Eguchi, n.sp.; Eguchi, p. 54, pl. 18, figs. 5, 6

v 1951 Thamnasteria contorta Eguchi, n.sp.; Eguchi, p. 30, pl. 5, figs. 8, 9, pl. 6, figs. 1, 3

v 1951 Thamnasteria jezoensis Eguchi, n.sp.; Eguchi, p. 54 , pl. 18 , figs. 5,6

v1963 Felixastraea mexicana n.sp.; Reyeros Navarro, p. 13, pl. 5, figs. 3, 4

v 1983 Thamnasteria crespoi (Felix, 1891); Reyeros de Castillo, p. 15, pl. 2, figs. 1, 2 

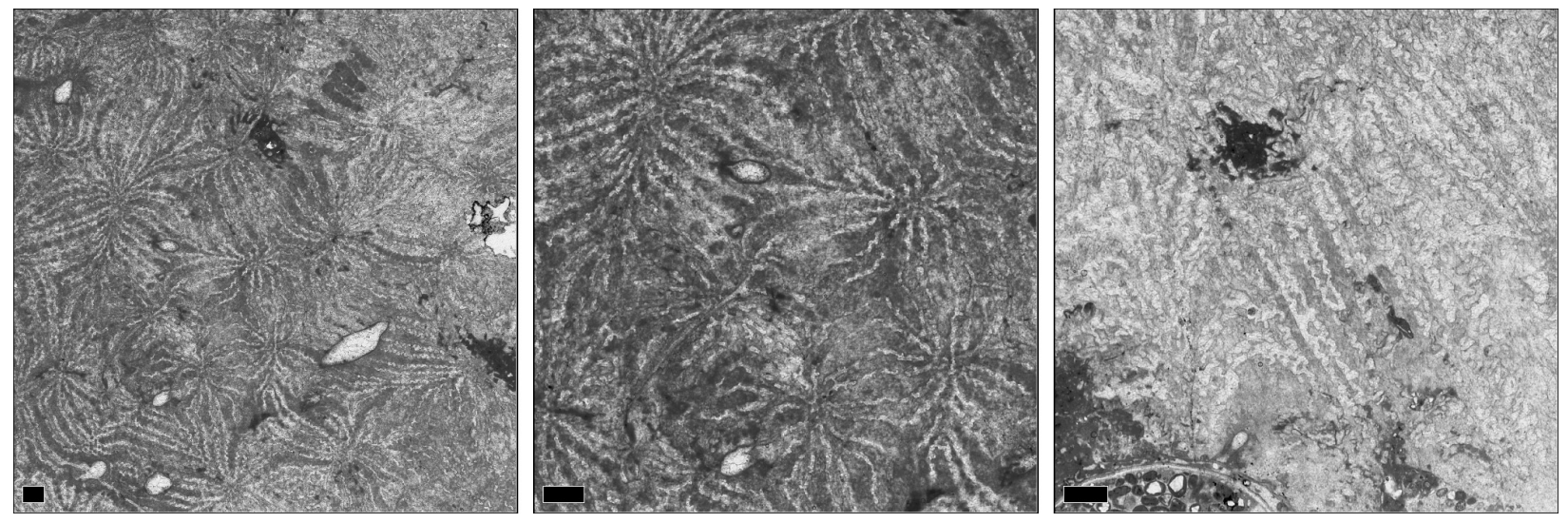

FIGURE 16. Astraeofungia tenochi Felix, 1891, MGB 83267. 1: transversal thin section. 2: transversal thin section, detail. 3: longitudinal thin section. Scale $1 \mathrm{~mm}$.

v 2003 Diploastrea harrisi Wells, 1932; BaronSzabo and González León, p. 212, figs. 8B, E

v 2006 Astraeofungia tenochi (Felix, 1891); Löser, p. 49 , fig. $3 \mathrm{~K}$

v 2013a Astraeofungia tenochi (Felix, 1891); Löser, fig. 3 , fig. 2

Dimensions. See Table 12.

TABLE 12. Measurements of MGB 83267.

\begin{tabular}{ccccccc}
\hline & $\mathbf{n}$ & $\min -\mathbf{m a x}$ & $\boldsymbol{\mu}$ & $\mathbf{s}$ & $\mathbf{c v}$ & $\boldsymbol{\mu \pm \mathbf { s }}$ \\
\hline $\mathrm{ccd}$ & 20 & $3.52-6.85$ & 5.11 & 1.04 & 20.5 & $4.06-6.16$ \\
$\mathrm{~s}$ & 10 & $22-31$ & 25.3 & 3.05 & 12.0 & $22-28$ \\
\hline
\end{tabular}

Remarks. The present specimen has slightly larger dimensions than the holotype of Astraeofungia tenochi.

Occurrence. Valanginian to Aptian of Mexico (Puebla) San Juan Raya. Lower Hauterivian (Radiatus Zone) of France (Yonne) Gy-l'Evêque (MHNG 4560); Leugny (MNHN); Gy-l'Evêque; Fontenoy, field $S$ the junction to Les Merles; Gyl'Evêque; Fontenoy, field $S$ the junction to Les Merles. Barremian of Mexico (Puebla) Tehuacán, San Antonio Texcala. Upper Barremian of France (Ardèche) St.Remèze, Pont de Laval (BSPG 2003 XX 5247). Upper Barremian to Lower Aptian (Lenticularis Zone) of Mexico (Sonora) Ures, Cerro de Oro (ERNO L-4330). Lower Aptian of Italy (Abruzzi, L'Aquila) Monti d'Ocre, Fossa Cerasetti. Lower Upper Aptian of Algeria (Tebessa) Commune Ouenza, Ouenza Mt (UP M 5139); Spain (Cataluña, Lérida) La Noguera, Montsec de Rubies, section NW La Cabrua quarry (BSPG 2003 XX 6326). Upper Aptian of Japan (Hokkai-do) Asibetsu-shi, Shimonoshita tunnel. Latest Aptian of Japan (Iwate-ken) Shimohei-gun, Tanohata-mura, Haipe, northern cliff. Earlymost Albian (Tardefurcata Zone) of Spain (Cataluña, Barcelona) Alt Penedès, Castellvi de la Marca, Can Pascual, section loc. 2 (BSPG 2003 XX 6282); Spain (Cataluña, Tarragona) Baix Penedès, Masarbones, field N (ERNO L6034). Lower Albian of Mexico (Oaxaca) Tepelmeme, El Rodeo Ranch; Mexico (Sonora) Ures, Cerro de Oro (ERNO L-4340). Middle Albian of Mexico (Sonora) Tepache, Lampazos area, Espinazo de Diablo (ERNO L-120526). Lower Cenomanian of Greece (Kozani) Kozani, Nea Nikopoli (BSPG 2003 XX 5820). Lower Cenomanian (Dixoni Zone) of Spain (Cantabria, Santander) Cobreces, Luaña playa (BSPG 2007 V 012). Middle to Upper Cenomanian (Rhotomagense - Naviculare Zone) of France (Sarthe) Le Mans. Upper Cenomanian of Czech Republic (Central Bohemian region) Kolín, Planany (OKSB n/a (L7572)). Upper Cenomanian (Plenus Zone) of Germany (Sachsen) Dresden-Plauen, Ratssteinbruch, southern quarry (BSPG 2009 XVII 52). Latest Cenomanian (Juddi Zone) of France (Aude) Les Corbières, Sougraigne, Prat-Périé (BSPG 2011 XXVI 3). Lower Turonian (Nodosoides Zone) of Portugal (Coimbra) Nazaré, beach section (ERNO L-132504).

Genus LATIASTREA Beauvais, 1964

Type species. Latiastrea foulassensis Beauvais, 1964 , by original designation.

Latiastrea canavarii (Prever, 1909)

Figure 17

v*1909a Latimaeandraraea Canavarii Prever, p. 100, pl. 9, fig. 4

v 1909a Latimaeandraraea Douvilléi; Prever, p. 102, pl. 7, fig. 5, pl. 9, fig. 7

v 1955 Microphyllia bachmayeri; Geyer, p. 205, pl. 23, fig. 5 pl. 25 , fig. 2 

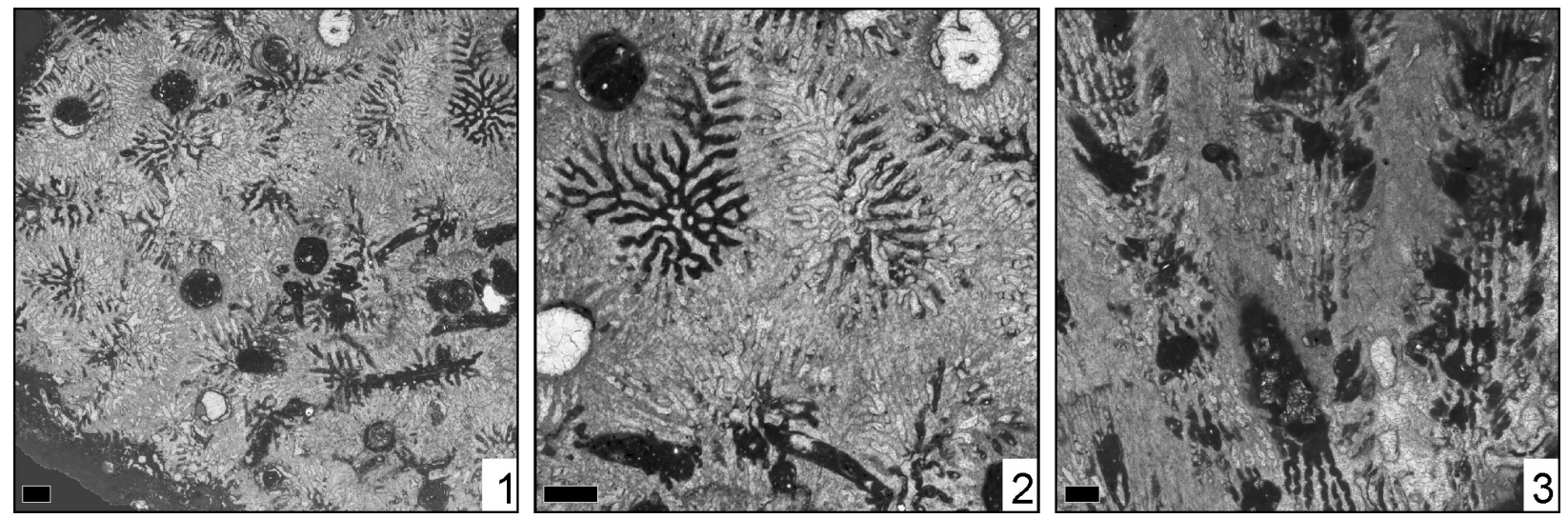

FIGURE 17. Latiastrea canavarii (Prever, 1909), MGB 83311. 1: transversal thin section. 2: transversal thin section, detail. 3: longitudinal thin section. Scale $1 \mathrm{~mm}$.

TABLE 13. Measurements of MGB 83311.

\begin{tabular}{ccccccc}
\hline & $\mathbf{n}$ & $\mathbf{m i n}-\mathbf{m a x}$ & $\boldsymbol{\mu}$ & $\mathbf{s}$ & $\mathbf{c v}$ & $\boldsymbol{\mu \pm \mathbf { s }}$ \\
\hline $\operatorname{clmin}$ & 15 & $1.90-2.69$ & 2.39 & 0.26 & 11.0 & $2.13-2.66$ \\
$\operatorname{clmax}$ & 15 & $3.50-5.71$ & 4.37 & 0.63 & 14.4 & $3.74-5.00$ \\
$\operatorname{ccd}$ & 20 & $2.83-4.56$ & 3.66 & 0.55 & 15.0 & $3.11-4.21$ \\
$\mathrm{~s}$ & 8 & $32-41$ & 37.6 & 3.85 & 10.2 & $34-41$
\end{tabular}

Material. MGB 83305, 83311, 83322, 83344, 83349; 2 thin sections.

Dimensions. See Table 13.

Remarks. Latiastrea has a transitional position between Thalamocaeniopsis and monoserial Microphyllia. The genus was recently depicted and described (Löser, 2016).

Occurrence. Kimmeridgian of France (Jura) Valfin (MNHN R10749). Tithonian to Lower Berriasian of Czech Republic (Moravia) Štramberk. Lower Aptian of Italy (Abruzzi, L'Aquila) Monti d'Ocre, Fossa Cerasetti.

\section{Latiastrea somaensis (Eguchi, 1951)}

Figure 18

v*1951 Latomeandra somaensis Eguchi, p. 78, pl. 20, fig. 6

v 1951 Latomeandra tosaensis Eguchi, n. sp.; Eguchi, p. 66 , pl. 24 , fig. 7 , pl. 25 , fig. 2

?1985 Microphyllia undans Etallon, 1859; Geyer and Rosendahl, p. 167, pl. 2, fig. 4

v2002 Latomeandra somaensis (Eguchi); Löser and Mori, p. 102

Material. MGB 83241, 83244, 83287, 83317, 83324; 5 thin sections.

Dimensions. See Table 14.

Occurrence. Doggerian to Malmian of Japan (Fukushima-ken) Soma-shi, Yawata, Tomizawa. Callovian to Kimmeridgian of Japan (Kochi-ken) Takaoka-gun, Sakawa-cho, Kamo, Mitoda.

\section{Latiastrea sp.}

Figure 19

v1897 Isastraea gourdani Fromentel; Ogilvie, p. 192, pl. 15, fig. 17
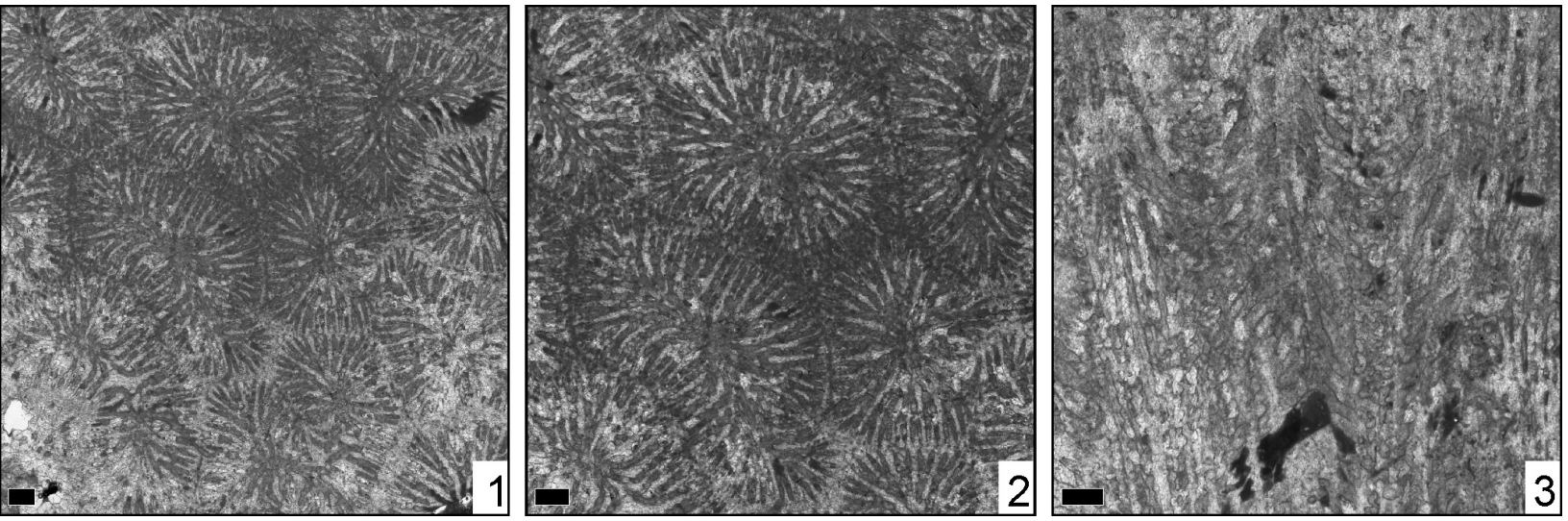

FIGURE 18. Latiastrea somaensis Eguchi, 1951, MGB 83244. 4: transversal thin section. 5: transversal thin section, detail. 6: longitudinal thin section. Scale $1 \mathrm{~mm}$. 
TABLE 14. Measurements of MGB 83244.

\begin{tabular}{ccccccc}
\hline & $\mathbf{n}$ & $\min -\mathbf{m a x}$ & $\boldsymbol{\mu}$ & $\mathbf{s}$ & $\mathbf{c v}$ & $\boldsymbol{\mu \pm \mathbf { s }}$ \\
\hline clmin & 20 & $3.26-5.28$ & 4.23 & 0.54 & 12.8 & $3.68-4.77$ \\
clmax & 20 & $4.96-8.68$ & 6.51 & 1.14 & 17.5 & $5.37-7.65$ \\
ccd & 20 & $4.59-6.42$ & 5.41 & 0.59 & 11.0 & $4.81-6.01$ \\
$\mathrm{~s}$ & 7 & $53-73$ & 62.4 & 8.65 & 13.8 & $54-71$ \\
\hline
\end{tabular}

Material. MGB 83356, 83360, 83387; 1 thin section.

Dimensions. See Table 15.

Occurrence. Callovian to Kimmeridgian of Japan (Kochi-ken) Takaoka-gun, Sakawa-cho, Kamo, Mitoda (TUM 65338). Tithonian to Lower Berriasian of Czech Republic (Moravia) Štramberk.

Genus LATOMEANDRA Milne Edwards and Haime, 1849

Type species. Lithodendron plicata Goldfuss, 1826 , by subsequente definition.

Latomeandra isseli (Prever, 1909)

Figure 20

v*1909a Diplarea isseli Prever, p. 1000, figs. 15-17

Material. MGB 83232, 83332; 2 thin sections.

Dimensions. See Table 16.

Remarks. Latomeandra is a problematic genus because the type material of the type species is silicified. Thin sections have never been prepared. The present material compares well to the type species in its outer appearance. The corallites have an irregular outline and are densely arranged.

Occurrence. Tithonian of Italy (Abruzzi, L'Aquila) Gran Sasso, Calascio. Lower Hauterivian (Radiatus Zone) of France (Yonne) Gy-l'Evêque (FSL nn); Fontenoy, field $S$ the junction to Les Merles (BSPG 2003 XX 5425).

Genus OVALASTREA Orbigny, 1849
TABLE 15. Measurements of MGB 83387.

\begin{tabular}{ccccccc}
\hline & $\mathbf{n}$ & $\min -\max$ & $\boldsymbol{\mu}$ & $\mathbf{s}$ & $\mathbf{c v}$ & $\boldsymbol{\mu} \pm \mathbf{s}$ \\
\hline clmin & 11 & $2.78-4.37$ & 3.77 & 0.44 & 11.7 & $3.33-4.21$ \\
clmax & $4-5.8$ & & & & & \\
$\mathrm{~s}$ & 5 & $46-57$ & 49.0 & 4.52 & 9.2 & $44-54$ \\
\hline
\end{tabular}

Type species. Astrea caryophylloides Goldfuss, 1826, by monotypy.

Ovalastrea caryophylloides (Goldfuss, 1826)

Figure 21

v*1826 Astrea caryophylloides Goldfuss, p. 66, pl. 22, fig. 7

v 1857 Favia hemisphaerica; Fromentel, p. 35, pl. 4, fig. 7

v 1879 Favia hemisphaerica; Fromentel, p. 481, pl. 118, fig. 2

v 1887 Cyathoseris facilis; Pocta, p. 36, pl. 2, fig. 2

v 1896 Favia Schmidti; Koby, p. 49, pl. 6, fig. 2

v1925 Favia bihinense; Gregory, p. 23, pl. 4, fig. 1

v 1963 Baryphyllia confusa (d'Orbigny); Reyeros Navarro, p. 12, pl. 3, figs. 1, 2

v 1963 Complexastrea cyclops (Felix); Reyeros Navarro, p. 16, pl. 5, figs. 1, 2, 5

v1964 Ellipsocoenia hemispherica (de From., 1857); Morycowa, p. 97, pl. 32, fig. 1, pl. 33, fig. 1

v $2001 \quad$ Chorisastraea sp.; Löser, p. 45, pl. 3, fig. 1

v 2016 Astrea caryophylloides Goldfuss, 1826; Löser, fig. 013

Material. MGB 83236; 2 thin sections.

Dimensions. See Table 17.

Remarks. The genera was recently provided with a modern description (Löser, 2016), and the type of the type species was depicted.

Occurrence. Bathonian of Somalia, Bihendula. Kimmeridgian (Beckeri Zone, Ulmense Subzone)
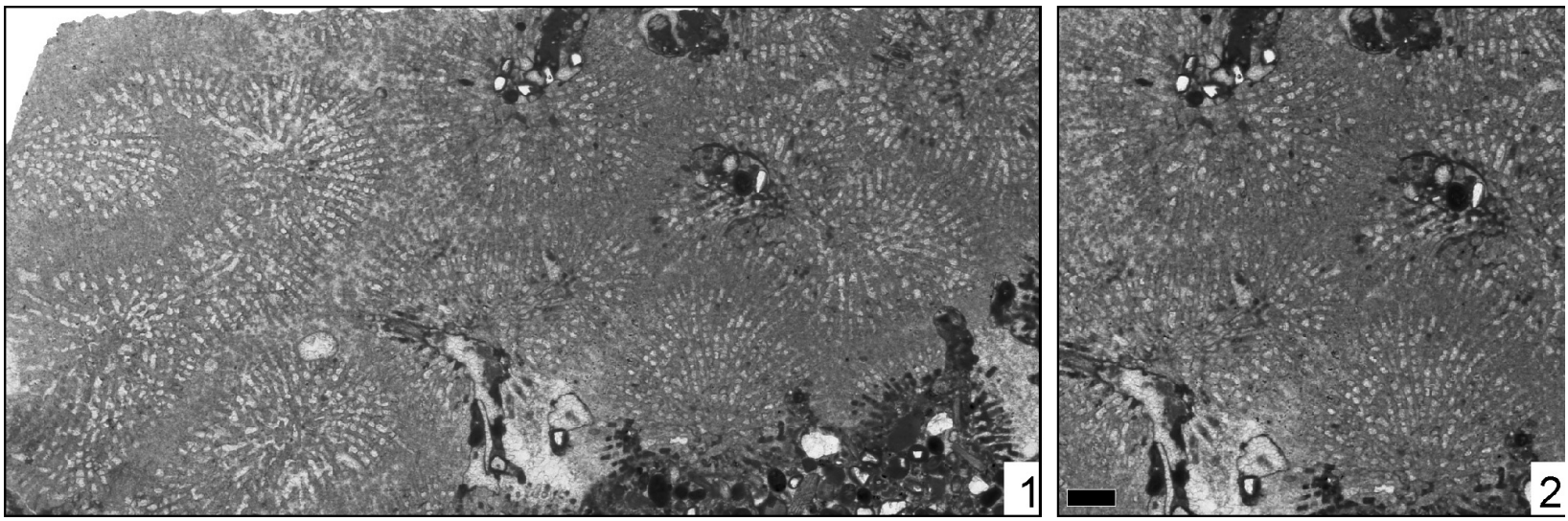

FIGURE 19. Latiastrea sp., MGB 83387. 1: transversal thin section. 2: transversal thin section, detail. Scale $1 \mathrm{~mm}$. 

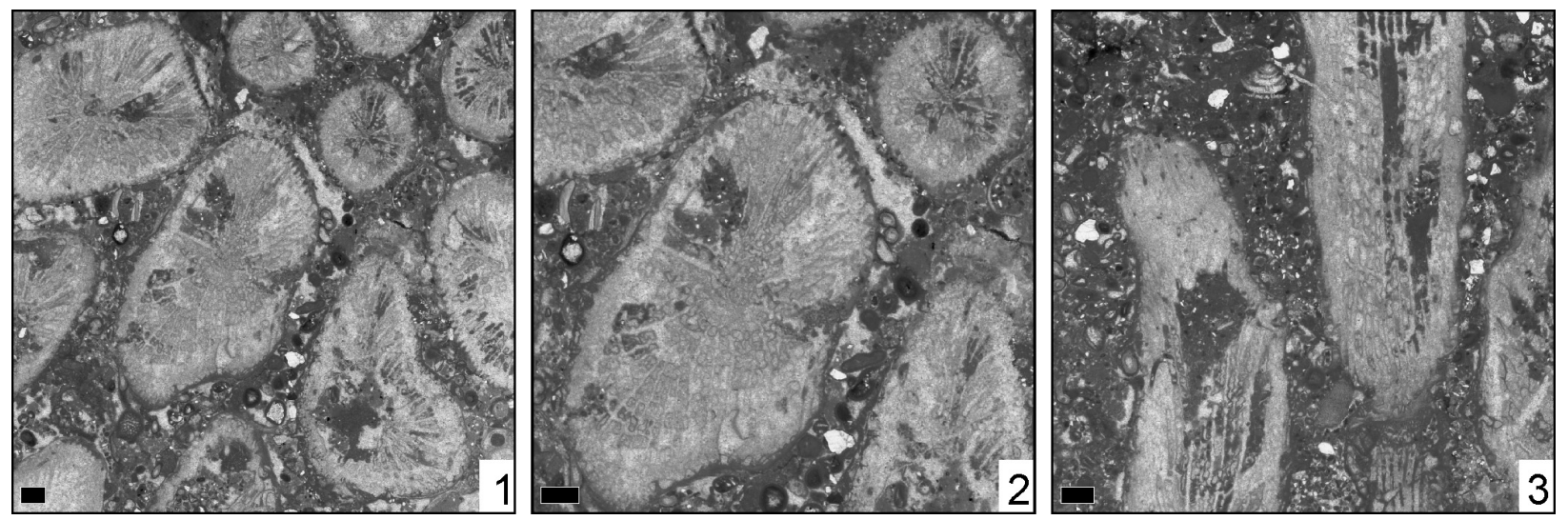

FIGURE 20. Latomeandra isseli (Prever, 1909), MGB 83232. 1: transversal thin section. 2: transversal thin section, detail. 3: longitudinal thin section. Scale $1 \mathrm{~mm}$.

TABLE 16. Measurements of MGB 83232.

\begin{tabular}{ccccccc}
\hline & $\mathbf{n}$ & min-max & $\boldsymbol{\mu}$ & $\mathbf{s}$ & $\mathbf{c v}$ & $\boldsymbol{\mu \pm \mathbf { s }}$ \\
\hline cmax & 10 & $4.06-11.2$ & 7.34 & 2.24 & 30.4 & $5.10-9.58$ \\
cmin & 10 & $3.35-7.08$ & 4.94 & 1.15 & 23.3 & $3.79-6.10$ \\
$\mathrm{~s}$ & $70-80$ & & & & & \\
\hline
\end{tabular}

of Germany (Baden-Württemberg) Stuttgart, Giengen an der Brenz. Valanginian to Aptian of Mexico (Puebla) San Juan Raya. Lower Hauterivian (Radiatus Zone) of France (Yonne) Gy-l'Evêque; Fontenoy, field $S$ the junction to Les Merles (BSPG 2003 XX 5443); Gy-l'Evêque; Fontenoy, field S the junction to Les Merles. Upper Barremian to Lower Aptian (Sartousi - Weissi Zone) of Switzerland (Schwyz) Drusberg, Käsernalp. Aptian of Spain (Aragón, Teruel) Sierra de Albarracín, between Moscardon and Royuela (ERNO L-6845). Lower Aptian of Poland (Malopolskie, Wadowice) Lanckorona, Jastrzebia. Lower Cenomanian of France (Charente-Maritime) Fouras (BSPG 2003 XX
TABLE 17. Measurements of MGB 83236.

\begin{tabular}{ccccccc}
\hline & $\mathbf{n}$ & min-max & $\boldsymbol{\mu}$ & $\mathbf{s}$ & $\mathbf{c v}$ & $\boldsymbol{\mu \pm \mathbf { s }}$ \\
\hline ccd & 7 & $8.46-14.5$ & 11.9 & 2.22 & 18.7 & $9.65-14.1$ \\
clmin & 5 & $5.85-6.68$ & 6.26 & 0.37 & 5.9 & $5.89-6.64$ \\
clmax & 5 & $10.3-11.8$ & 11.1 & 0.68 & 6.1 & $10.5-11.8$ \\
s & $60-70$ & & & & & \\
\hline
\end{tabular}

5595). Upper Cenomanian (Guerangeri Zone) of Czech Republic (Central Bohemian region) Korycany.

\section{Genus PERISERIS Ferry, 1870}

Type species. Agaricia elegantula Orbigny, 1850, by monotypy.

Periseris crassisepta (Sikharulidze, 1985)

Figure 22

* 1985 Microsolena crassisepta Sikharulidze, p. 49, pl. 22, fig. 4
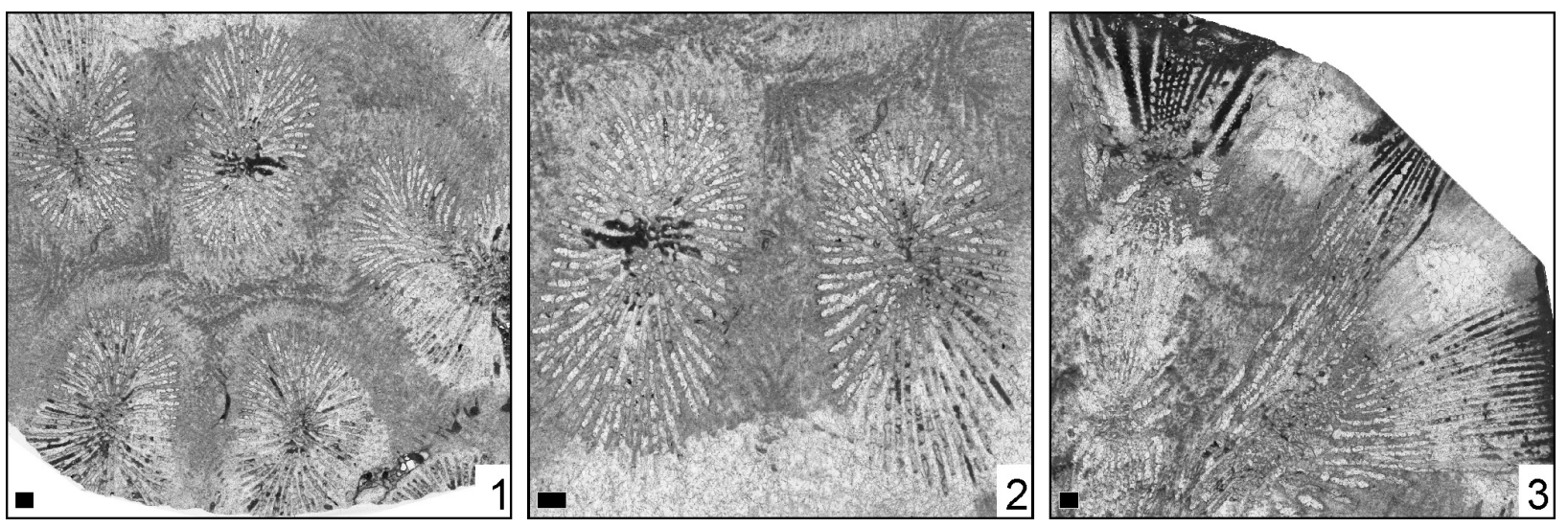

FIGURE 21. Ovalastrea caryophylloides Goldfuss, 1826, MGB 83236. 1: transversal thin section. 2: transversal thin section, detail. 3: longitudinal thin section. Scale $1 \mathrm{~mm}$. 

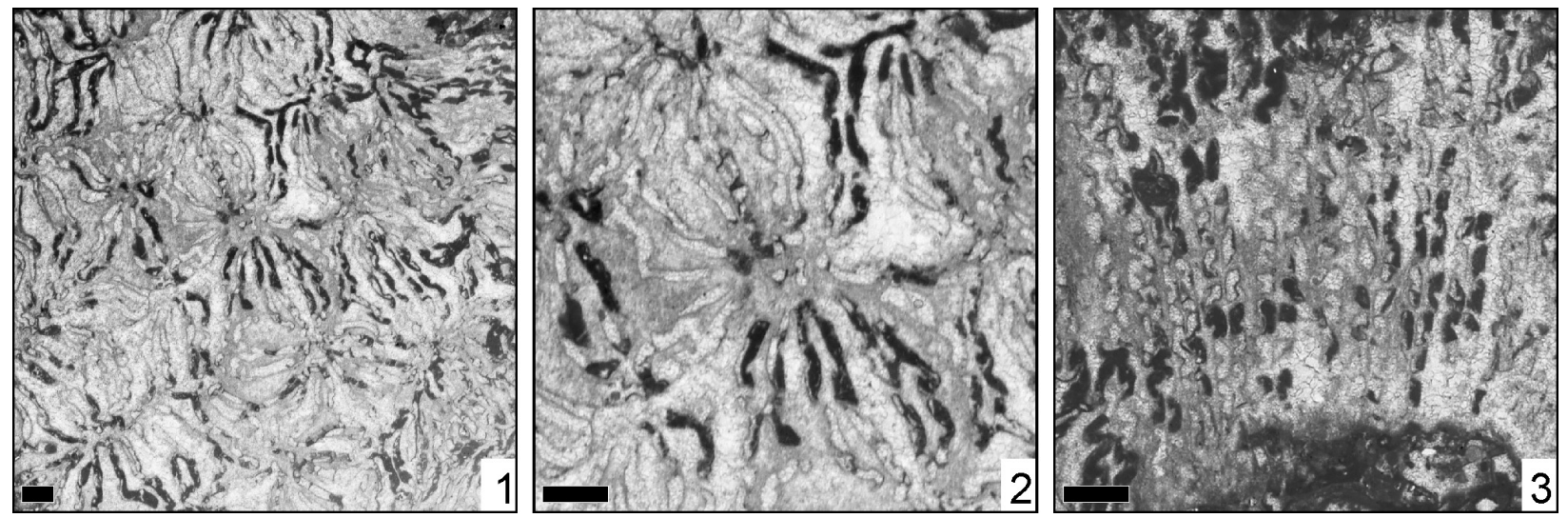

FIGURE 22. Periseris crassisepta Sikharulidze, 1985, MGB 83268. 1: transversal thin section. 2: transversal thin section, detail. 3: Iongitudinal thin section. Scale $1 \mathrm{~mm}$.

v $2001 \quad$ Periseris sp.; Löser, p. 46, pl. 3, fig. 3

Material. MGB 83268; 3 thin sections.

Dimensions. See Table 18.

Remarks. The present specimen compares well to the description and illustration given by Sikharulidze.

Occurrence. Kimmeridgian of Germany (Bayern) Saal an der Donau (FLH 3701). Upper Valanginian of Spain (Murcia) Sierra Larga (MGB 78397). Hauterivian of Georgia (Imereti) Godogani. Lower Hauterivian (Radiatus Zone) of France (Yonne) Fontenoy, field $S$ the junction to Les Merles (BSPG 2003 XX 5263); Fontenoy, field $N$ the junction to

TABLE 18. Measurements of MGB 83268.

\begin{tabular}{ccccccc}
\hline & $\mathbf{n}$ & $\mathbf{m i n}-\mathbf{m a x}$ & $\boldsymbol{\mu}$ & $\mathbf{s}$ & $\mathbf{c v}$ & $\boldsymbol{\mu \pm \mathbf { s }}$ \\
\hline $\mathrm{crd}$ & 6 & $3.51-4.40$ & 3.92 & 0.34 & 8.6 & $3.58-4.26$ \\
$\mathrm{cdw}$ & 6 & $3.09-5.52$ & 4.22 & 0.90 & 21.5 & $3.31-5.12$ \\
$\mathrm{~s}$ & 10 & $13-20$ & 17.2 & 2.48 & 14.4 & $15-20$ \\
\hline
\end{tabular}

Les Merles (BSPG 2003 XX 5072); Leugny, Les Cassines 4 km E Leugny (BSPG 2003 XX 6050).

Periseris elegantula (Orbigny, 1850)

Figure 23

V*1850 Agaricia elegantula Orbigny, p. 293

1990 Periseris elegantula (d'Orbigny, 1850); Lathuilière, p. 38, pl. 1, figs. 1-2, pl. 2, figs. $1-4$, pl. 3 , figs. $1-6$, pl. 4 figs. $1-7$, pl. 5 figs. 1-6

v 2006 Microsolena sp.; Löser and Ferry, p. 484, figs. 5.7-9

v 2013a Periseris ? crassisepta (Sikharulidze, 1985); Löser, fig. 3, fig. 5

v 2016 Agaricia elegantula Orbigny, 1850; Löser, figs. P43

Material. MGB 83277; 2 thin sections.

Dimensions. See Table 19.

Remarks. The genus and species were recently described and depicted (Löser, 2016). The present material coincides well with the type material of Periseris elegantula. The distance of the corallite
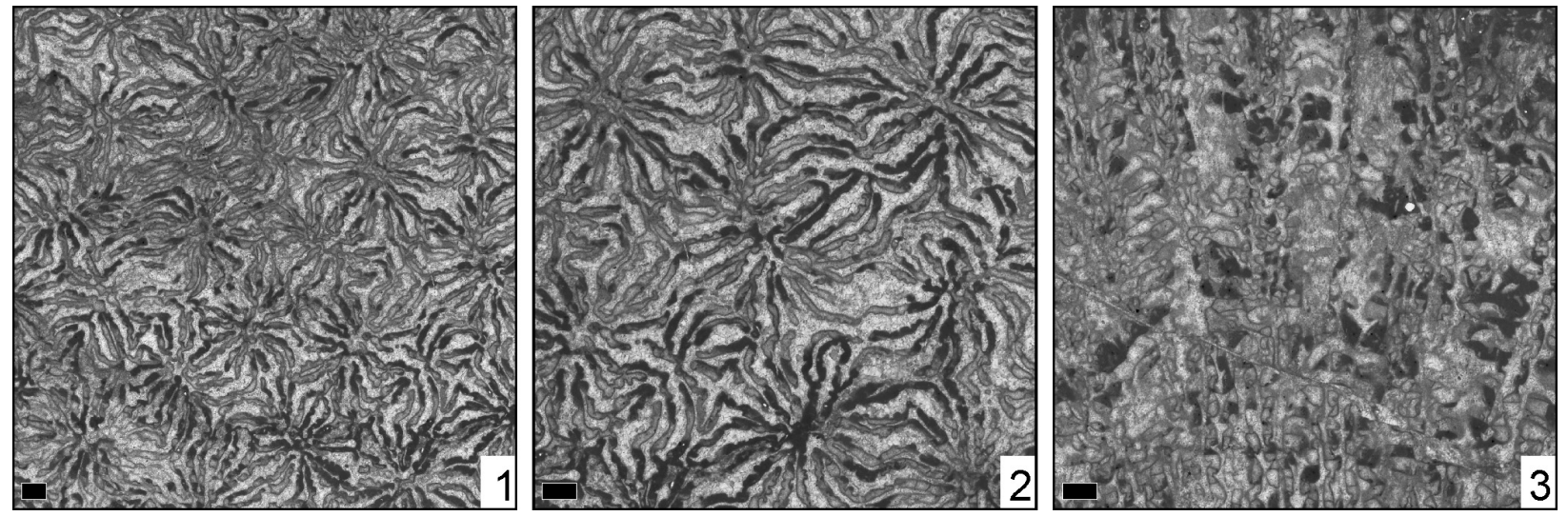

FIGURE 23. Periseris elegantula Orbigny, 1850, MGB 83277. 1: transversal thin section. 2: transversal thin section, detail. 3: longitudinal thin section. Scale $1 \mathrm{~mm}$. 
TABLE 19. Measurements of MGB 83277.

\begin{tabular}{ccccccc}
\hline & $\mathbf{n}$ & min-max & $\boldsymbol{\mu}$ & $\mathbf{s}$ & $\mathbf{c v}$ & $\boldsymbol{\mu \pm \mathbf { s }}$ \\
\hline ccd & 25 & $3.04-5.62$ & 4.44 & 0.73 & 16.5 & $3.71-5.18$ \\
crd & 15 & $3.26-4.72$ & 3.96 & 0.40 & 10.3 & $3.55-4.37$ \\
$\mathrm{~s}$ & 20 & $18-28$ & 21.9 & 3.12 & 14.2 & $19-25$ \\
\hline
\end{tabular}

rows and the distance of the corallites within the rows are slightly larger in the present material.

Occurrence. Bajocian of France (Haute-Marne) Langres (MNHN A26574). Kimmeridgian of Germany (Bayern) Saal an der Donau (FLH 3266). Tithonian of Japan (Fukushima-ken) Soma-gun area (TUM nn). Lower Hauterivian (Radiatus Zone) of France (Yonne) Fontenoy, field $S$ the junction to Les Merles (BSPG 2003 XX 5290); Fontenoy, field $\mathrm{N}$ the junction to Les Merles (BSPG $2003 \mathrm{XX}$ 5070); Fontenoy, section in Guillorets W Fontenoy (BSPG 2003 XX 5054); Leugny, Les Cassines 4 km E Leugny; Gy-l'Evêque (MNHN nn); Leugny, Les Cassines $4 \mathrm{~km} E$ Leugny. Upper Hauterivian of Japan (Miyagi-ken) Kesennuma-shi, Oshima, Yogai (TUM 65384). Upper Barremian of France (Ardèche) St.Remèze, Pont de Laval.

Periseris frondescens (Orbigny, 1850)

Figure 24

v*1850 Synastrea frondescens Orbigny, (2), p. 94

v 1887 Thamnastrea renevieri; Koby, p. 379, pl. 103, fig. 2

v 1936 Centrastraea ex. aff. insignis de Fromentel 1887; Hackemesser, p. 56

v 2006 Microsolena sp.; Löser and Ferry, p. 484, figs. 5.7-9

Material. MGB 83323; 2 thin sections.

Dimensions. See Table 20.
TABLE 20. Measurements of MGB 83323.

\begin{tabular}{ccccccc}
\hline & $\mathbf{n}$ & $\mathbf{m i n}-\mathbf{m a x}$ & $\boldsymbol{\mu}$ & $\mathbf{s}$ & $\mathbf{c v}$ & $\boldsymbol{\mu \pm \mathbf { s }}$ \\
\hline $\mathrm{crd}$ & 10 & $2.45-3.22$ & 2.77 & 0.24 & 8.9 & $2.52-3.02$ \\
$\mathrm{cdw}$ & 10 & $1.41-3.24$ & 2.22 & 0.66 & 29.6 & $1.56-2.89$ \\
$\mathrm{~s}$ & 10 & $19-32$ & 25.7 & 3.97 & 15.4 & $22-30$ \\
\hline
\end{tabular}

Remarks. The present specimen differs from the type material of Periseris frondescens by less regular calicular rows.

Occurrence. Bajocian of Germany (Bayern) Amberg, Bernricht (ABP K2). Callovian of Switzerland (Vaud) Jura, Ste. Croix. Kimmeridgian of Germany (Bayern) Saal an der Donau (FLH 3749). Cretaceous of Greece (Fokída) Kiona massif, Panourgias. Lower Hauterivian (Radiatus Zone) of France (Yonne) Saint-Sauveur. Upper Barremian of France (Ardèche) St. Remèze, Pont de Laval.

Genus PLACOSERIS Fromentel, 1863

Type species. Placoseris patella Fromentel, 1863, by subsequente definition.

Placoseris poculum (Fromentel, 1857)

Figure 25

v*1857 Trochoseris poculum Fromentel, p. 18, pl. 1, figs. 5,6

v 1863b Leptophyllia poculum; Fromentel, p. 304, pl. 48, fig. 1

vp 1989 Acrosmilia patellata (Michelin 1845); Löser, p. 131 , text-fig. 34 , pl. 26, fig. 1

vp 1994 ? Acrosmilia sp.; Löser, p. 30, text-fig. 1921, pl. 10, fig. 6

v 2014 Acrosmilia patella (de Fromentel, 1863); Löser, p. 35, fig. $5 f$

Material. MGB 83260; 1 thin section.

Dimensions. (83260) c, $2.3 \times 16.1 \mathrm{~mm}$; s, 143 .

Remarks. In the previous literature, the genus Placoseris was considered synonymous with Acros-
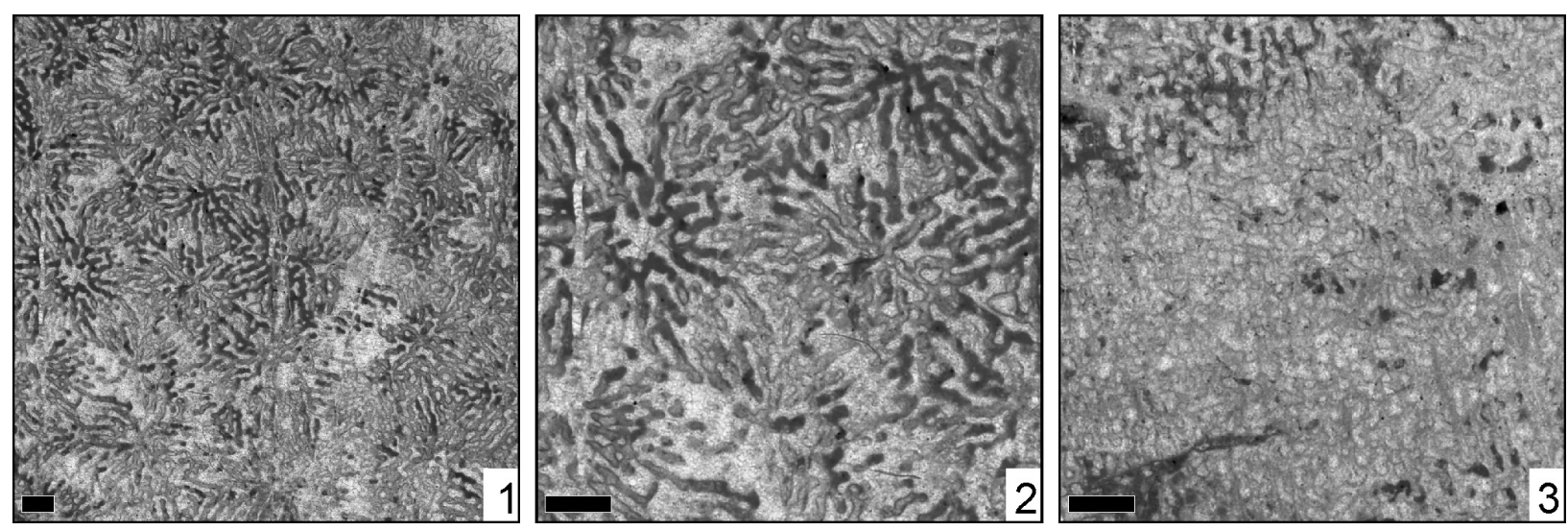

FIGURE 24. Periseris frondescens Orbigny, 1850, MGB 83323. 10: transversal thin section. 11: transversal thin section, detail. 12: longitudinal thin section. Scale $1 \mathrm{~mm}$. 

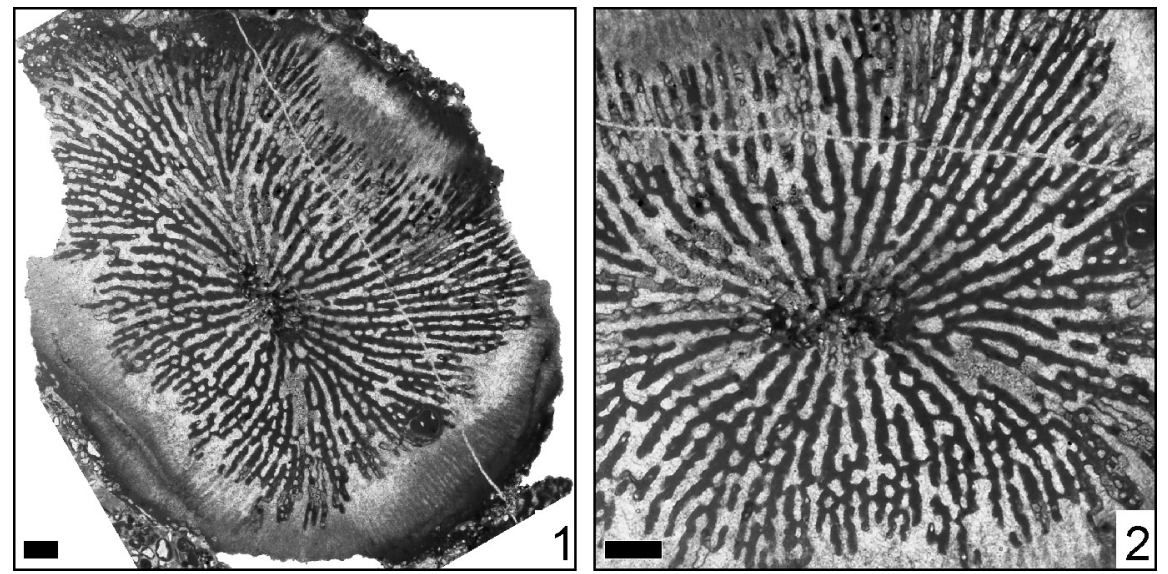

FIGURE 25. Placoseris poculum (Fromentel, 1857), MGB 83260. 1: transversal thin section. 2: transversal thin section, detail. Scale $1 \mathrm{~mm}$.

milia Orbigny, 1849. Acrosmilia is a conceptual genus; the type specimen of the type species is available but so poorly preserved that important diagnostic features, such as the presence or absence of pennulae, cannot be observed. It is therefore recommended to re-establish the use of the genus Leptophyllia that is currently considered a junior synonym of Acrosmilia. The type material of the type species of Leptophyllia is better preserved and moreover, it is easier to obtain topotypical material. The study of type specimens and topotypical material has shown that Leptophyllia is more related to the mainly Late Cretaceous family Synastraeidae and restricted to the Late Cretaceous, whereas Placoseris belongs to the Jurassic to mainly Lower Cretaceous family Latomeandridae. Leptophyllia has thicker and less perforate septa whereas in Placoseris the septa are thinner and more perforate at the inner margin. Moreover, the septa are often connected to each other in the latter, a characteristic that is less common in Leptophyllia (see Löser et al., 2019 for details).

Occurrence. Hauterivian of France (Haute-Marne) Saint Dizier (MNHN nn). Lower Hauterivian (Radiatus Zone) of France (Haute-Marne) Saint Dizier. Lower Cenomanian (Mantelli Zone) of Germany (Nordrhein/Westfalen) Mülheim/Ruhr, Kassenberg (BSPG 2003 XX 1079). Lower Cenomanian (Dixoni Zone) of Spain (Cantabria, Santander) Cobreces, Luaña playa (BSPG 2007 V 372). Middle Cenomanian of Germany (Bayern) Roßstein-Almen (LFU 8336SG015155\#1). Upper Cenomanian (Plenus Zone) of Germany (Sachsen) Dresden-Plauen, Ratssteinbruch, southern quarry. Latest Cenomanian (Juddi Zone) of France (Aude) Les Corbières, Sougraigne, Prat-Périé (BSPG 2011 XXVI 17).
Genus THALAMOCAENIOPSIS Alloiteau, 1954

Type species. Thalamocaeniopsis ouenzensis Alloiteau, 1954, by original designation.

Thalamocaeniopsis explanata (Reig Oriol, 1994) Figure 26

v $1880 \quad$ Isastraea serialis ME and $\mathrm{H}$; Achiardi, $\mathrm{p}$. 247

v $1994 \quad$ Microsolena explanata n. sp.; Reig Oriol, p. 33, pl. 4, fig. 8 , pl. 5, fig. 1

v 1996 Latiastrea cf. kaufmanni (Koby, 1897); Baron-Szabo and Steuber, p. 25, pl. 15, figs. 1,2

v 2018 Thalamocaeniopsis explanata (Reig Oriol, 1994); Löser, Steuber, and Löser, p. 44, pl. 4, figs. 7-9

Material. MGB 83275; 1 thin section.

Dimensions. See Table 21.

Remarks. The genus Thalamocaeniopsis was described and the type material was depicted in Löser (2016). The present material differs slightly from the type material of $T h$. explanata by smaller dimensions. Because of its enlarged corallites the specimen shows a certain affinity to Latiastrea material from the same area, but corallite rows could not be found.

Occurrence. Bathonian of Italy (Veneto, Verona) Monte Pastello. Kimmeridgian of France (Jura) Valfin (MNHN BeauG222). Upper Barremian to Lower Aptian of Poland, Malopolskie (UJ nn). Lower Aptian of Greece (Viotía) Levadia, Perachorion (BSPG 2003 XX 5723); Arachova. Aptian to Albian of Greece (Fokída) Mariolada, S spring Kria Vrissi, trail section (BSPG 2009 XV 21). Upper Aptian of Spain (Valencia, Castellón) Benicasin, La Venta (FGUB LV-31). Upper Aptian (Nolani Zone) of Spain (Cataluña, Barcelona) Garraf, Las Mesquites. Lower Albian of Mexico (Sonora) Tuape, 

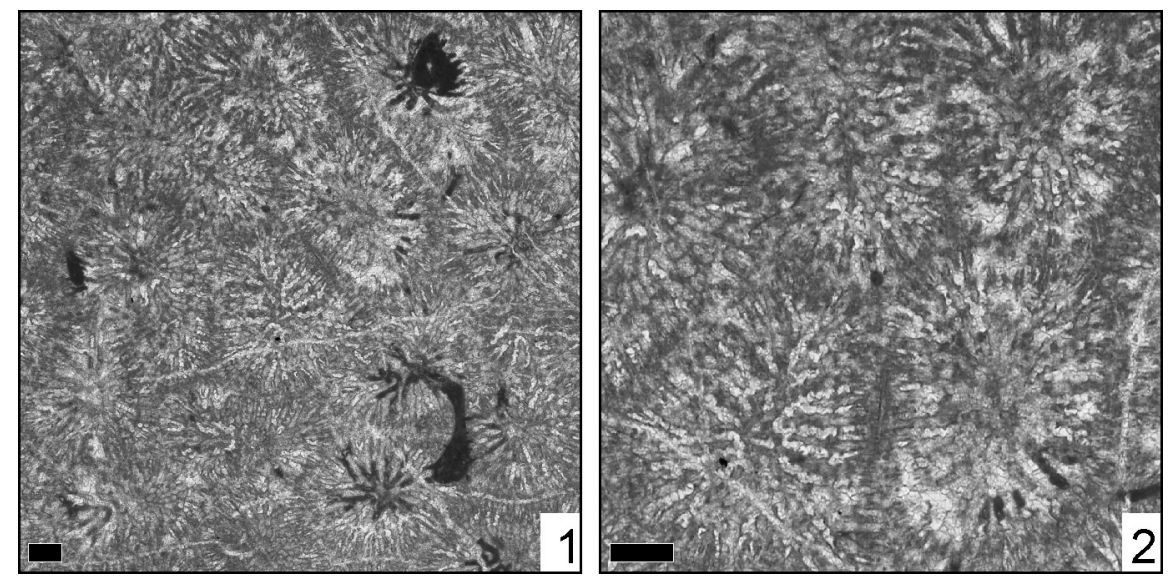

FIGURE 26. Thalamocaeniopsis explanata Reig Oriol, 1994, MGB 83275. 1: transversal thin section. 2: transversal thin section, detail. Scale $1 \mathrm{~mm}$.

TABLE 21. Measurements of MGB 83275.

\begin{tabular}{ccccccc}
\hline & $\mathbf{n}$ & $\mathbf{m i n}-\mathbf{m a x}$ & $\boldsymbol{\mu}$ & $\mathbf{s}$ & $\mathbf{c v}$ & $\boldsymbol{\mu \pm \mathbf { s }}$ \\
\hline clmin & 20 & $2.79-3.88$ & 3.33 & 0.34 & 10.4 & $2.98-3.68$ \\
clmax & 20 & $3.56-5.22$ & 4.48 & 0.52 & 11.5 & $3.96-5.00$ \\
ccd & 20 & $3.15-4.46$ & 3.74 & 0.35 & 9.4 & $3.38-4.09$ \\
$\mathrm{~s}$ & 8 & $51-72$ & 61.6 & 6.67 & 10.8 & $55-68$ \\
\hline
\end{tabular}

Cerro de la Espina (ERNO L-4297); Santa Ana (ERNO L-4407). Lower Cenomanian of Greece (Kozani) Kozani, Nea Nikopoli. Lower Cenomanian (Dixoni Zone) of Spain (Cantabria, Santander) Cobreces, Luaña playa (BSPG 2007 V 328).

Family MICROSOLENIDAE Koby, 1889 Genus EOCOMOSERIS Melnikova, Roniewicz and Loeser, 1993

Type species. Eocomoseris gurumdyensis Roniewicz, 2011 nom. nov. pro Eocomoseris ramosa Melnikova, Roniewicz and Loeser, 1993, by original designation.

Remarks. The genus Eocomoseris was originally only described with one Cretaceous species. Later revisions of type material revealed that there exists another two formally described species. The distinction of species is difficult because the principal characteristic - the distance of the corallite centres - varies within one colony. The material presented here is not well enough preserved to establish new taxa. For the distinction of the four species in the study area see Table 22.

\section{Eocomoseris sp. 1}

Figure 27

v 2012 Eocomoseris raueni Melnikowa et al., 1993; Bover Arnal, Löser and Moreno Bedmar, p. 58 , figs. $11 \mathrm{I}-\mathrm{K}$
TABLE 22. Distinction of the Eocomoseris species within the study area.

\begin{tabular}{cccc}
\hline $\begin{array}{c}\text { Small lumen } \\
(\mathbf{m m})\end{array}$ & $\begin{array}{c}\text { Corallite } \\
\text { distance } \\
(\mathbf{m m})\end{array}$ & $\begin{array}{c}\text { Number of } \\
\text { septa }\end{array}$ & Species \\
\hline $1.2-1.5$ & $1.6-2.1$ & $21-25$ & sp. 1 \\
$1.7-2.2$ & $1.8-2.5$ & $14-18$ & sp. 2 \\
$2.6-3.0$ & $2.4-3.7$ & $21-23$ & sp. 3 \\
$2.7-3.3$ & $2.9-3.9$ & $23-27$ & sp. 4 \\
\hline
\end{tabular}

v 2013 Eocomoseris raueni Melnikova et al. 1993; Löser, Castro and Nieto, p. 25, pl. 8, figs. $11-12$

Material. MGB 83343, 83347; 4 thin sections.

Dimensions. See Table 23.

Occurrence. Valanginian to Aptian of Mexico (Puebla) San Juan Raya (IGM 9196). Lower Aptian (Furcata Zone) of Spain (Aragón, Teruel) Teruel, La Serna. Albian to Lower Cenomanian of UK (Devonshire) Branscombe, Culverhole (NHM 49). Lower Upper Albian (Inflatum Zone) of Spain (Valencia, Alicante) Sierra de Llorençá. Lower Cenomanian (Dixoni Zone) of Spain (Cantabria, Santander) Cobreces, Luaña playa (BSPG 2007 V 412). Upper Cenomanian (Geslinianum Zone) of Portugal (Coimbra) Carrasqueira (ERNO L-132314).

\section{Eocomoseris sp. 2}

\section{Figure 28}

v 2009 Eocomoseris raueni Löser, 1993; Morycowa and Masse, p. 112, fig. 9

v 2017 Eocomoseris sp.; Löser and Bilotte, p. 13, figs. $9 \mathrm{~g}$-i

Material. MGB 83247; 2 thin sections.

Dimensions. See Table 24.

Occurrence. Valanginian of Switzerland (Vaud) Arzier (MGL nn). Valanginian to Aptian of Mexico 

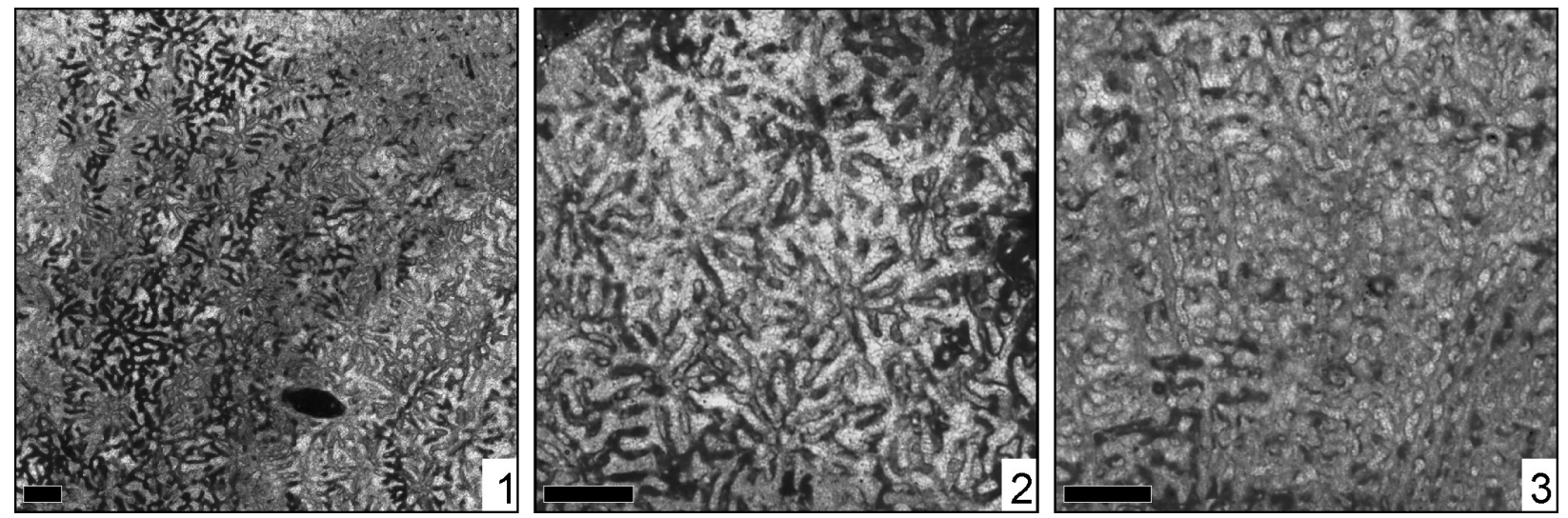

FIGURE 27. Eocomoseris sp. 1, MGB 83343. 1: transversal thin section. 2: transversal thin section, detail. 3: longitudinal thin section. Scale $1 \mathrm{~mm}$.

TABLE 23. Measurements of MGB 83343.

\begin{tabular}{ccccccc}
\hline & $\mathbf{n}$ & $\mathbf{m i n}-\mathbf{m a x}$ & $\boldsymbol{\mu}$ & $\mathbf{s}$ & $\mathbf{c v}$ & $\boldsymbol{\mu \pm \mathbf { s }}$ \\
\hline $\operatorname{clmin}$ & 20 & $0.98-1.54$ & 1.32 & 0.16 & 12.3 & $1.16-1.48$ \\
$\operatorname{clmax}$ & 20 & $1.39-2.17$ & 1.75 & 0.21 & 12.2 & $1.54-1.97$ \\
$\operatorname{ccd}$ & 20 & $1.33-2.41$ & 1.85 & 0.27 & 14.6 & $1.58-2.12$ \\
$\mathrm{~s}$ & 6 & $19-26$ & 23.0 & 2.44 & 10.6 & $21-25$ \\
\hline
\end{tabular}

(Puebla) San Juan Raya (IGM 9208). Lower Upper Barremian of France (Bouches-du-Rhône) Chainon la Fare, Saint Chamas, canal EDF. Cenomanian of Greece (Fokída) Kiona massif, Panourgias [= Dremisa] (BSPG 2003 XX 5902). Lower Cenomanian (Dixoni Zone) of Spain (Cantabria, Santander) Cobreces, Luaña playa (BSPG 2007 V 028). Latest Cenomanian (Juddi Zone) of France (Aude) Les Corbières, Col de Escudiés (UPS HL 008).

\section{Eocomoseris sp. 3}

Figure 29

Material. MGB 83234; 1 thin section.
TABLE 24. Measurements of MGB 83247

\begin{tabular}{ccccccc}
\hline & $\mathbf{n}$ & $\mathbf{m i n}-\mathbf{m a x}$ & $\boldsymbol{\mu}$ & $\mathbf{s}$ & $\mathbf{c v}$ & $\boldsymbol{\mu \pm \mathbf { s }}$ \\
\hline clmin & 6 & $1.85-2.33$ & 2.04 & 0.17 & 8.4 & $1.86-2.21$ \\
$\operatorname{clmax}$ & 6 & $1.74-2.36$ & 2.08 & 0.21 & 10.1 & $1.86-2.29$ \\
$\operatorname{ccd}$ & 10 & $1.83-2.73$ & 2.14 & 0.33 & 15.8 & $1.80-2.48$ \\
$\mathrm{~s}$ & 5 & $14-18$ & 16.0 & 1.58 & 9.8 & $14-18$ \\
\hline
\end{tabular}

v 1880 Microsolena (?) spec.; Toula, p. 254, pl. 6, fig. 12

v 2009 Eocomoseris sp.; Löser, Stemann, and Mitchell, p. 343, figs. 6.11-6.12

v 2018 Eocomoseris sp.; Löser, Steuber, and Löser, p. 45, pl. 5, figs. 1-3

Dimensions. See Table 25.

Occurrence. Hauterivian of Jamaica (Saint Catharine) Benbow Inlier, Copper. Lower Cenomanian of Greece (Kozani) Kozani, Nea Nikopoli; Serbia (East Serbia) Pirot, Modrestena.

\section{Eocomoseris sp. 4}

Figure 30
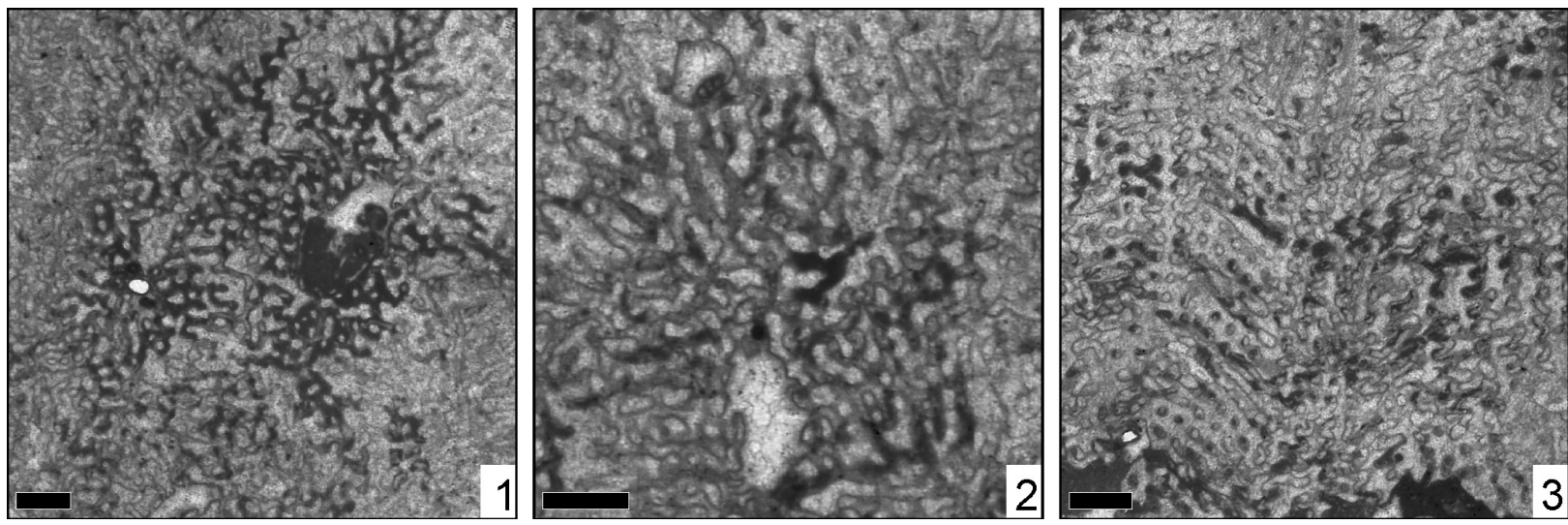

FIGURE 28. Eocomoseris sp. 2, MGB 83247. 1: transversal thin section. 2: transversal thin section, detail. 3: longitudinal thin section. Scale $1 \mathrm{~mm}$. 

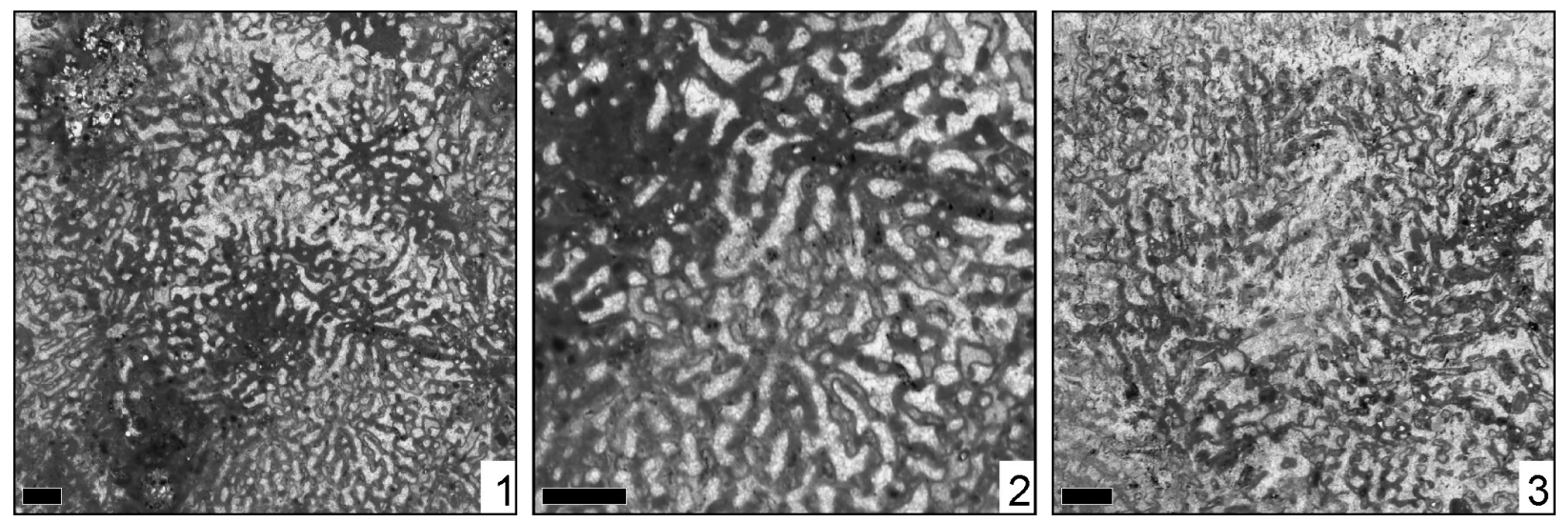

FIGURE 29. Eocomoseris sp. 3, MGB 83234. 1: transversal thin section. 2: transversal thin section, detail. 3: longitudinal thin section. Scale $1 \mathrm{~mm}$.

TABLE 25. Measurements of MGB 83234.

\begin{tabular}{ccccccc}
\hline & $\mathbf{n}$ & $\min -\max$ & $\boldsymbol{\mu}$ & $\mathbf{s}$ & $\mathbf{c v}$ & $\boldsymbol{\mu} \pm \mathbf{s}$ \\
\hline clmin & 5 & $2.58-3.03$ & 2.82 & 0.18 & 6.6 & $2.64-3.01$ \\
$\operatorname{clmax}$ & 5 & $2.68-4.22$ & 3.34 & 0.62 & 18.6 & $2.72-3.97$ \\
$\operatorname{ccd}$ & 10 & $2.37-3.99$ & 3.05 & 0.61 & 19.9 & $2.44-3.66$ \\
$\mathrm{~s}$ & 10 & $20-24$ & 22.1 & 1.28 & 5.8 & $21-23$ \\
\hline
\end{tabular}

Material. MGB 83231; 2 thin sections.

Dimensions. See Table 26.

Remarks. This specimen compares well to Eocomoseris sp. 3 in its corallite dimensions but clearly has a higher number of septa.

Genus MEANDRARAEA Etallon, 1859

Type species. Meandraraea marcouana Etallon, 1859 , by subsequente definition.

Meandraraea miyakoensis Eguchi, 1951

Figure 31

Material. MGB 83226; 1 thin section.
TABLE 26. Measurements of MGB 83231.

\begin{tabular}{ccccccc}
\hline & $\mathbf{n}$ & $\min -\mathbf{m a x}$ & $\boldsymbol{\mu}$ & $\mathbf{s}$ & $\mathbf{c v}$ & $\boldsymbol{\mu \pm \mathbf { s }}$ \\
\hline clmin & 20 & $2.48-3.51$ & 2.97 & 0.32 & 10.9 & $2.65-3.30$ \\
clmax & 20 & $3.03-3.61$ & 3.33 & 0.16 & 4.9 & $3.17-3.49$ \\
ccd & 20 & $2.73-4.35$ & 3.40 & 0.49 & 14.6 & $2.90-3.90$ \\
$\mathrm{~s}$ & 5 & $23-28$ & 25.0 & 2.34 & 9.3 & $23-27$ \\
\hline
\end{tabular}

vp 1898 Maeandraraea maeandroides; Koby, p. 85, pl. 20, figs. 3-6

v*1951 Meandrarea miyakoensis Eguchi, p. 38, pl. 9 , fig. 8

Dimensions. See Table 27.

Remarks. The genus was recently described in larger detail (Löser, 2016). The corallite series are short, as in the type species of the genus. The name Maeandraraea maeandroides cannot be applied to our material because the type series of this species consists of five syntypes that represent different species. A lectotype has not been designated.
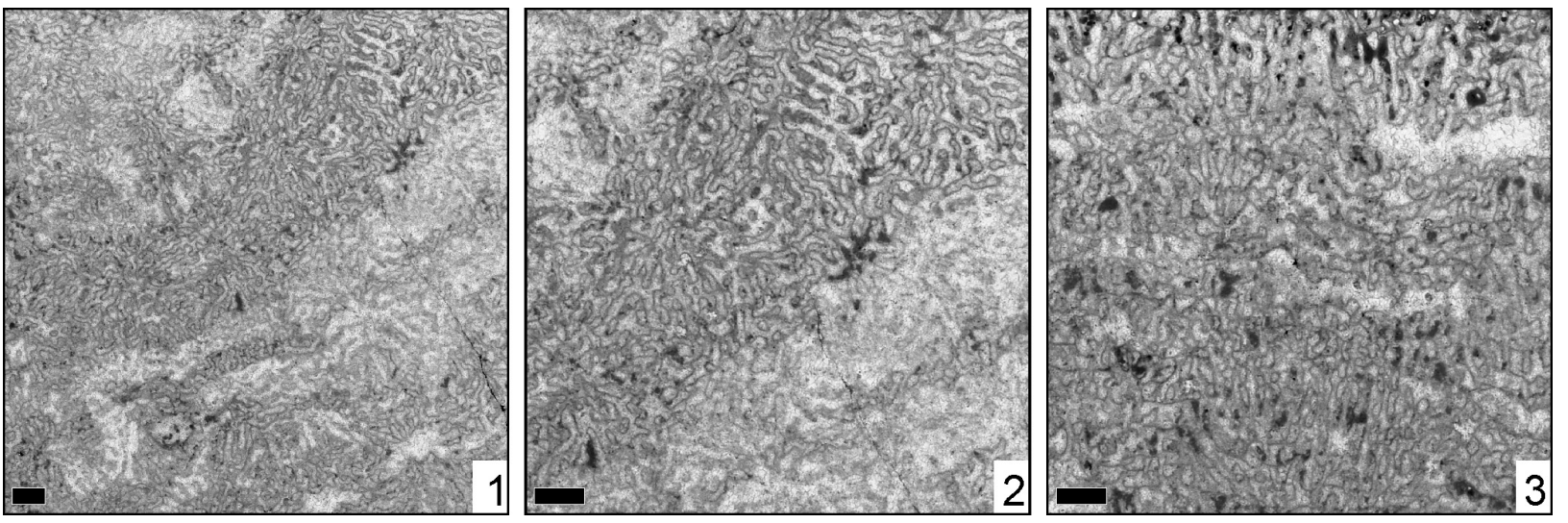

FIGURE 30. Eocomoseris sp. 4, MGB 83231. 1: transversal thin section. 2: transversal thin section, detail. 3: longitudinal thin section. Scale $1 \mathrm{~mm}$. 

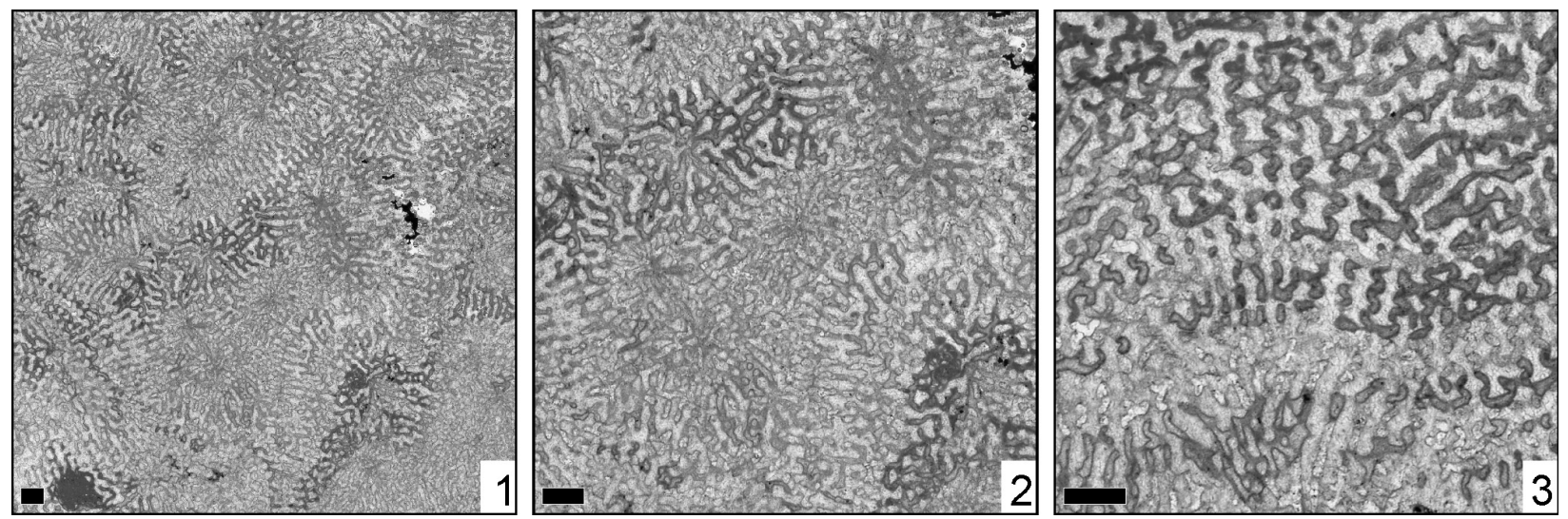

FIGURE 31. Meandraraea miyakoensis Eguchi, 1951, MGB 83226. 1: transversal thin section. 2: transversal thin section, detail. 3: longitudinal thin section. Scale $1 \mathrm{~mm}$.

TABLE 27. Measurements of MGB 83226.

\begin{tabular}{ccccccc}
\hline & $\mathbf{n}$ & $\min -\mathbf{m a x}$ & $\boldsymbol{\mu}$ & $\mathbf{s}$ & $\mathbf{c v}$ & $\boldsymbol{\mu \pm \mathbf { s }}$ \\
\hline $\mathrm{crd}$ & 10 & $2.44-4.06$ & 3.37 & 0.50 & 14.8 & $2.87-3.87$ \\
$\mathrm{cdw}$ & 12 & $2.13-3.12$ & 2.63 & 0.29 & 11.0 & $2.34-2.92$ \\
$\mathrm{~s}$ & 10 & $17-23$ & 19.7 & 1.70 & 8.6 & $18-21$ \\
\hline
\end{tabular}

Occurrence. Callovian to Kimmeridgian of Japan (Kochi-ken) Takaoka-gun, Sakawa-cho, Kamo, Mitoda (TUM 38443). Kimmeridgian of Spain (Iberian Chaines) 70 Moscardón (RUB MK25). Barremian of France (Doubs) Morteau. Latest Aptian of Japan (Iwate-ken) Shimohei-gun, Tanohata-mura, Koikorobe.

Genus MICROSOLENA Lamouroux, 1821

Type Species. Microsolena porosa Lamouroux, 1821 , by monotypy.

Microsolena interjecta Alloiteau, 1958

Figure 32
TABLE 28. Measurements of MGB 83338.

\begin{tabular}{ccccccc}
\hline & $\mathbf{n}$ & $\mathbf{m i n}-\mathbf{m a x}$ & $\boldsymbol{\mu}$ & $\mathbf{s}$ & $\mathbf{c v}$ & $\boldsymbol{\mu \pm \mathbf { s }}$ \\
\hline ccd & 20 & $1.87-2.92$ & 2.30 & 0.29 & 12.7 & $2.00-2.59$ \\
$\mathrm{~s}$ & 5 & $31-38$ & 34.8 & 2.58 & 7.4 & $32-37$ \\
\hline
\end{tabular}

v $1958 \quad$ Microsolena interjecta Alloiteau, p. 90, pl. 1: 1, pl. 15: 6

v 2018 Microsolena ? interjecta Alloiteau, 1958; Löser, Steuber, and Löser, p. 45, pl. 5, figs. 4-6

Material. MGB 83338; 2 thin sections.

Dimensions. See Table 28.

Remarks. The genus was recently described in larger detail (Löser, 2016) and the species in Löser et al. (2018).

Occurrence. Bathonian of Madagascar, W Ampakabo (MNHN M05096). Lower Cenomanian of France (Charente-Maritime) Fouras (BSPG 2003 XX 5599); Greece (Kozani) Kozani, Nea Nikopoli. Upper Cenomanian of France (Bouches-du-
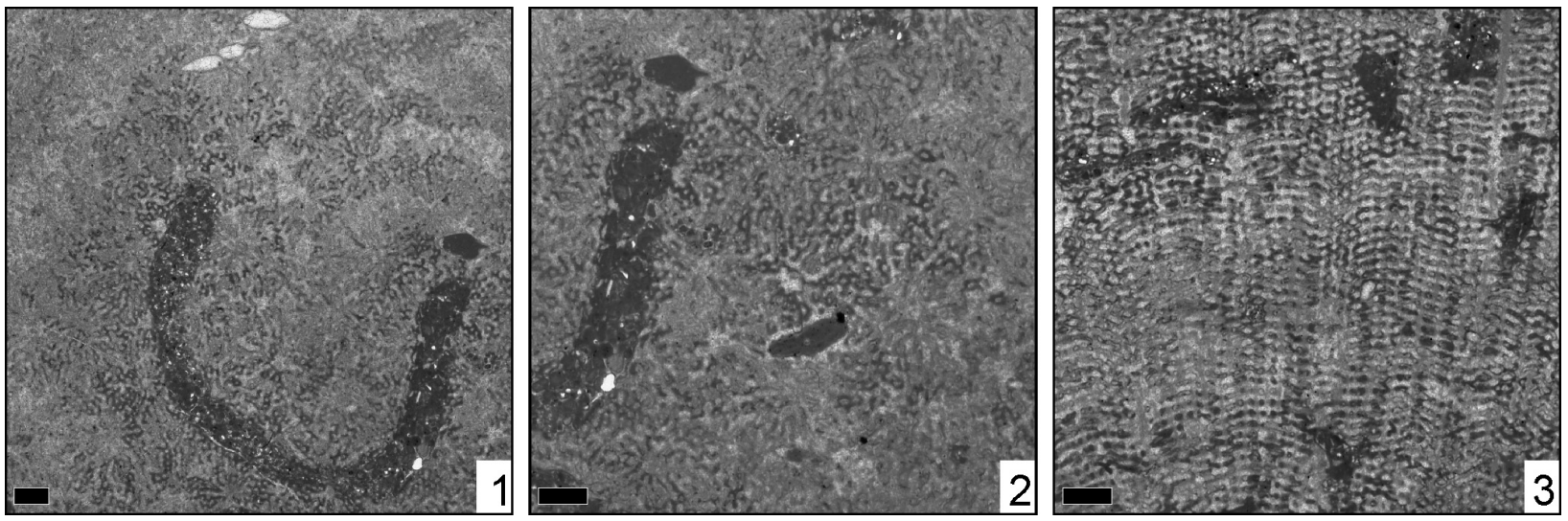

FIGURE 32. Microsolena interjecta Alloiteau, 1958, MGB 83338. 10: transversal thin section. 11: transversal thin section, detail. 12: longitudinal thin section. Scale $1 \mathrm{~mm}$. 

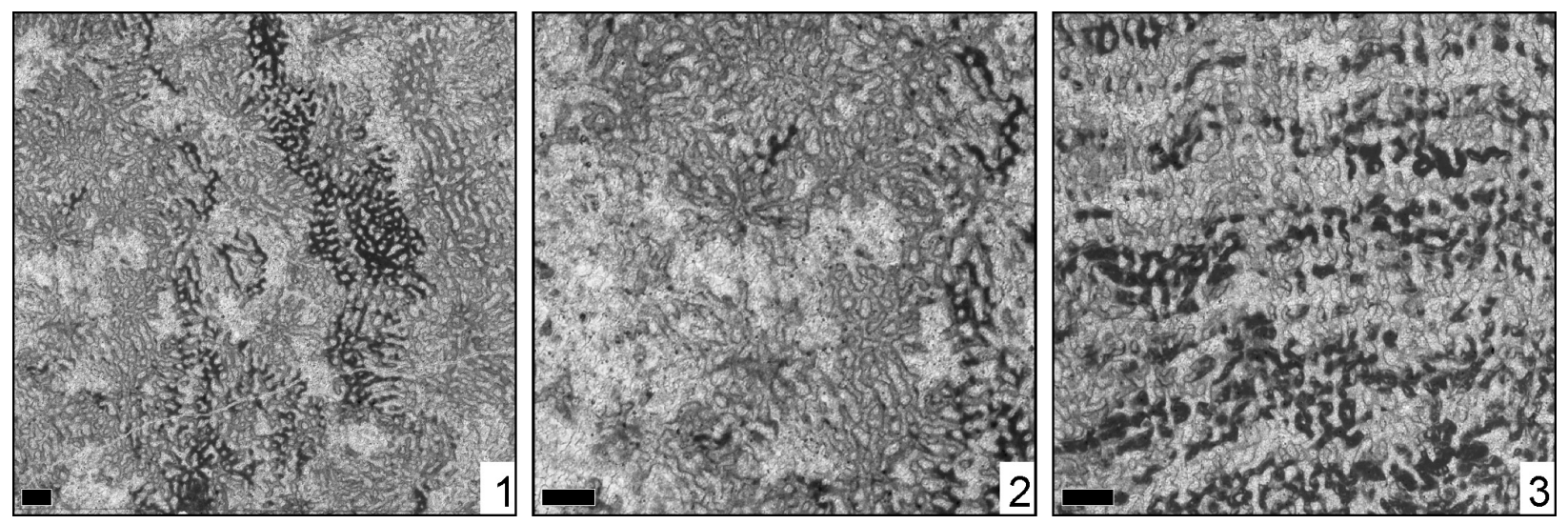

FIGURE 33. Microsolena sp. 1, MGB 83335. 1: transversal thin section. 2: transversal thin section, detail. 3: longitudinal thin section. Scale $1 \mathrm{~mm}$.

Rhône) Martigues, trench along road between Martigues and La Couronne (BSPG 2003 XX 5399).

\section{Microsolena sp. 1}

Figure 33

? 1985 Microsolena foliosa Roniewicz; Geyer and Rosendahl, p. 167, pl. 1, fig. 5

v $1994 \quad$ Microsolena kugleri Wells 1948; Löser, p. 56 , text-figs. 46,47 , pl. 1 , fig. 1 , pl. 8 , fig. 2 , pl. 11 , fig. 4

v 1997 Microsolena kobyi Prever, 1909; BaronSzabo, p. 83, pl. 13, fig. 4

v 1998 Microsolena insignis (Duncan 1879); Schöllhorn, p. 101, pl. 25, fig. 2

v 2003 Meandrophyllia lotharinga (Michelin, 1843); Baron-Szabo and González León, p. 212, fig. 8A

v 2009 Microsolena aff. crassisepta Sikharulidze, 1985; Löser, Stemann and Mitchell, p. 343, figs. 7.6, 7.9

Material. MGB 83335; 2 thin sections.

Dimensions. See Table 29.

Occurrence. Upper Oxfordian of Poland, Staniewice (ZPAL Hiii1211). Hauterivian of Jamaica (Saint Catharine) Benbow Inlier, Copper. Upper Barremian of Poland (Malopolskie, Tarnów) Tarnów, Trzemesna (UJ 4P nn). Upper Barremian to Lower Aptian (Sartousi - Weissi Zone) of Germany (Bayern) Allgäuer Helvetikum, Tiefenbach, Kiesgrube Schwarzenberg (BSPG 1994 XI 186). Upper Barremian to Lower Aptian (Lenticularis Zone) of Mexico (Sonora) Ures, Cerro de Oro. Lower Upper Aptian of Spain (Cataluña, Lérida) Alt

TABLE 29. Measurements of MGB 83335.

\begin{tabular}{ccccccc}
\hline & $\mathbf{n}$ & $\mathbf{m i n}-\mathbf{m a x}$ & $\boldsymbol{\mu}$ & $\mathbf{s}$ & $\mathbf{c v}$ & $\boldsymbol{\mu \pm \mathbf { s }}$ \\
\hline ccd & 25 & $2.57-4.96$ & 3.61 & 0.68 & 18.9 & $2.92-4.29$ \\
$\mathrm{~s}$ & 10 & $27-38$ & 32.5 & 3.53 & 10.8 & $29-36$ \\
\hline
\end{tabular}

Urgell, Sta. Fé, Font Bordonera. Earlymost Albian (Tardefurcata Zone) of Spain (Cataluña, Tarragona) Baix Penedès, Masarbones, field N (BSPG 2003 $X X$ 6022). Lower Albian of Mexico (Sonora) Agua Prieta, E San Bernardino Valley, Cordon Caloso (ERNO L-4445). Lower Cenomanian (Mantelli Zone) of Germany (Nordrhein/Westfalen) Mülheim/ Ruhr, Kassenberg (BSPG 2003 XX 1005). Lower Cenomanian of France (Charente-Maritime) Fouras (ERNO L-5596). Lower Cenomanian (Dixoni Zone) of Germany (Sachsen) Meißen-Zscheila, Trinitatis church (ERNO L-6152). Upper Turonian to Lower Coniacian of Austria (Tirol) Brandenberg, Haidach.

\section{Microsolena sp. 2}

Figure 34

vp 1879 Thamnastraea Ramsayi; Duncan, p. 92, pl. 8, fig. 6

v 1974 Microsolena distefanoi (Prever); Turnšek and Buser, p. 21, 37, pl. 11, fig. 2

v1997 Thamnoseris arborescens Felix, 1891; Baron-Szabo, p. 88, pl. 16, figs. 2, 4

v 2003 Microsolena kobyi Prever, 1909; BaronSzabo and González León, p. 215, fig. 7A

v 2006 Microsolena sp.; Löser, p. 44, fig. 4G

v 2014 Actinaraea tenuis Morycowa, 1971; BaronSzabo, pl. 58, fig. 3, 59, figs. 1, 2

Material. MGB 83342; 2 thin sections.

Dimensions. See Table 30.

Remarks. The present material compares in its corallite dimensions to Microsolena haldonensis (Duncan, 1879), but has fewer septa.

Occurrence. Valanginian to Aptian of Mexico (Puebla) San Juan Raya (IGM). Barremian of France (Doubs) Morteau (MHNN 26777); Mexico (Puebla) Tehuacán, San Antonio Texcala. Upper Barremian to Lower Aptian (Sartousi - Weissi Zone) of Germany (Bayern) Allgäuer Helvetikum, 

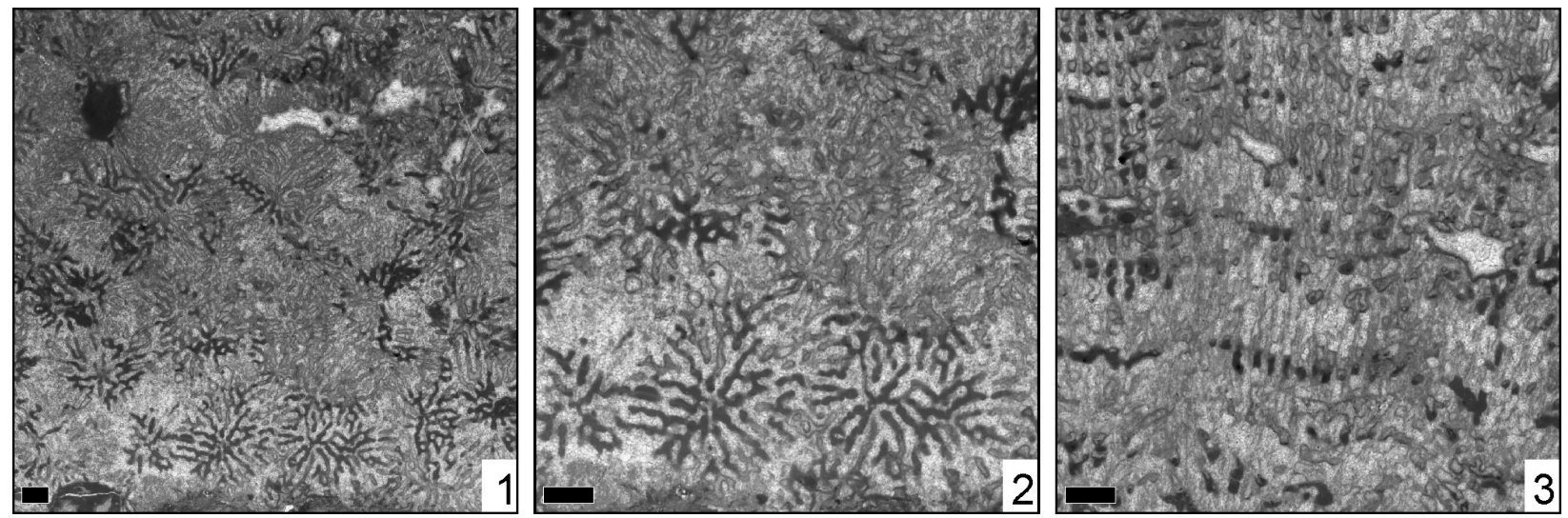

FIGURE 34. Microsolena sp. 2, MGB 83342. 1: transversal thin section. 2: transversal thin section, detail. 3: longitudinal thin section. Scale $1 \mathrm{~mm}$.

TABLE 30. Measurements of MGB 83342

\begin{tabular}{ccccccc}
\hline & $\mathbf{n}$ & $\mathbf{m i n}-\mathbf{m a x}$ & $\boldsymbol{\mu}$ & $\mathbf{s}$ & $\mathbf{c v}$ & $\boldsymbol{\mu \pm \mathbf { s }}$ \\
\hline ccd & 20 & $2.42-3.56$ & 2.97 & 0.33 & 11.3 & $2.63-3.31$ \\
$\mathrm{~s}$ & 15 & $23-34$ & 28.8 & 3.48 & 12.1 & $25-32$ \\
\hline
\end{tabular}

Brandalpe (BSPG $1997 \vee$ 1). Lower Aptian of Slovenia (West Slovenia) Banskja Planota, Osojnica. Earlymost Albian (Tardefurcata Zone) of Spain (Cataluña, Barcelona) Alt Penedès, Castellvi de la Marca, Can Pascual (BSPG 2003 XX 6275). Lower Albian of Mexico (Sonora) Agua Prieta, E San Bernardino Valley, Cordon Caloso (ERNO L-4203); Ures, Cerro de Oro (ERNO 3092). Upper Albian of UK (Devonshire) Exeter, Haldon Hill. Lower Cenomanian (Dixoni Zone) of Spain (Cantabria, Santander) Cobreces, Luaña playa (BSPG 2007 V 326). Upper Turonian to Lower Coniacian of Austria (Tirol) Brandenberg, Haidach.
Superfamily EUGYROIDEA Achiardi, 1875 Family SOLENOCOENIIDAE Roniewicz, 2008 Genus BILATEROCOENIA Morycowa, 1974

Type species. Bilaterocoenia hexaseptata Morycowa, 1974, by original designation.

\section{Bilaterocoenia sp.}

Figure 35

Material. MGB 83281; 1 thin section.

Dimensions. See Table 31.

Remarks. The genus Bilaterocoenia is well known but rare. Three species are formally described; the present specimen differs from all known species by the very small corallite dimensions and could represent a new species.

\section{Genus CONFUSAFORMA Löser, 1987}

Type species. Confusaforma weyeri Löser, 1987, by original designation.

\section{Confusaforma prima sp. nov. Löser} Figure 36

\section{zoobank.org/305E2A06-753B-499D-981B-6B12BB3D01B9}
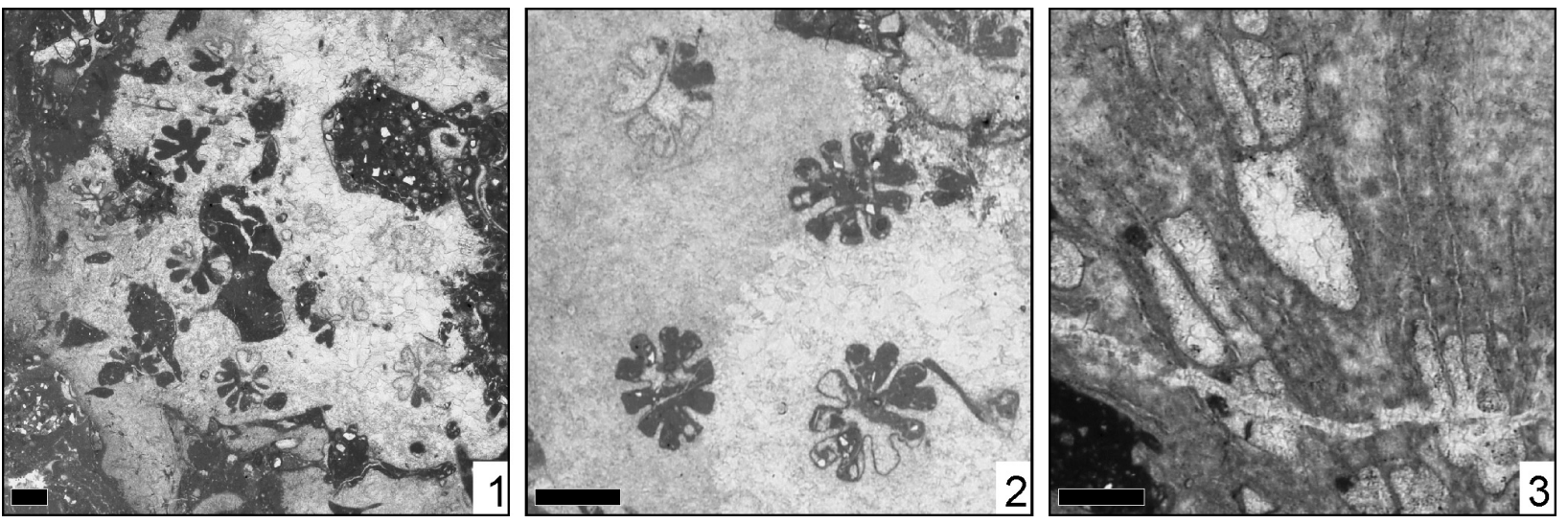

FIGURE 35. Bilaterocoenia sp., MGB 83281. 1: transversal thin section. 2: transversal thin section, detail. 3: longitudinal thin section. Scale $1 \mathrm{~mm}$. 
TABLE 31. Measurements of MGB 83281.

\begin{tabular}{ccccccc}
\hline & $\mathbf{n}$ & $\min -\mathbf{m a x}$ & $\boldsymbol{\mu}$ & $\mathbf{s}$ & $\mathbf{c v}$ & $\boldsymbol{\mu \pm \mathbf { s }}$ \\
\hline clmin & 18 & $1.09-1.61$ & 1.36 & 0.13 & 9.5 & $1.23-1.49$ \\
clmax & 18 & $1.28-2.09$ & 1.66 & 0.25 & 15.4 & $1.41-1.92$ \\
ccd & 15 & $1.74-2.91$ & 2.42 & 0.34 & 14.1 & $2.08-2.77$ \\
$\mathrm{~s}$ & 15 & $8-12$ & 10.06 & 0.79 & 7.9 & $9-11$ \\
\hline
\end{tabular}

v 2003 Confusaforma weyeri Löser, 1987; BaronSzabo and González León, p. 207, fig. 7B

v 2013 Confusaforma sp.; Löser, Castro and Nieto, p. 29 , pl. 9 , figs. $10-12$

v 2015 Confusaforma aff. weyeri Löser, 1987; Löser, p. 17, figs. 1D-F

Etymology. Prima, the first. The species is the earliest record of the genus.

Holotype. MGB 83346 with two thin sections.

Type locality. Puerto Llano section, Cabañas, Sierra de Cazorla, Jaén, Andalucía, Spain.

Type level. Sierra del Pozo Fm, Lower Valanginian.

Depository. Museo de Geología de Barcelona, Spain.

Diagnosis. Confusaforma with an average small calicular diameter of $1.09 \mathrm{~mm}$ and an average large calicular diameter of $1.29 \mathrm{~mm}$. The septal number varies between three and five.

Material. MGB 83346; 2 thin sections.

Dimensions. See Table 32.

Description. Cerioid colony. Corallite outline irregular. Septa compact. Microstructure of septa unknown. Septa in cross section externally thick and of triangular outline. Symmetry of septa irregular. Septa very short, reduced to ridges, not connected to each other. Septal lateral face smooth, inner margin smooth. Pali, costae, synapticulae, and columella absent. Endotheca consists of numerous and regular tabulae. Wall compact, but its structure is unknown. Budding extracalicinal.

Comparison. From Confusaforma weyeri the new species distinguishes by larger calicular dimensions. Even larger dimensions show material

TABLE 32. Measurements of MGB 83346

\begin{tabular}{ccccccc}
\hline & $\mathbf{n}$ & $\mathbf{m i n}-\mathbf{m a x}$ & $\boldsymbol{\mu}$ & $\mathbf{s}$ & $\mathbf{c v}$ & $\boldsymbol{\mu \pm \mathbf { s }}$ \\
\hline clmin & 30 & $0.87-1.30$ & 1.09 & 0.12 & 11.4 & $0.96-1.21$ \\
$\operatorname{clmax}$ & 30 & $1.02-1.50$ & 1.29 & 0.12 & 9.8 & $1.17-1.42$ \\
$\operatorname{ccd}$ & 30 & $1.85-2.48$ & 2.14 & 0.17 & 8.1 & $1.97-2.32$ \\
$\mathrm{~s}$ & 30 & $3-5$ & 4.0 & 0.69 & 17.3 & $3-5$ \\
\hline
\end{tabular}
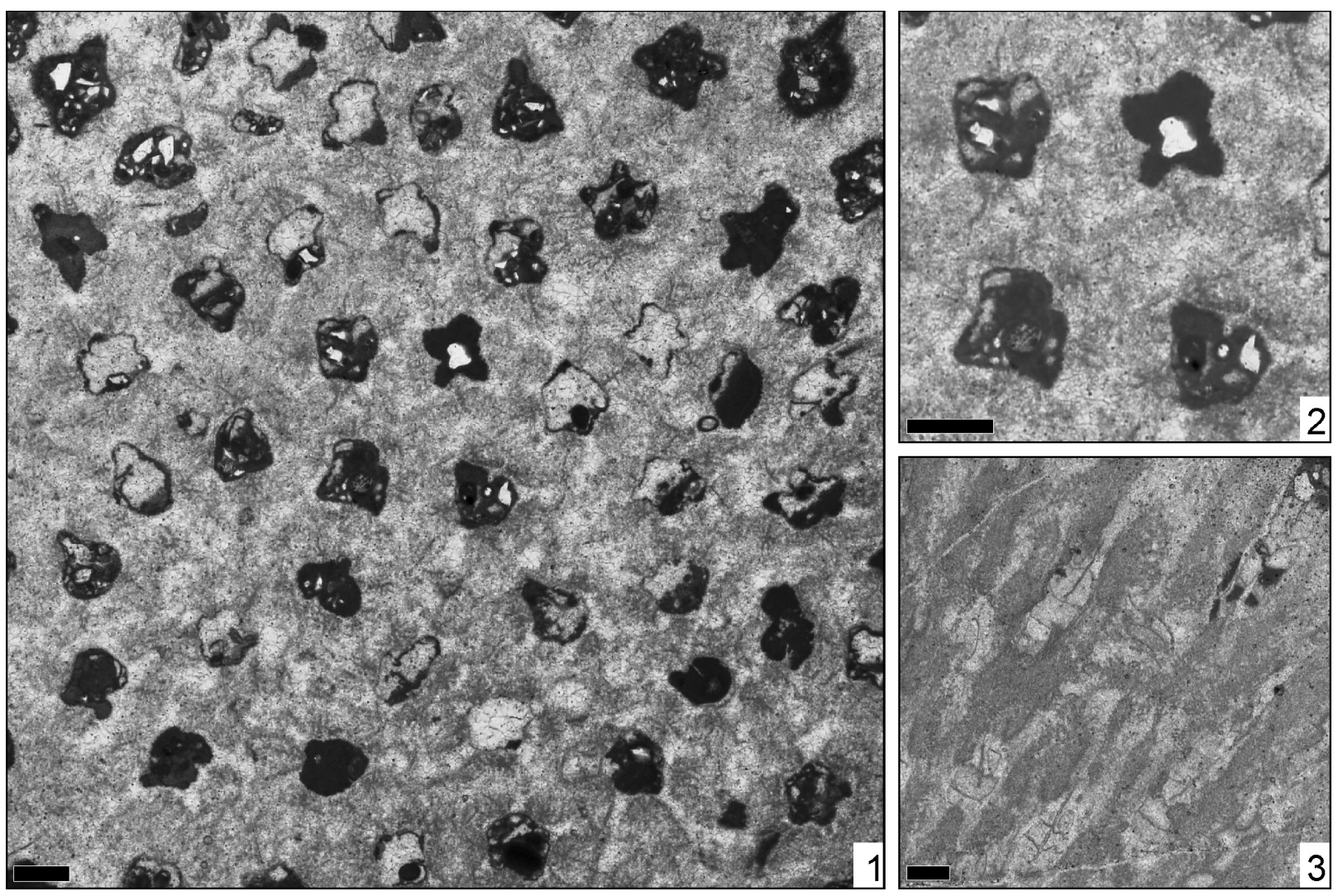

FIGURE 36. Confusaforma prima sp. nov. Löser, MGB 83346. 1: transversal thin section. 2: transversal thin section, detail. 3: longitudinal thin section. Scale $1 \mathrm{~mm}$. 

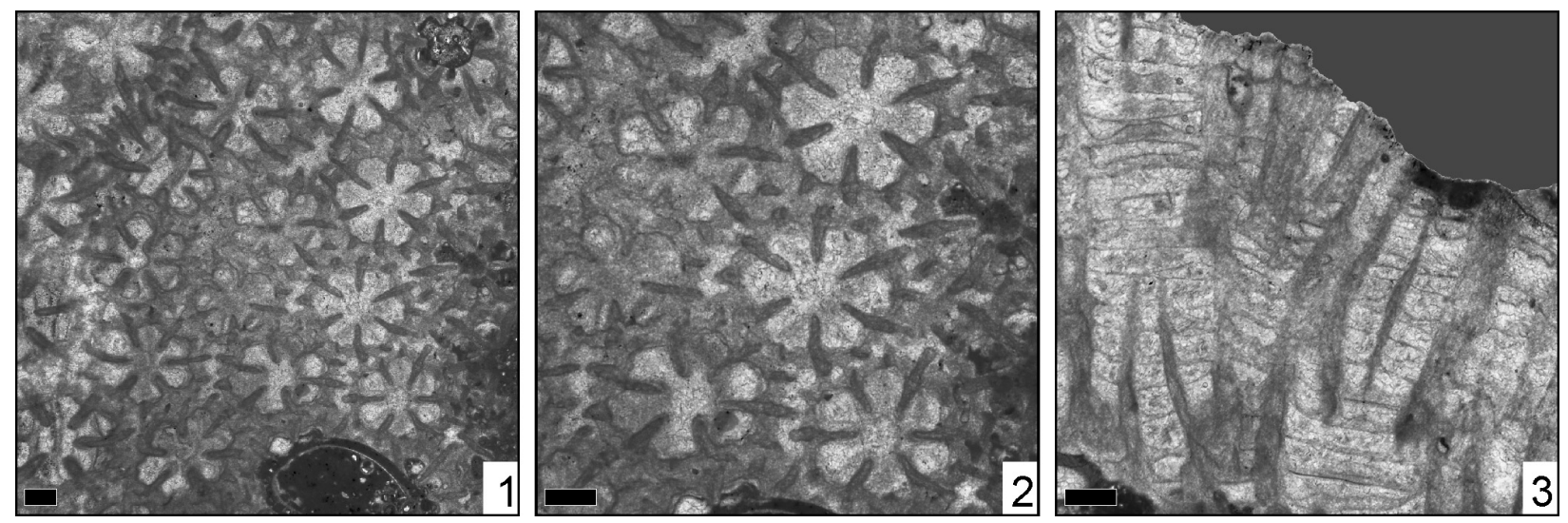

FIGURE 37. Cryptocoenia neocomiensis Orbigny, 1850, MGB 83310. 1: transversal thin section. 2: transversal thin section, detail. 3: Iongitudinal thin section. Scale $1 \mathrm{~mm}$.

described by Löser, Castro and Nieto (2013) from the Upper Albian of southern Spain. C. carpathica Kolodziej, 1995, does not belong to this genus.

Occurrence. Lower Aptian of Italy (Abruzzi, L'Aquila) Monti d'Ocre, Sotto Colle Pagliare (PU 18162). Lower Albian of Mexico (Sonora) Tuape, Cerro de la Espina. Lower Upper Albian (Inflatum Zone) of Spain (Valencia, Alicante) Sierra de Llorençá.

\section{Genus CRYPTOCOENIA Orbigny, 1849}

Type species. Astrea alveolata Goldfuss, 1826, by monotypy.

\section{Cryptocoenia neocomiensis Orbigny, 1850,} Figure 37

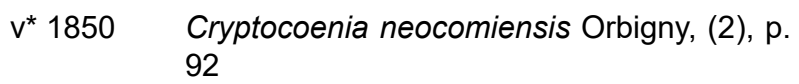

v 1881 Convexastrea bachmanni Koby; Koby, p. 103, pl. 23, fig. 5

v 1881 Convexastrea meriani; Koby, p. 102, pl. 23, figs. 1-4

v 1881 Convexastrea bachmanni Koby; Koby, p. 103, pl. 23, fig. 5

v 1897 Cryptocoenia Picteti; Koby, p. 32, pl. 2, figs. 11, 11a

v 1966 Adelocoenia bachmanni Koby; Beauvais, p. 992, pl. 2, fig. 1

v1971 Pseudocoenia annae (Volz, 1903); Morycowa, p. 42

v 2006 Cryptocoenia corbariensis (Alloiteau, 1948); Löser, p. 15, fig. 2A

v 2016 Cryptocoenia neocomiensis Orbigny, 1850; Löser, fig. $\mathrm{C} 61$

Material. MGB 83310; 2 thin sections.

Dimensions. See Table 33.

Remarks. Cryptocoenia is a common Jurassic and Early Cretaceous coral genus. It was recently described and depicted (Löser, 2016). Cryptocoe- nia neocomiensis is very closely related to Cryptocoenia regularis (Fromentel, 1884), if not even synonymous. The type specimens of both type species derive from the same area (the Hauterivian of the Paris Basin).

Occurrence. Bathonian of Switzerland (Bern) Boltigen. Lower Kimmeridgian of Spain (Iberian Chaines) 08 Torrecilla, old road, northern wing (RUB V12-1). Valanginian to Aptian of Mexico (Puebla) San Juan Raya (IGM 9221). Upper Valanginian to Lower Hauterivian of Mexico (Puebla) San Juan Raya, Arroyo San Francisco (ERNO L-nn). Lower Hauterivian (Radiatus Zone) of France (Aube) Marolles (MHNG 4701); Troyes, Vallières (CF 154); France (Haute-Marne) Saint Dizier; France (Yonne) Fontenoy, field $S$ the junction to Les Merles (BSPG 2003 XX 5037); Leugny, Les Cassines $4 \mathrm{~km}$ E Leugny (BSPG 2003 XX 5098). Barremian of France (Doubs) Morteau; Mexico (Puebla) Tehuacán, San Antonio Texcala (GPSL FLX 2090). Barremian to Lower Aptian of Romania (Suceava) Pojorîta area, CîmpulungMoldovenesc, Rarau Mt. Lower Aptian (Furcata Zone) of Spain (País Vasco, Vizcaya) Bilbao, Peñascal, $1.5 \mathrm{~m}$ below top of the $\mathrm{Fm}$, in the cutting of the quarry (ERNO L-140306). Earlymost Albian (Tardefurcata Zone) of Spain (Cataluña, Tarragona) Baix Penedés, Marmellà, Can Xuec (BSPG 2003 $X X$ 6224). Middle Albian of Mexico (Sonora)

TABLE 33. Measurements of MGB 83310.

\begin{tabular}{ccccccc}
\hline & $\mathbf{n}$ & $\min -\max$ & $\boldsymbol{\mu}$ & $\mathbf{s}$ & $\mathbf{c v}$ & $\boldsymbol{\mu \pm s}$ \\
\hline clmin & 20 & $2.19-2.93$ & 2.55 & 0.21 & 8.3 & $2.34-2.76$ \\
clmax & 20 & $2.58-3.41$ & 2.91 & 0.25 & 8.7 & $2.66-3.17$ \\
ccd & 20 & $2.76-3.65$ & 3.22 & 0.27 & 8.4 & $2.94-3.49$ \\
$\mathrm{~s}$ & $6+6$ & & & & &
\end{tabular}



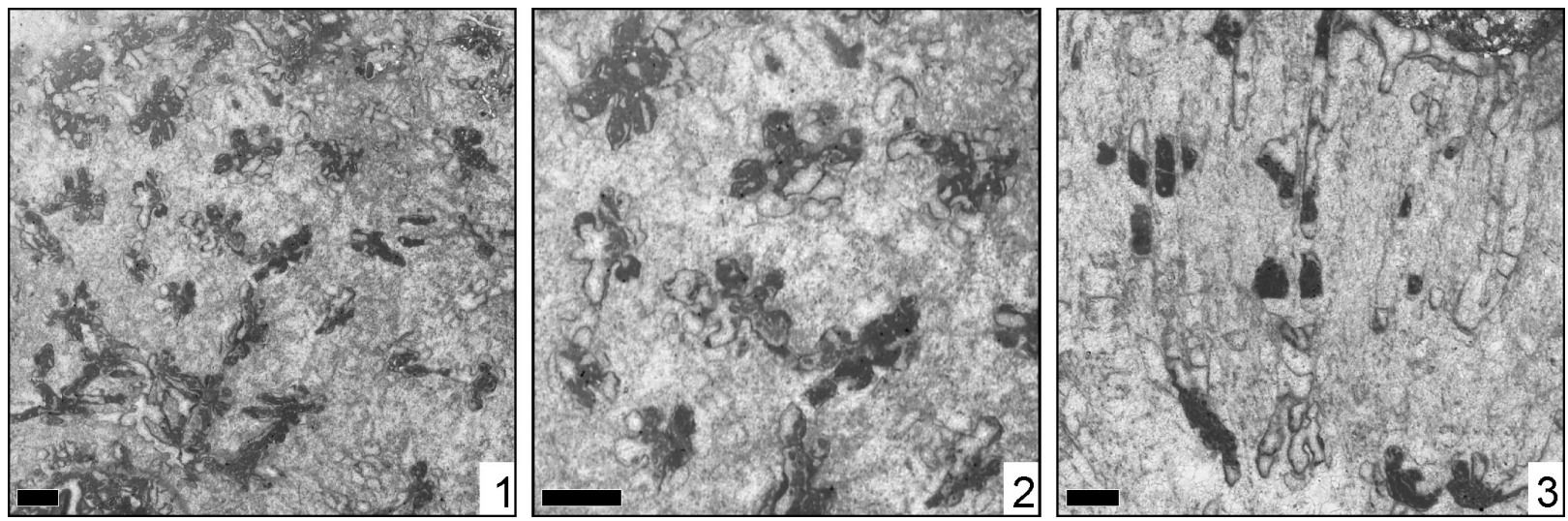

FIGURE 38. Solenocoenia gracilis Roniewicz, 1976, MGB 83379. 1: transversal thin section. 2: transversal thin section, detail. 3: longitudinal thin section. Scale $1 \mathrm{~mm}$.

Tepache, Lampazos area, Espinazo de Diablo (ERNO L-130103).

Genus SOLENOCOENIA Roniewicz, 1976

Type species. Convexastrea semiradiata Etallon, 1864 , by original designation.

Solenocoenia gracilis Roniewicz, 1976

Figure 38

V*1976 Solenocoenia gracilis Roniewicz, p. 114, pl. 14, fig. 4 ; pl. 15, fig. 1ab-2

Material. MGB 83379; 1 thin section.

Dimensions. See Table 34.

Remarks. The genus was recently described in detail (Löser, 2016). The present material compares well to Solenocoenia gracilis in its corallite dimensions and septal counts. The indication of the genus in the study area extends its range into the Lower Valanginian.

Occurrence. Upper Oxfordian to Lower Kimmeridgian of Romania (Tulcea) Dobrogea (BUFGG Do179). Kimmeridgian of Germany (Bayern) Saal an der Donau (FLH 1036). Lower Kimmeridgian of Romania (Tulcea) Topalu.
TABLE 34. Measurements of MGB 83379.

\begin{tabular}{ccccccc}
\hline & $\mathbf{n}$ & $\mathbf{m i n}-\mathbf{m a x}$ & $\boldsymbol{\mu}$ & $\mathbf{s}$ & $\mathbf{c v}$ & $\boldsymbol{\mu \pm \mathbf { s }}$ \\
\hline clmin & 15 & $0.74-1.22$ & 0.98 & 0.15 & 16.1 & $0.82-1.13$ \\
clmax & 15 & $0.81-1.73$ & 1.30 & 0.29 & 22.7 & $1.00-1.60$ \\
$\mathbf{s}$ & $6+6$ & & & & & \\
\hline
\end{tabular}

Superfamily HETEROCOENIOIDEA Oppenheim, 1930

Family CAROLASTRAEIDAE Eliášová, 1976 Genus COMALIA Wells, 1932

Type species. Comalia fasciculata Wells, 1932, by original designation.

Comalia fasciculata Wells, 1932

Figure 39

v*1932 Comalia fasciculata Wells, p. 255, pl. 30, figs. 11,11 a, pl. 37, fig. 2

v 2016 Comalia fasciculata Wells, 1932; Löser, fig. C42

Material. MGB 83329; 2 thin sections.

Dimensions. See Table 35.
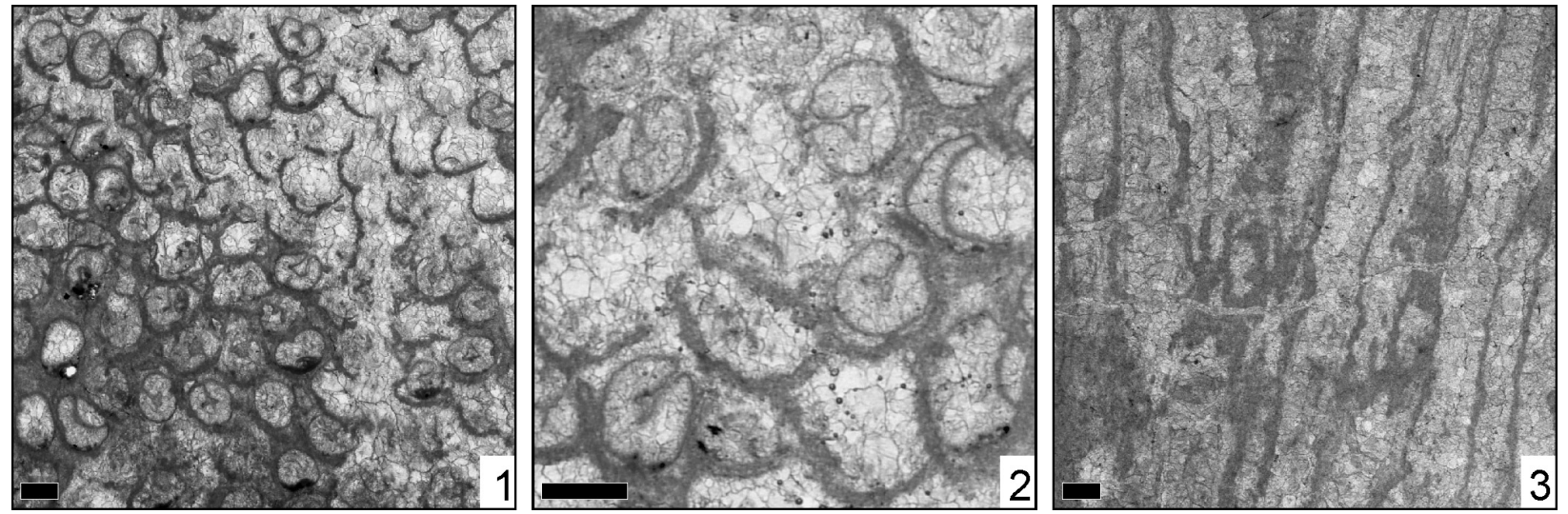

FIGURE 39. Comalia fasciculata Wells, 1932, MGB 83329. 1: transversal thin section. 2: transversal thin section, detail. 3: longitudinal thin section. Scale $1 \mathrm{~mm}$. 
TABLE 35. Measurements of MGB 83329.

\begin{tabular}{ccccccc}
\hline & $\mathbf{n}$ & $\mathbf{m i n}-\mathbf{m a x}$ & $\boldsymbol{\mu}$ & $\mathbf{s}$ & $\mathbf{c v}$ & $\boldsymbol{\mu \pm \mathbf { s }}$ \\
\hline clmin & 55 & $0.61-1.29$ & 0.94 & 0.12 & 13.4 & $0.81-1.06$ \\
clmax & 55 & $0.90-1.40$ & 1.12 & 0.11 & 10.6 & $1.00-1.24$ \\
ccd & 80 & $0.91-1.52$ & 1.25 & 0.13 & 11.1 & $1.11-1.39$ \\
\hline
\end{tabular}

Remarks. The genus was recently described and depicted (Löser, 2016). The genus is very similar to Pleurocoenia Orbigny, 1849, but differs by the formation of the inner margin of the only septum. The septum is without ornamentation in Pleurocoenia but branching in Comalia.

Occurrence. Callovian to Kimmeridgian of Japan (Kochi-ken) Takaoka-gun, Sakawa-cho, Kamo, Arinoki-dai near Umabara (TUM 43475-1). Tithonian to Lower Berriasian of Czech Republic (Moravia) Štramberk (CGS HF 215). Earlymost Albian (Tardefurcata Zone) of USA (Texas) Hays County, Blanco River, Pleasant Valley Crossing.

HOLOCOENIA group

Genus HOLOCOENIA Milne Edwards and Haime, 1851

Type species. Astrea micrantha Roemer, 1841, by original designation.

\section{Holocoenia cf. micrantha (Roemer, 1841)}

Figure 40

Material. MGB 83284; 2 thin sections.

Dimensions. See Table 36.

Remarks. The genus Holocoenia was recently revised (Löser, 2009) and again reported in Löser (2016). The present specimen differs from $H$. micrantha by a higher septal count. Most Holocoenia species have 20 septa and are only distinguished by the corallite dimensions.
TABLE 36. Measurements of MGB 83284.

\begin{tabular}{ccccccc}
\hline & $\mathbf{n}$ & $\mathbf{m i n}-\mathbf{m a x}$ & $\boldsymbol{\mu}$ & $\mathbf{s}$ & $\mathbf{c v}$ & $\boldsymbol{\mu \pm \mathbf { s }}$ \\
\hline clmin & 10 & $1.22-1.56$ & 1.34 & 0.12 & 9.4 & $1.21-1.46$ \\
$\operatorname{clmax}$ & 10 & $1.51-2.09$ & 1.72 & 0.17 & 10.1 & $1.55-1.90$ \\
$\operatorname{ccd}$ & 20 & $1.25-1.89$ & 1.62 & 0.20 & 12.8 & $1.41-1.83$ \\
$\mathrm{~s}$ & 10 & $21-26$ & 23.2 & 1.61 & 6.9 & $22-25$ \\
\hline
\end{tabular}

Superfamily MISISTELLOIDEA Eliášová, 1976

Family MISISTELLIDAE Eliášová, 1976

Genus MISCELLOSMILIA Eliášová, 1976

Type species. Miscellosmilia famosa Eliášová, 1976, by original designation.

Miscellosmilia sp.

Figure 41

Material. MGB 83264; 1 thin section.

Dimensions. (MGB 83264) c, $17 \mathrm{~mm}$; s, 10+10+20+40.

Remarks. The genus is very similar to Misistella but is solitary (not phaceloid) and has a stronger columella. The present specimen distinguishes from the type species by its decameral septal symmetry and probably represents a new species.

Occurrence. Tithonian to Lower Berriasian of Czech Republic (Moravia) Štramberk (CGS HF 636).

\section{Superfamily MONTLIVALTIOIDEA Felix, 1900 \\ Family MONTLIVALTIIDAE Felix, 1900 \\ Genus CLAUSASTREA Orbigny, 1849}

Type species. Clausastrea tessellata Orbigny, 1849, by monotypy.

\section{Clausastrea bolzei Alloiteau, 1960}

Figure 42

v 1960 Clausastraea bolzei Alloiteau, p. 23, textfigs. 8,9 , pl. 5 , figs. 2,4
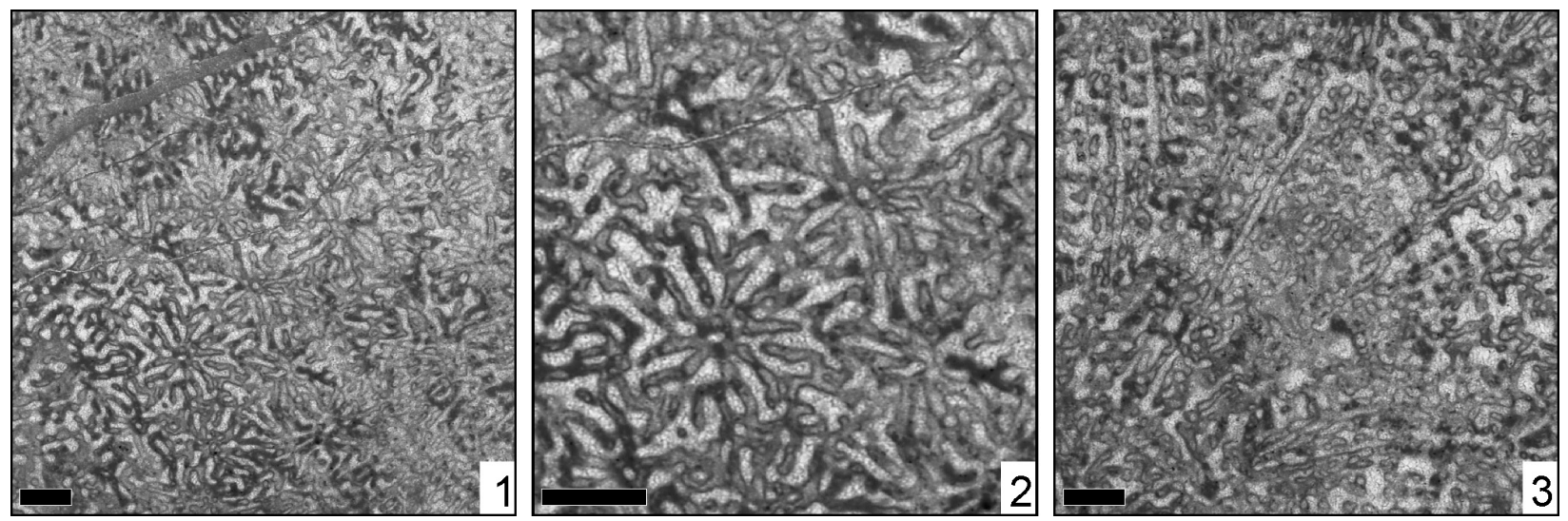

FIGURE 40. Holocoenia cf. micrantha Roemer, 1841, MGB 83284. 1: transversal thin section. 2: transversal thin section, detail. 3: longitudinal thin section. Scale $1 \mathrm{~mm}$. 

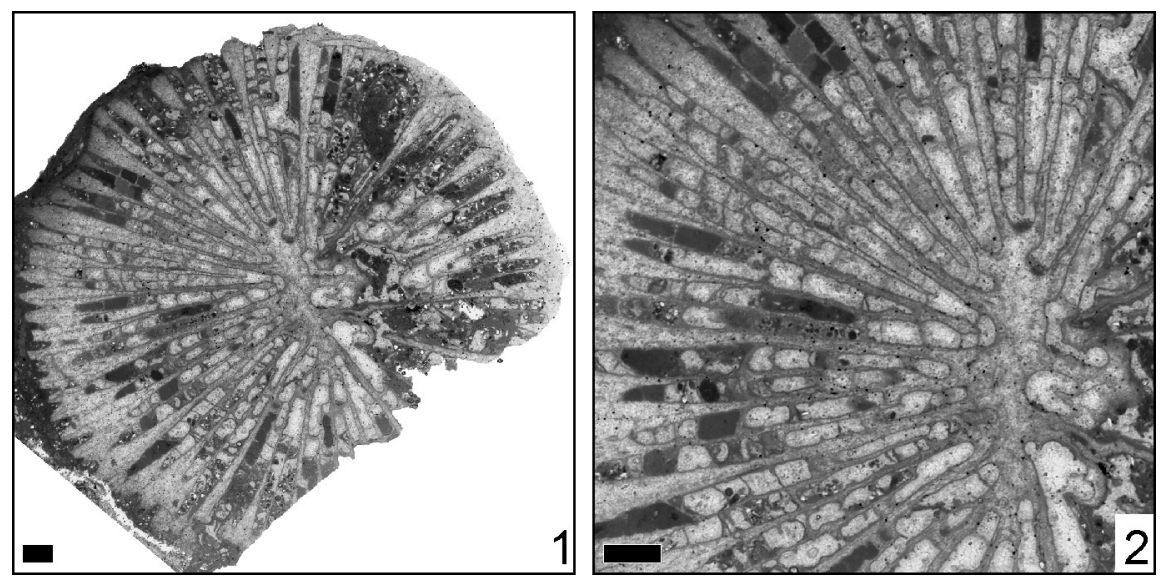

FIGURE 41. Miscellosmilia sp., MGB 83264. 1: transversal thin section. 2: transversal thin section, detail. Scale $1 \mathrm{~mm}$.

v $1960 \quad$ Clausastraea saltensis n.sp.; Alloiteau, p. 25, text-fig. 10, pl. 2, fig. 2

Material. MGB 83227; 2 thin sections.

Dimensions. See Table 37.

Remarks. The genus was described and depicted in Löser (2016). The present material differs from the diagnosis of the genus by confluent costae and corallites arranged in rows. Nevertheless, it compares well to the type material of $C$. bolzei.

Occurrence. Tithonian of Spain (Valencia, Alicante) La Querola (MNHN R10850). Tithonian to Lower Berriasian of Czech Republic (Moravia) Štramberk (CGS HF 3215). Valanginian to Aptian of Mexico (Puebla) San Juan Raya (IGM 9205). Lower Aptian (Tuarkyricus - Weissi Zone) of France (Vaucluse) Sault. Lower Aptian of Tunisia, Aïn el Baida, Cheid Mt.

Genus DIMORPHOCOENIA Fromentel, 1857

Type species. Dimorphastrea crassisepta Orbigny, 1850, by monotypy.
TABLE 37. Measurements of MGB 83227.

\begin{tabular}{ccccccc}
\hline & $\mathbf{n}$ & $\min -\mathbf{m a x}$ & $\boldsymbol{\mu}$ & $\mathbf{s}$ & $\mathbf{c v}$ & $\boldsymbol{\mu \pm \mathbf { s }}$ \\
\hline $\mathrm{crd}$ & 6 & $5.42-7.89$ & 6.56 & 0.91 & 13.8 & $5.65-7.47$ \\
$\mathrm{cdw}$ & 10 & $3.78-7.55$ & 5.39 & 1.10 & 20.4 & $4.28-6.49$ \\
$\mathrm{~s}$ & 10 & $15-20$ & 17.0 & 1.63 & 9.6 & $15-19$ \\
\hline
\end{tabular}

Dimorphocoenia multitabulata (Morycowa, 1971)

Figure 43

v*1971 Clausastraea alloiteaui multitabulata Morycowa, p. 83, pl. 18, fig. 2

Material. MGB 83328; 2 thin sections.

Dimensions. (MGB 83328) crd, 5.7-7 mm; cdw, 3.8-8.1 mm; s, 29-31.

Remarks. The genus was recently depicted and described (Löser, 2016). The present specimen has less septa than the type of Dimorphocoenia multitabulata (30-40).

Occurrence. Lower Aptian (Tuarkyricus - Weissi Zone) of France (Vaucluse) Sault (FSL). Lower Aptian (Lenticularis Zone) of Romania (Suceava)
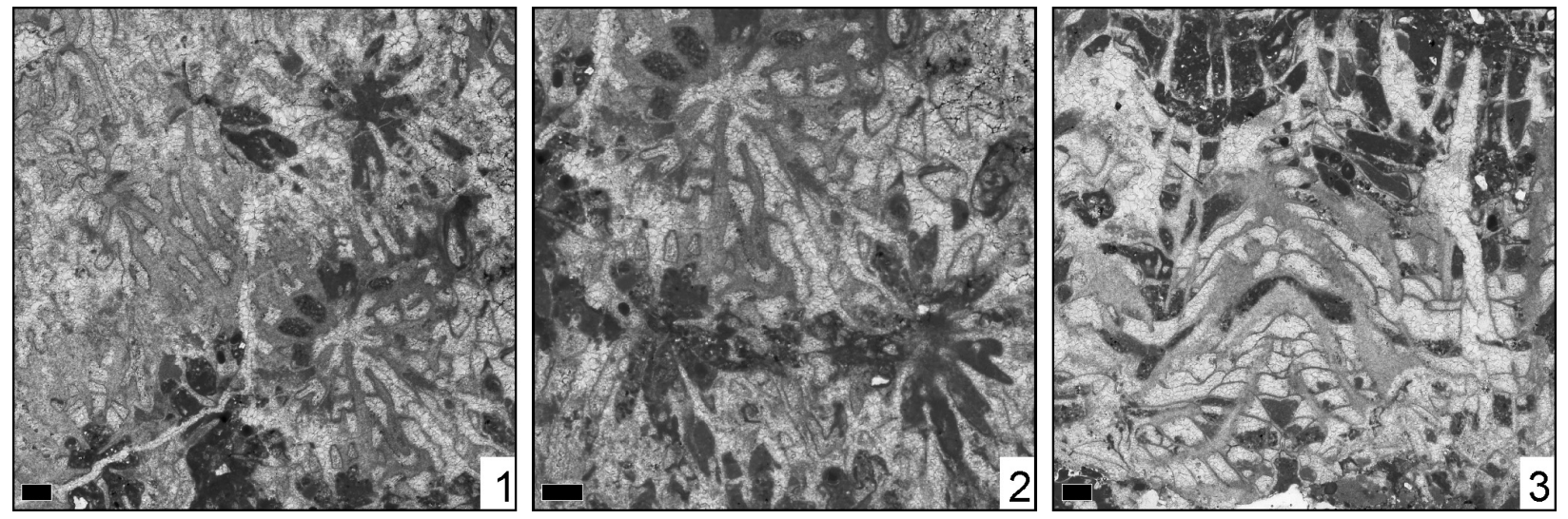

FIGURE 42. Clausastrea bolzei Alloiteau, 1960, MGB 83227. 1: transversal thin section. 2: transversal thin section, detail. 3: longitudinal thin section. Scale $1 \mathrm{~mm}$. 

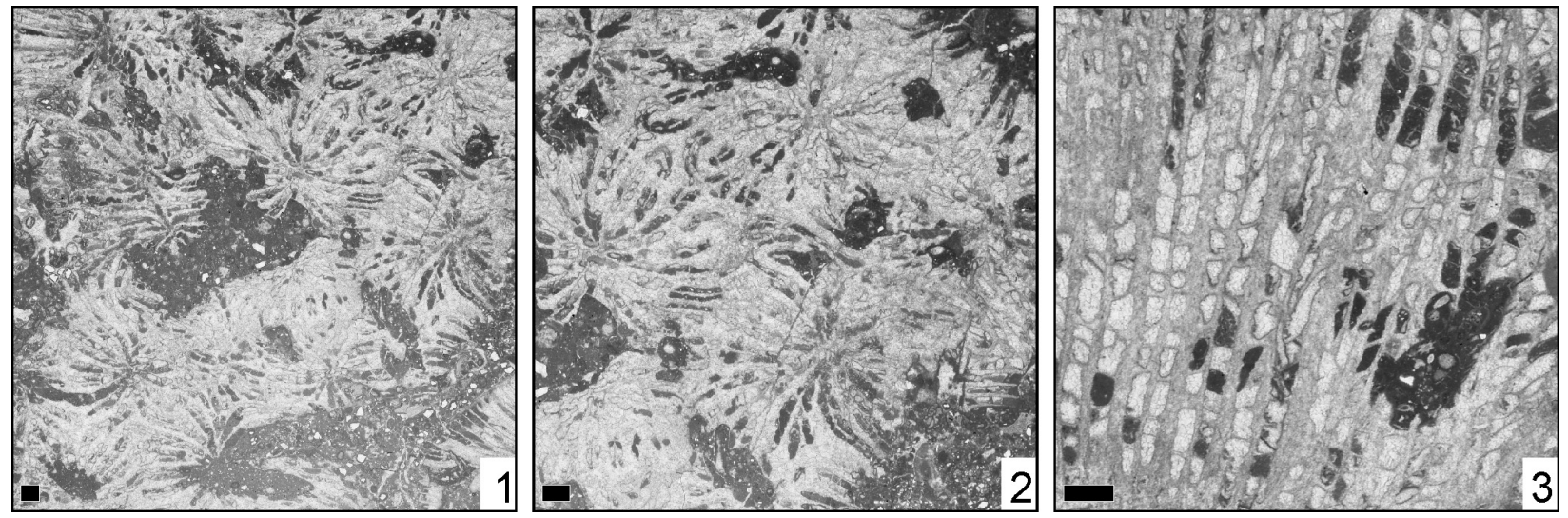

FIGURE 43. Dimorphocoenia multitabulata (Morycowa, 1971), MGB 83328. 1: transversal thin section. 2: transversal thin section, detail. 3: longitudinal thin section. Scale $1 \mathrm{~mm}$.

Pojorîta area, Cîmpulung-Moldovenesc, Valea Izvorul Alb.

Superfamily STYLINOIDEA Orbigny, 1851 Family AULASTRAEOPORIDAE Alloiteau, 1957 Genus EOPREVERASTREA gen. nov. Löser zoobank.org/5C7B540D-5D39-48EF-9C35-A42430602B37

Type species. Eopreverastrea llanoensis sp. nov. Löser.

Etymology. As a possible precursor of the genus Preverastraea.

Diagnosis. Astreoid colony. Corallite outline irregular. Septa compact. Microstructure of small-sized trabeculae, septa with a central dark line. Septa in cross section externally slightly thicker, tapering slightly towards the centre. Septal maximum thickness $500 \mu \mathrm{m}$. Symmetry of septa radial and regularly developed, in the type species in a pentameral symmetry. Cycles of septa subregular, one cycle of five septa in the type species. Septa hardly enter into the calicular space, they are very short, reduced to ridges, not connected to each other. A main septum is present but it is very thin and rather an apophysal ornamentation. Septal distal margin unknown, lateral face with fine thorns, inner margin with apophysal septa. All septa visible in the corallite are apophysal septa. They are thin and in an irregular number. Pali absent. Costae present, confluent or not. Synapticulae absent. Columella absent. Endotheca consists of large dissepiments. The compact wall has the same structure as septa, is therefore septothecal. Coenosteum broad, with the same extend as the corallite diameter, consists of costae and exothecal dissepiments. Budding extracalicinal.

Comparison. Closely related are Preverastrea Beauvais, 1976, and Paracanthogyra Morycowa and Marcopoulou-Diacantoni, 1997. In both gen- era, septa enter in the corallite space, and long apophysal septa are absent.

Species. Only the type species.

Eopreverastrea llanoensis sp. nov. Löser Figures 44, 45

zoobank.org/E040CD3D-022A-42BD-9035-B3B46C3446C6

Etymology. After the locality Llano.

Holotype. MGB 83265 with three thin sections.

Type locality. Puerto Llano section, Cabañas, Sierra de Cazorla, Jaén, Andalucía, Spain.

Type level. Sierra del Pozo Fm, Lower Valanginian.

Depository. Museo de Geología de Barcelona, Spain.

Diagnosis. Eopreverastrea with small corallites (1$2 \mathrm{~mm}$ ), five septa and between 2 and 12 apophysal septa, of which one is longer than all other.

Material. MGB 83265; 3 thin sections.

Dimensions. See Table 38.

Description. As for the genus.

Family RHIPIDOGYRIDAE Koby, 1905

Genus PLACOGYRA Koby, 1905

Type species. Placogyra felixi Koby, 1905, by monotypy.

\section{Placogyra cf. hykeli Eliášová, 1973}

Figure 46

v2008 Ogilvinella sp.; Tomás, Löser and Salas Roig, p. 525, fig. 13I, J

Material. MGB 83345; 1 thin section.

Dimensions. See Table 39.

Remarks. The genus was just recently described and depicted (Löser, 2016). The present material has larger dimensions than Placogyra hykeli.

Occurrence. Upper Aptian of Spain (Valencia, Castellón) Benicasin, La Venta. 


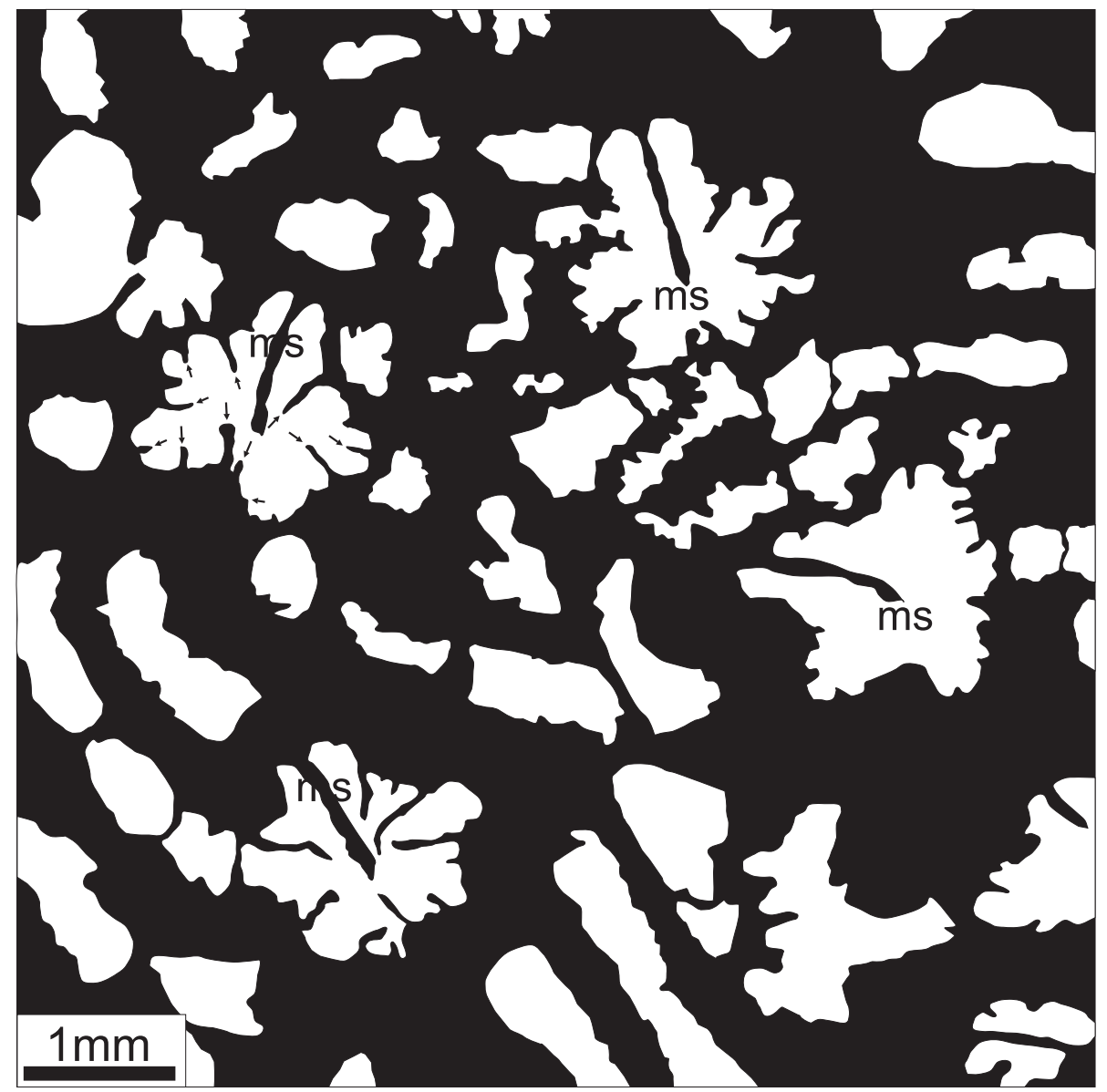

FIGURE 44. Eopreverastrea llanoensis gen.nov. sp. nov., Löser. Drawing after MGB 83265. Ms, main septum. Arrows point to apophysal septa. Scale $1 \mathrm{~mm}$.

Genus RHIPIDOGYRA Milne Edwards and Haime, 1848

Type species. Lobophyllia flabellum Michelin, 1843 , by subsequente definition.

\section{Rhipidogyra sp. \\ Figure 47}

Material. MGB 83291, 83299, 83308; 3 thin sections.

Dimensions. (MGB 83299) c, $11.8 \times 64.4 \mathrm{~mm}$; s, 350; sd 15 / 5 mm.

Remarks. Rhipidogyra is a mostly Jurassic genus; the majority of Cretaceous species assigned to this genus belong to other genera. More than 20 species are described from the Jurassic. Because the knowledge is poor how these species have to be distinguished, the present material remains in open nomenclature.

Family STYLINIDAE Orbigny, 1851 Genus ALLOITEAUCOENIA Beauvais, 1964
Type species. Alloiteaucoenia tumularis Beauvais, 1964, by original designation.

Alloiteaucoenia bernardina (Orbigny, 1850)

Figure 48

Material. MGB 83229, 83304; 6 thin sections.

$\mathrm{V}^{*} 1850 \quad$ Pseudocoenia bernardina Orbigny, p. 34

v 1979 Heliocoenia variabilis Etallon, 1859; Sikharulidze, p. 10, text-fig. 3 , pl. 1 , fig. 1 , pl. 4 , fig. 1

v 2016 Pseudocoenia bernardina Orbigny, 1850; Löser, fig. P125

2018 Heliocoenia variabilis Etallon, 1859; Ricci, Lathuilière and Rusciadelli, p. 469 pl. 16, fig. $1-4$

Dimensions. See Table 40.

Remarks. Pseudocoenia was originally established by Orbigny (1850) as a "Cryptocoenia with eight systems", which means a Cryptocoenia with a basic septal symmetry of eight. Wells (1936) designated the type species and selected a lectotype. This lectotype does not correspond to the original description of Orbigny. An attempt to correct this 

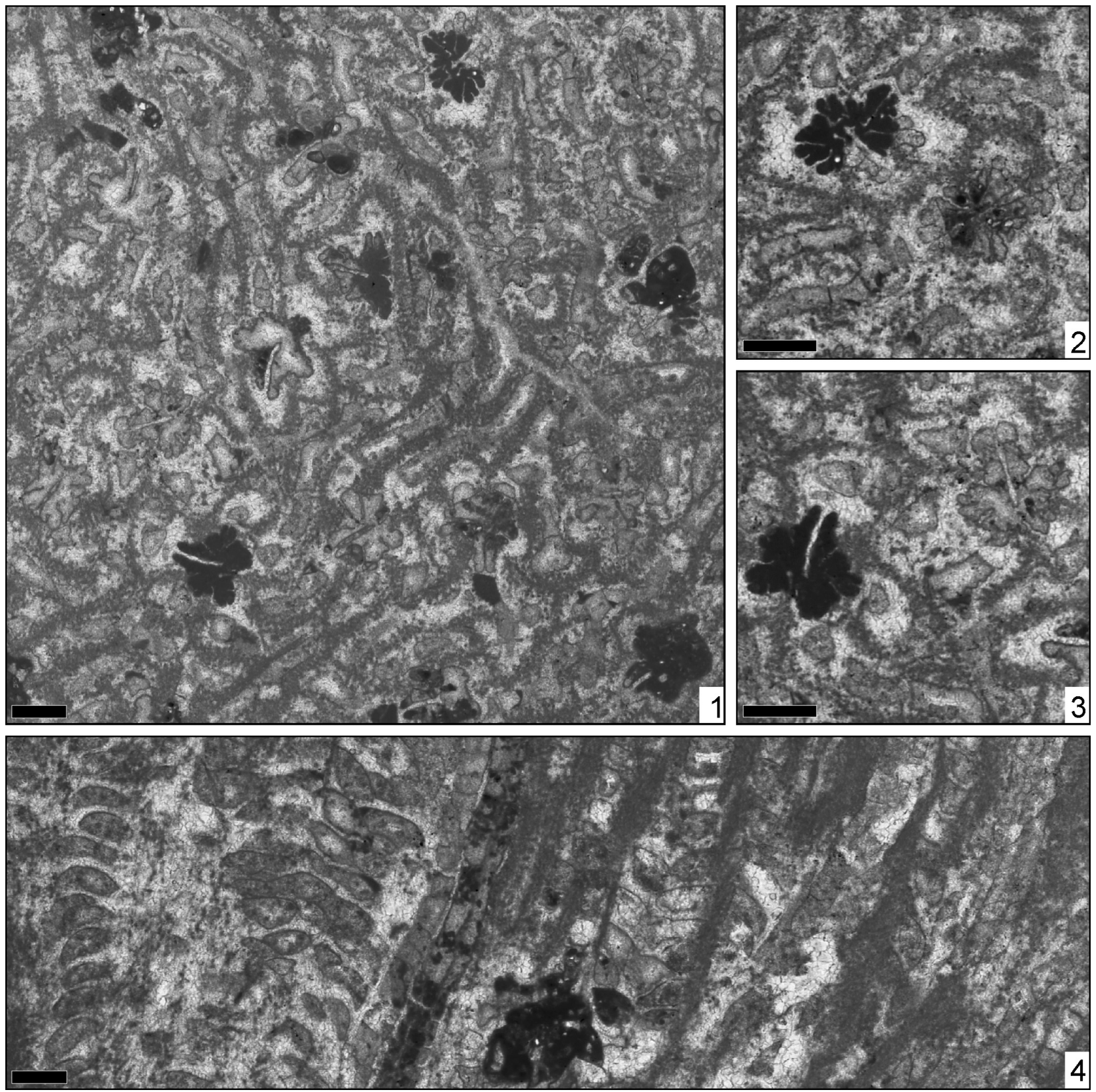

FIGURE 45. Eopreverastrea llanoensis gen.nov. sp. nov., Löser, MGB 83265. 1: transversal thin section. 2: transversal thin section, detail. 3: transversal thin section, detail. 4: longitudinal thin section. Scale $1 \mathrm{~mm}$.

TABLE 38. Measurements of MGB 83265.

\begin{tabular}{ccccccc}
\hline & $\mathbf{n}$ & $\mathbf{m i n}-\mathbf{m a x}$ & $\boldsymbol{\mu}$ & $\mathbf{s}$ & $\mathbf{c v}$ & $\boldsymbol{\mu \pm \mathbf { s }}$ \\
\hline $\operatorname{clmin}$ & 30 & $0.97-1.75$ & 1.45 & 0.19 & 13.4 & $1.25-1.64$ \\
$\operatorname{clmax}$ & 30 & $1.02-1.93$ & 1.52 & 0.20 & 13.7 & $1.31-1.73$ \\
$\operatorname{ccd}$ & 30 & $2.01-3.59$ & 2.76 & 0.43 & 15.9 & $2.32-3.20$ \\
$\mathrm{~s}$ & 10 & $7-17$ & 11.8 & 3.55 & 30.1 & $8-15$
\end{tabular}

mistake by a decision of the International Commission on Zoological Nomenclature failed (Löser, 2007; Anonymous, 2013). The above mentioned lectotype (figured in Löser, 2016) shows characteristics that were later assigned to Heliocoenia EtalIon, 1859 and Alloiteaucoenia. In order to avoid further confusion, we discard Pseudocoenia and prefere to use Alloiteaucoenia. The type material of its type species is available and was recently depicted (Löser, 2016). The type material of the type species of Heliocoenia is not available, and 

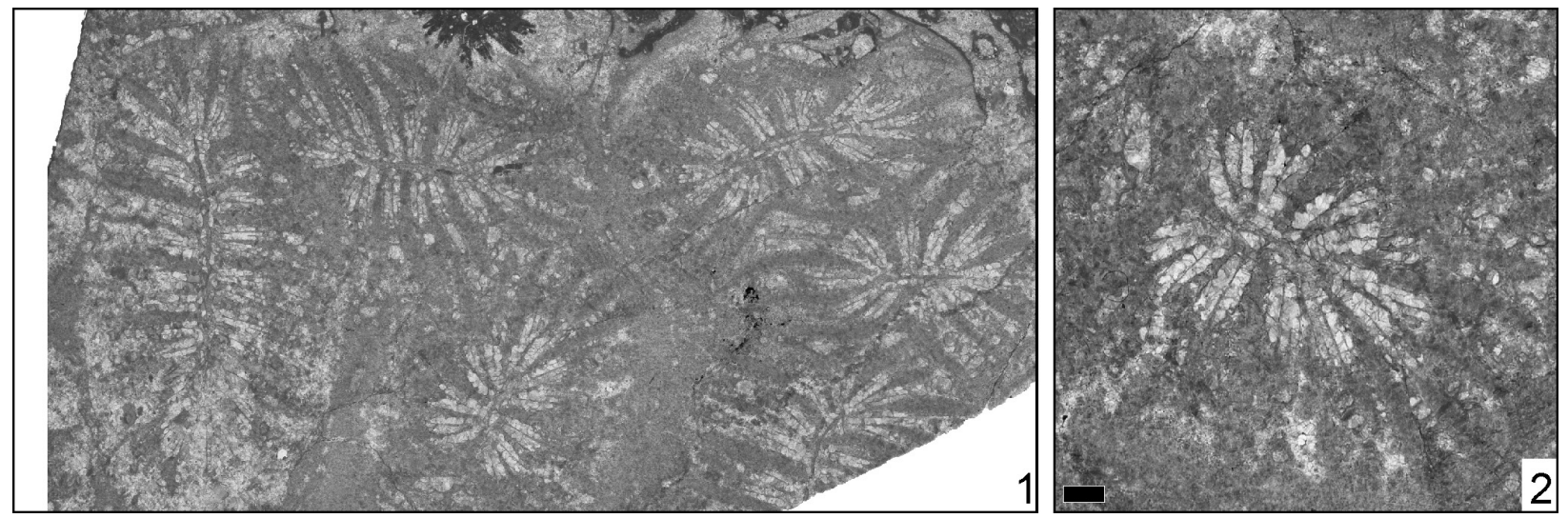

FIGURE 46. Placogyra cf. hykeli Eliášová, 1973, MGB 83345. 1: transversal thin section. 2: transversal thin section, detail. Scale $1 \mathrm{~mm}$.

TABLE 39. Measurements of MGB 83345.

\begin{tabular}{ccccccc}
\hline & $\mathbf{n}$ & $\boldsymbol{m i n}-\mathbf{m a x}$ & $\boldsymbol{\mu}$ & $\mathbf{s}$ & $\mathbf{c v}$ & $\boldsymbol{\mu \pm s}$ \\
\hline clmin & 5 & $4.84-8.00$ & 6.42 & 1.49 & 23.2 & $4.92-7.91$ \\
clmax & 4 & $9.92-17.2$ & 12.5 & 3.22 & 25.8 & $9.25-15.7$ \\
$\mathrm{~s}$ & 4 & $37-52$ & 44.8 & 8.38 & 18.7 & $36-53$ \\
\hline
\end{tabular}

the illustration in Etallon (1859) gives no information.

Occurrence. Oxfordian of France (Ain) Nantua, Landeyron (MNHN R09199); Charix (MJSN S2247). Kimmeridgian to Tithonian of Italy (Abruzzi, L'Aquila) Scanno, Monte Rotondo. Tithonian to Berriasian of Georgia (Racha) Tskhanari. Upper Tithonian of Austria (Oberösterreich) Ewigen Wand N Bad Goisern (HJGL nn).

Genus STYLINA de Lamarck, 1816

Type species. Stylina echinulata de Lamarck, 1816, by monotypy.

Remarks. The taxonomic problems of the genus Stylina were discussed in Löser (2016). The genus is conceptual, but widely accepted in the sense of Gill (1977) as a plocoid colony with septa that bear at their septal inner margins small ear-like ornamentations, named by Gill auriculae. For the distinction of the various Stylina species in the study area see Table 41.

\section{Stylina arborea Achiardi, 1880,}

Figure 49

v*1880 Stylina arborea Achiardi, p. 290, pl. 19, fig. 8 1985 Stylina strambergensis Geyer, 1955; Geyer and Rosendahl, p. 167, pl. 2, fig. 8

v 2013 Stylina inwaldiensis (Ogilvie, 1897); Löser, Werner, and Darga, p. 66, pl. 9, figs. 10-12

v 2016 Plesiostylina hourcqi Alloiteau, 1958; Löser, fig. P81

Material. MGB 83384; 1 thin section.

Dimensions. See Table 42.

Occurrence. Middle Jurassic of Spain (Andalucía, Cordoba) Puente Genil (UJDE P44); Spain (Andalucia, Sevilla) Estepa (UJDE M7545). Bathonian of Madagascar, Ampandrabé (MNHN M05152). Kimmeridgian of Germany (Bayern) Saal an der Donau

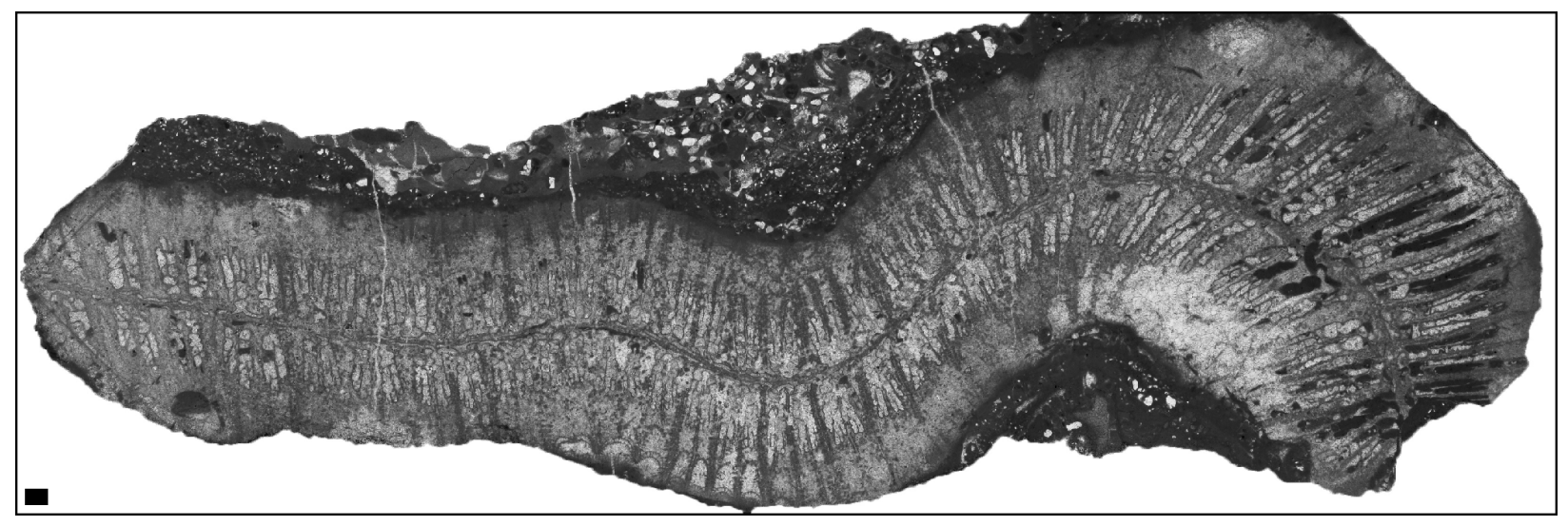

FIGURE 47. Rhipidogyra sp., MGB 83299. transversal thin section. Scale $1 \mathrm{~mm}$. 

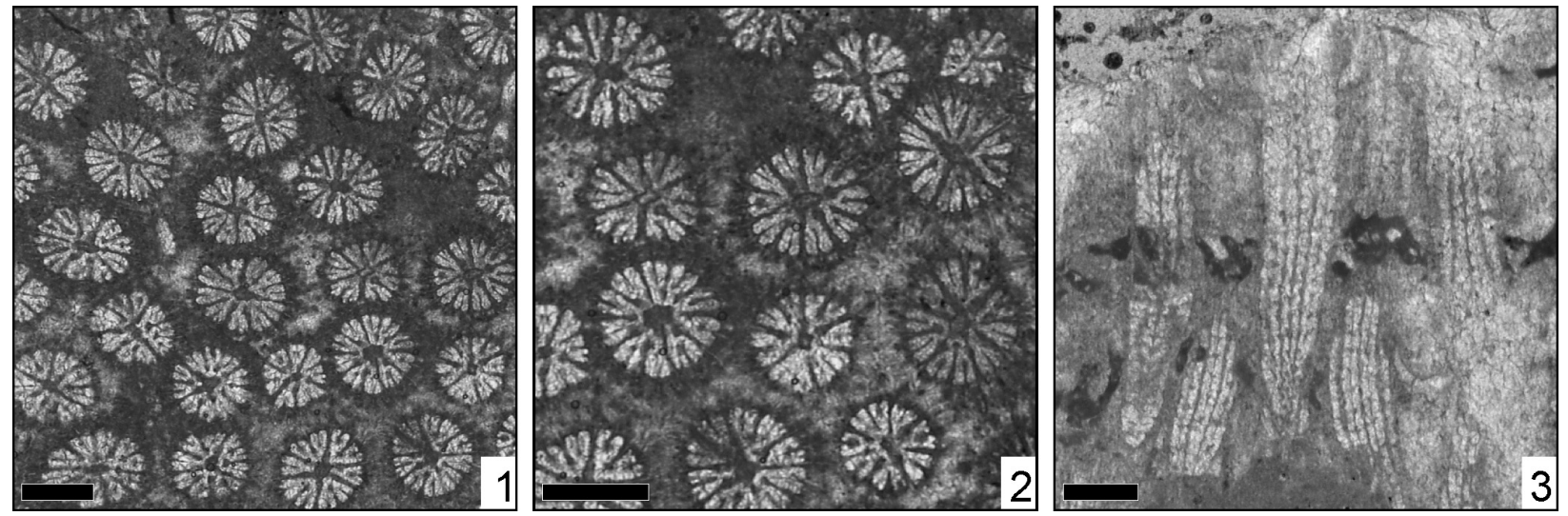

FIGURE 48. Alloiteaucoenia bernardina (Orbigny, 1850), MGB 83304. 1: transversal thin section. 2: transversal thin section, detail. 3: Iongitudinal thin section. Scale $1 \mathrm{~mm}$.

TABLE 40. Measurements of MGB 83304.

\begin{tabular}{ccccccc}
\hline & $\mathbf{n}$ & $\min -\mathbf{m a x}$ & $\boldsymbol{\mu}$ & $\mathbf{s}$ & $\mathbf{c v}$ & $\boldsymbol{\mu \pm \mathbf { s }}$ \\
\hline clmin & 40 & $0.84-1.10$ & 0.97 & 0.07 & 7.3 & $0.90-1.04$ \\
$\operatorname{clmax}$ & 40 & $0.99-1.28$ & 1.14 & 0.06 & 5.2 & $1.08-1.20$ \\
$\operatorname{ccd}$ & 40 & $1.11-1.59$ & 1.33 & 0.12 & 9.6 & $1.20-1.46$ \\
$\mathrm{~s}$ & 10 & $20-24$ & 21.8 & 1.68 & 7.7 & $20-23$ \\
\hline
\end{tabular}

TABLE 41. Distinction of the Stylina species within the study area.

\begin{tabular}{cccl}
\hline $\begin{array}{c}\text { Septal } \\
\text { symmetry }\end{array}$ & $\begin{array}{c}\text { Septal } \\
\text { cycles }\end{array}$ & $\begin{array}{c}\text { Small lumen } \\
(\mathbf{m m})\end{array}$ & \multicolumn{1}{c}{ Species } \\
\hline hexameral & 2 & $1.4-1.7$ & St. digitiformis \\
& & $1.9-2.1$ & St. sp. 1 \\
& 3 & $1.7-2.0$ & St. inflata \\
& 4 & $2.1-2.6$ & St. sp. 2 \\
octameral & 2 & $1.0-1.2$ & St. arborea \\
& & $1.2-1.5$ & St. lamellosa \\
decameral & 2 & $0.8-0.9$ & St. sp. 3 \\
& & $1.1-1.3$ & St. sp. 4 \\
\hline
\end{tabular}

TABLE 42. Measurements of MGB 83384

\begin{tabular}{ccccccc}
\hline & $\mathbf{n}$ & min-max & $\boldsymbol{\mu}$ & $\mathbf{s}$ & $\mathbf{c v}$ & $\boldsymbol{\mu \pm s}$ \\
\hline $\operatorname{clmin}$ & 6 & $1.00-1.28$ & 1.13 & 0.10 & 9.0 & $1.03-1.24$ \\
$\operatorname{clmax}$ & 6 & $1.25-1.54$ & 1.41 & 0.10 & 7.1 & $1.31-1.51$ \\
$\operatorname{ccd}$ & 10 & $1.24-1.79$ & 1.51 & 0.20 & 13.6 & $1.30-1.71$ \\
$\mathrm{~s}$ & $8+8$ & & & & &
\end{tabular}

(FLH 1046). Lower Tithonian of Italy (Veneto, Friuli) Monte Cavallo, Polcenigo, Coltura di Sotto. Middle Cenomanian of Germany (Bayern) RoßsteinAlmen.

Stylina digitiformis Achiardi, 1880

Figure 50

v 1880 Stylina digitiformis Achiardi, p. 302, pl. 20, fig. 6

1993 Stylina regularis de Fromentel, 1862; Morycowa and Decrouez, p. 204, pl. 1, fig. 2

Material. MGB 83355; 1 thin section.

Dimensions. See Table 43.
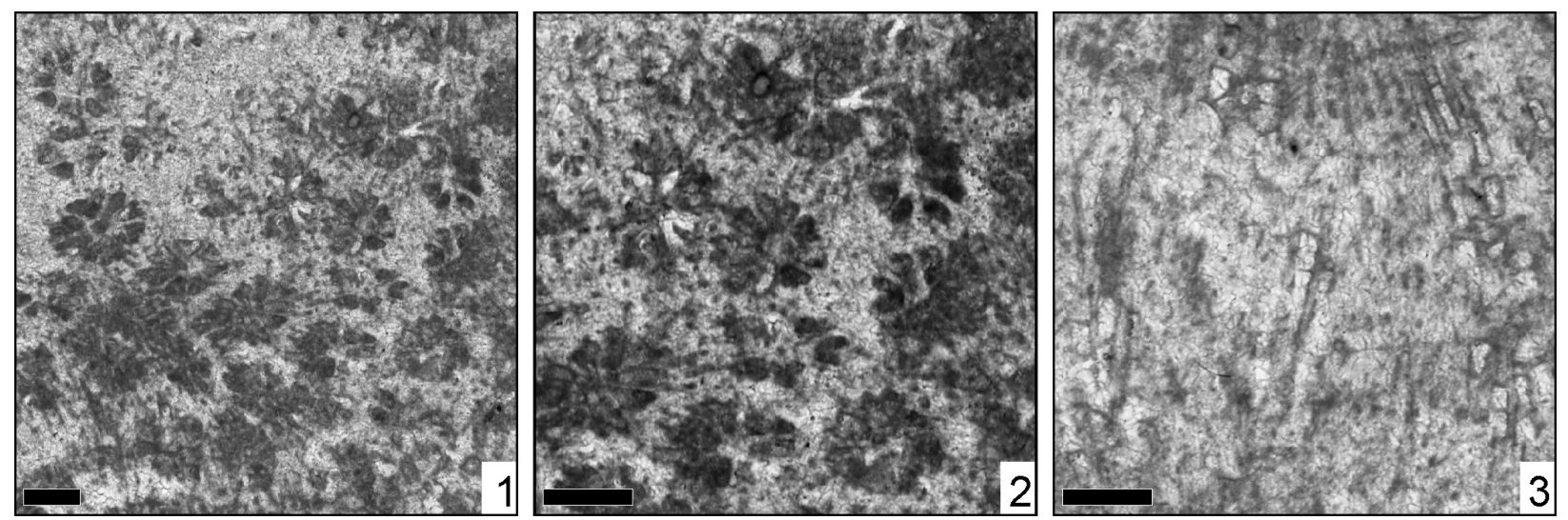

FIGURE 49. Stylina arborea Achiardi, 1880, MGB 83384. 1: transversal thin section. 2: transversal thin section, detail. 3: longitudinal thin section. Scale $1 \mathrm{~mm}$. 

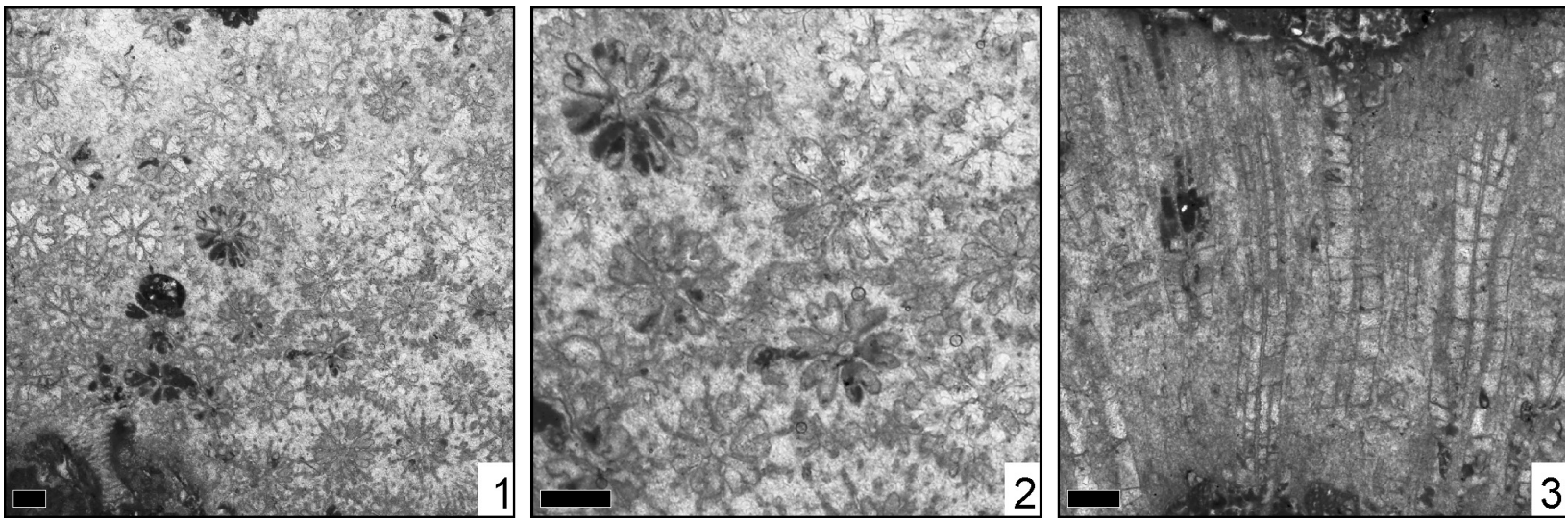

FIGURE 50. Stylina digitiformis Achiardi, 1880, MGB 83355. 1: transversal thin section. 2: transversal thin section, detail. 3: longitudinal thin section. Scale $1 \mathrm{~mm}$.

TABLE 43. Measurements of MGB 83355.

\begin{tabular}{ccccccc}
\hline & $\mathbf{n}$ & $\min -\mathbf{m a x}$ & $\boldsymbol{\mu}$ & $\mathbf{s}$ & $\mathbf{c v}$ & $\boldsymbol{\mu \pm s}$ \\
\hline clmin & 20 & $1.35-1.72$ & 1.52 & 0.12 & 8.3 & $1.39-1.65$ \\
$\operatorname{clmax}$ & 20 & $1.40-1.98$ & 1.64 & 0.16 & 10.3 & $1.47-1.81$ \\
$\operatorname{ccd}$ & 20 & $1.46-2.76$ & 2.14 & 0.37 & 17.3 & $1.77-2.51$ \\
$\mathrm{~s}$ & $6+6$ & & & & & \\
\hline
\end{tabular}

Occurrence. Lower Tithonian of Italy (Veneto, Friuli) Monte Cavallo, Polcenigo, Coltura di Sotto. Upper Tithonian to Berriasian of France (Var) Grasse, St. Vallier-de-Thiey, Col de Ferrier (SGM Fer. 16). Middle Berriasian of France (HauteSavoie) Mont Salève (MB 2817). Lower Hauterivian of France (Bouches-du-Rhône) Marseille, Calanque de la Mounine (BSPG 2003 XX 5188). Lower Barremian of France (Haute-Savoie) Bornes, Pointe Blanche.

Stylina inflata Fromentel, 1856 Figure 51

$\mathrm{v}^{*} 1856 \quad$ Stylina inflata Fromentel, p. 857 v 1863a Stylina inflata; Fromentel, p. 30, pl. 3, fig. 1 v 1875 Stylina spissa; Becker, p. 147, pl. 37, fig. 4 v 1881 Diplocoenia polymorpha; Koby, p. 72, pl. 18, fig. 3 pl. 19, fig. 1

v 1881 Stylina subramosa; Koby, p. 79, pl. 15, fig. 3

v 1909b Heliocoenia humberti Etallon; Prever, p. 994 fig. 9

$1964 \quad H e l i o c o e n i a$ carpathica n.sp.; Morycowa, p. 42 , text-fig. 5 , pl. 6 , fig. 3 , pl. 7 , figs. 4,5 , pl. 8 , figs. 1,2 , pl. 11 , fig. 3

1985 Stylina sablensis Trautschold, 1886; Sikharulidze, p. 16, pl. 2, fig. 3

v1994 Diplocoenia hayasakai Eguchi; Liao and Xia, p. 176, pl. 52, figs. 9, 10

v 1995 Stylina cf. sparsa Trautschold 1886; Löser and Raeder, p. 43

v1997 Heliocoenia carpathica Morycowa, 1964; Baron-Szabo, p. 37, pl. 2, fig. 4

v 2006 Stylina carpathica (Morycowa, 1964); Löser and Ferry, p. 476, figs. 3.6, 3.8

v 2014 Heliocoenia carpathica Morycowa, 1964; Baron-Szabo, pl. 81, fig. 6
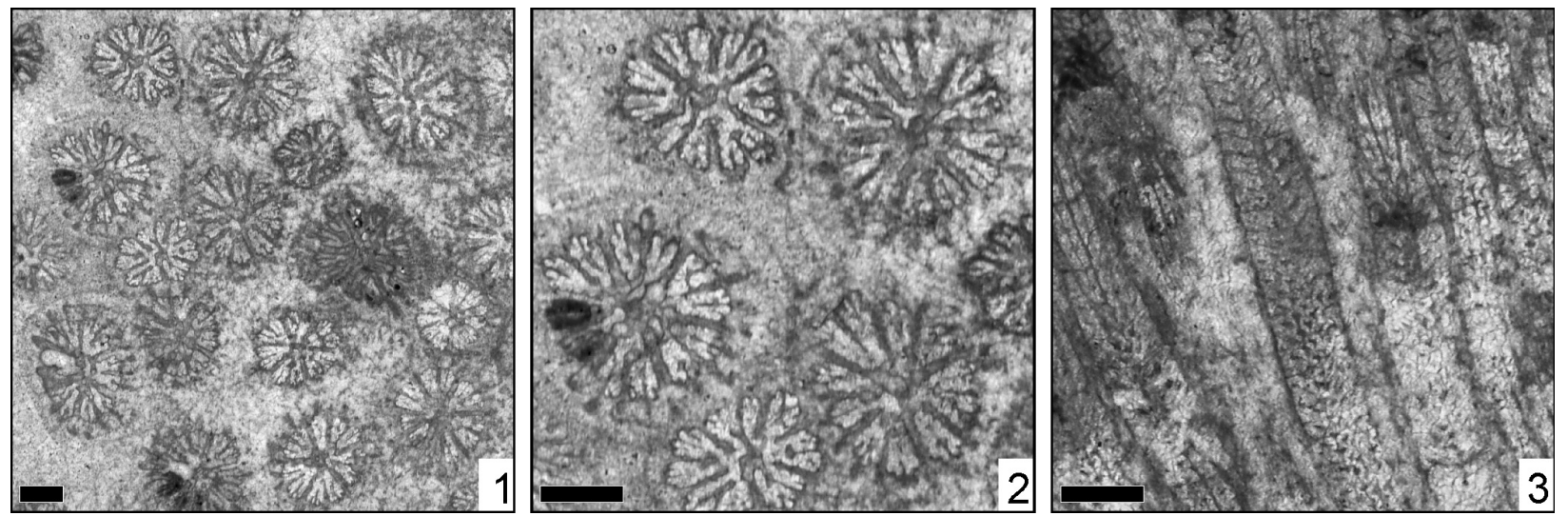

FIGURE 51. Stylina inflata Fromentel, 1856, MGB 83258. 1: transversal thin section. 2: transversal thin section, detail. 3: longitudinal thin section. Scale $1 \mathrm{~mm}$. 
TABLE 44. Measurements of MGB 83258.

\begin{tabular}{ccccccc}
\hline & $\mathbf{n}$ & min-max & $\boldsymbol{\mu}$ & $\mathbf{s}$ & $\mathbf{c v}$ & $\boldsymbol{\mu \pm \mathbf { s }}$ \\
\hline clmin & 35 & $1.64-2.05$ & 1.84 & 0.11 & 6.4 & $1.72-1.96$ \\
clmax & 35 & $1.90-2.63$ & 2.23 & 0.16 & 7.5 & $2.06-2.40$ \\
ccd & 30 & $2.16-3.38$ & 2.72 & 0.33 & 12.1 & $2.39-3.05$ \\
s & $6+6+12$ & & & & & \\
\hline
\end{tabular}

Material. MGB 83258, 83292, 83296, 83300, $83327,83333,83341 ; 3$ thin sections.

Dimensions. See Table 44.

Occurrence. Bathonian of Madagascar, Aontzy (MNHN M05181). Oxfordian of Switzerland (Jura) Berner Jura. Upper Oxfordian of Poland (Swietokrzyskie) Sainte-Croix, Niziny (ZPAL Hiii807). Kimmeridgian (Beckeri Zone, Ulmense Subzone) of Germany (Baden-Württemberg) Nattheim. Kimmeridgian to Tithonian of Japan (Oita-ken) Minamiamabe-gun, Honjo-mura, Oyabu valley $1.5 \mathrm{~km} \mathrm{SW}$ Shinkai (NSM PA 12551). Tithonian of Spain (Iberian Chaines) Mezalocha (RUB ME163); France (Haute-Saône) Gray, Mantoche (MNHN M03646); Italy (Abruzzi, L'Aquila) Paganica, Assergi-Portella. Berriasian to Valanginian of China (Xizang [= Tibet] Autonomous Region) Baingoin county, Toiba district, Qangma, Riabadange. Berriasian to Hauterivian of France (Gard) Allègre (MHNG 4688). Hauterivian of Georgia (Imereti) Mokhorotubani. Barremian of France (Doubs) Morteau (MHNN 26778); France (Drôme) Remuzat, Valley of Arnayon (SGM Arn. 11). Lower Barremian of France (Drôme) Vercors Mts, section NW Archiane. Upper Barremian of France (Ardèche) St.Remèze, Pont de Laval. Upper Barremian to Lower Aptian (Sartousi - Weissi Zone) of Germany (Bayern) Allgäuer Helvetikum, Gottesackerloch. Lower Aptian (Tuarkyricus - Weissi Zone) of France (Vaucluse) Vaucluse Mts, Rustrel (UP 110).
Lower Aptian of Greece (Viotía) Arachova (BSPG 2003 XX 5543); Levadia, Perachorion; Arachova (BSPG 2003 XX 5573); Levadia, Perachorion; Poland (Malopolskie, Wadowice) Lanckorona, Jastrzebia. Lower Aptian (Lenticularis Zone) of Greece (Viotía) Levadia, roadcut near Perachorion NW Levadia (BSPG 2003 XX 5736).

\section{Stylina ? lamellosa Trautschold, 1886}

Figure 52

* 1886 Stylina lamellosa Trautschold, p. 125, pl. 3, figs. 4 a-c

Material. MGB 83255, 83256, 83289, 83290; 3 thin sections.

Dimensions. See Table 45.

Remarks. The type material of the species was not available for study. Trautschold (1886) mentioned that the species has only eight septa, whereas the present material has 16 septa. No octameral Stylina with only eight septa is known, so the material of Trautschold was probably just poorly preserved.

Occurrence. Kimmeridgian (Beckeri Zone, Ulmense Subzone) of Germany (Baden-Württemberg) Gerstetten, Neubaugebiet Süd, Komplex V (SMNS nn). Kimmeridgian of Germany (Bayern) Saal an der Donau (FLH 4450). Tithonian to Lower Berriasian of Czech Republic (Moravia) Štramberk (CGS HF 2967). Lower Valanginian of France (Bouches-du-Rhône) Marseille, Butte de L'Escalette, onshore sample locality (Feld). Lower

TABLE 45. Measurements of MGB 83255.

\begin{tabular}{ccccccc}
\hline & $\mathbf{n}$ & $\mathbf{m i n}-\mathbf{m a x}$ & $\boldsymbol{\mu}$ & $\mathbf{s}$ & $\mathbf{c v}$ & $\boldsymbol{\mu \pm \mathbf { s }}$ \\
\hline clmin & 30 & $1.06-1.59$ & 1.34 & 0.15 & 11.5 & $1.18-1.49$ \\
$\operatorname{clmax}$ & 30 & $1.25-1.77$ & 1.51 & 0.14 & 9.8 & $1.36-1.66$ \\
$\operatorname{ccd}$ & 30 & $1.43-2.33$ & 1.87 & 0.25 & 13.7 & $1.61-2.12$ \\
$\mathrm{~s}$ & $8+8$ & & & & & \\
\hline
\end{tabular}
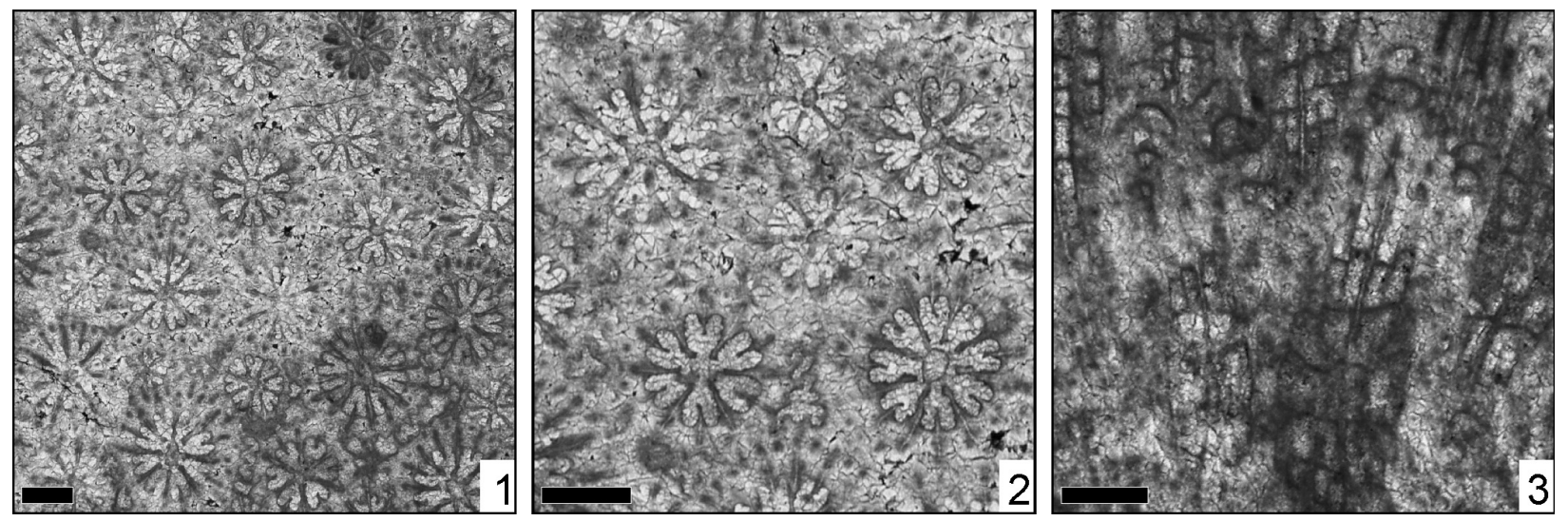

FIGURE 52. Stylina lamellosa Trautschold, 1886, MGB 83255. 1: transversal thin section. 2: transversal thin section, detail. 3: Iongitudinal thin section. Scale $1 \mathrm{~mm}$. 

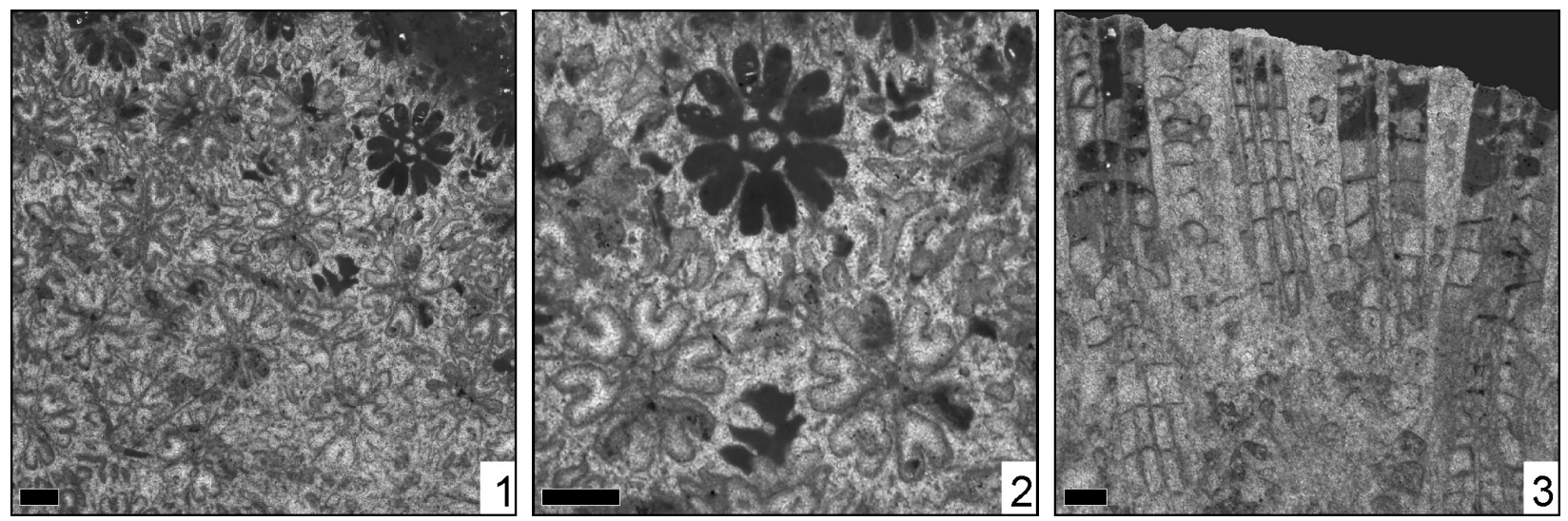

FIGURE 53. Stylina sp. 1, MGB 83306. 1: transversal thin section. 2: transversal thin section, detail. 3: Iongitudinal thin section. Scale $1 \mathrm{~mm}$.

Hauterivian of Ukraine (Krymskaya) Simferopolskij district, Alma river, Partizany [= Sably] (MGU).

\section{Stylina sp. 1 \\ Figure 53}

Material. MGB 83239, 83306, 83307, 83348; 5 thin sections.

Dimensions. See Table 46.

Remarks. The present material probably belongs to a new species. Most similar is Stylina digitiformis Achiardi, 1880, but it shows smaller corallite dimensions. From the Jurassic and Cretaceous, there are approximately 200 Stylina species formally described (Lathuilière, 1989; Löser, 2000). A comparison of new material with all these species

TABLE 46. Measurements of MGB 83306.

\begin{tabular}{ccccccc}
\hline & $\mathbf{n}$ & min-max & $\boldsymbol{\mu}$ & $\mathbf{s}$ & $\mathbf{c v}$ & $\boldsymbol{\mu \pm s}$ \\
\hline clmin & 30 & $1.75-2.28$ & 1.99 & 0.13 & 6.7 & $1.86-2.13$ \\
$\operatorname{clmax}$ & 30 & $2.05-2.84$ & 2.27 & 0.17 & 7.5 & $2.10-2.45$ \\
$\operatorname{ccd}$ & 30 & $2.32-3.99$ & 3.04 & 0.39 & 13.1 & $2.64-3.44$ \\
$\mathrm{~s}$ & 10 & $12-19$ & 12.9 & 2.23 & 17.3 & $11-15$ \\
\hline
\end{tabular}

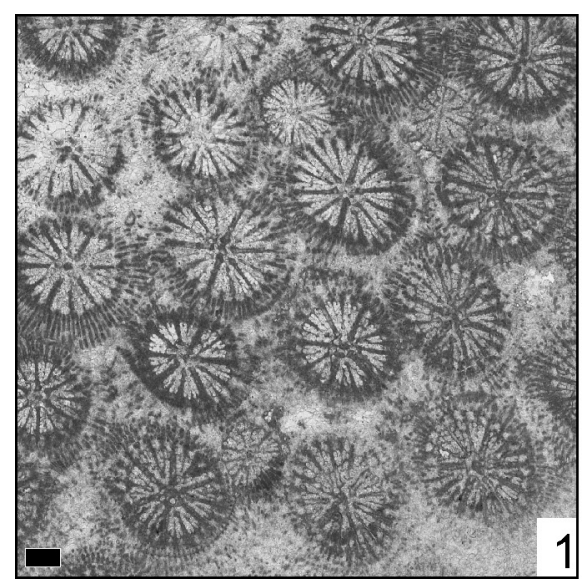

is difficult because the type material of many species is lost and morphometric data are rarely available from the literature.

Occurrence. Valanginian to Aptian of Mexico (Puebla) San Juan Raya (IGM nn).

\section{Stylina sp. 2}

Figure 54

Material. MGB 83320; 1 thin section.

Dimensions. See Table 47.

Remarks. The present material probably belongs to a new species. Stylina species with a hexameral septal symmetry and four septal cycles are very rare.

TABLE 47. Measurements of MGB 83320.

\begin{tabular}{ccccccc}
\hline & $\mathbf{n}$ & min-max & $\boldsymbol{\mu}$ & $\mathbf{s}$ & $\mathbf{c v}$ & $\boldsymbol{\mu \pm \mathbf { s }}$ \\
\hline clmin & 40 & $1.91-2.66$ & 2.32 & 0.22 & 9.5 & $2.10-2.55$ \\
clmax & 40 & $2.05-3.02$ & 2.57 & 0.23 & 9.2 & $2.33-2.80$ \\
ccd & 40 & $2.39-3.74$ & 3.01 & 0.41 & 13.6 & $2.60-3.42$ \\
s & $6+6+12+24$ & & & & & \\
\hline
\end{tabular}

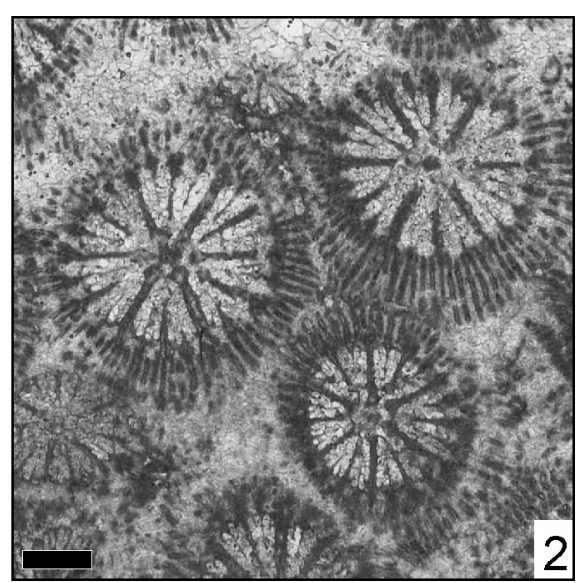

FIGURE 54. Stylina sp. 2, MGB 83320. 1: transversal thin section. 2: transversal thin section, detail. Scale $1 \mathrm{~mm}$. 
Occurrence. Tithonian of Japan (Fukushima-ken) Soma-gun area (TUM nn).

\section{Stylina sp. 3}

Figure 55

v 1897 Heliocoenia humberti Etallon, 1859; Ogilvie, p. 167 , pl. 18 , fig. 3

Material. MGB 83230, 83248, 83302, 83316, 83331; 6 thin sections.

Dimensions. See Table 48.

Remarks. The present material probably belongs also to a new species. Stylina species with a decameral septal symmetry and a so small corallite diameter are unknown. Identical with the present specimens is the specimen figured by Ogilvie (1897) as Heliocoenia humberti Etallon, 1859. This species has in fact a comparable calicular diameter, but belongs to Alloiteaucoenia, and is here considered a junior synonym of $A$. bernardina.

Occurrence. Tithonian to Lower Berriasian of Czech Republic (Moravia) Štramberk.
TABLE 48. Measurements of MGB 83248.

\begin{tabular}{ccccccc}
\hline & $\mathbf{n}$ & $\mathbf{m i n}-\mathbf{m a x}$ & $\boldsymbol{\mu}$ & $\mathbf{s}$ & $\mathbf{c v}$ & $\boldsymbol{\mu \pm \mathbf { s }}$ \\
\hline clmin & 30 & $0.73-0.93$ & 0.84 & 0.05 & 6.6 & $0.78-0.89$ \\
$\operatorname{clmax}$ & 30 & $0.80-1.04$ & 0.91 & 0.06 & 6.7 & $0.85-0.97$ \\
$\operatorname{ccd}$ & 30 & $0.73-1.20$ & 1.01 & 0.12 & 12.8 & $0.88-1.13$ \\
$\mathrm{~s}$ & $10+10$ & & & & & \\
\hline
\end{tabular}

\section{Stylina sp. 4}

Figure 56

Material. MGB 83389; 2 thin sections.

Dimensions. See Table 49.

Remarks. The material compares to Stylina sp. 3 but has clearly larger corallite dimensions.

Occurrence. Kimmeridgian of Germany (Bayern) Saal an der Donau (FLH 3210); France (Jura) Valfin (MNHN Beau6229). Lower Cenomanian (Dixoni Zone) of Spain (Cantabria, Santander) Cobreces, Luaña playa (BSPG 2007 V 203).
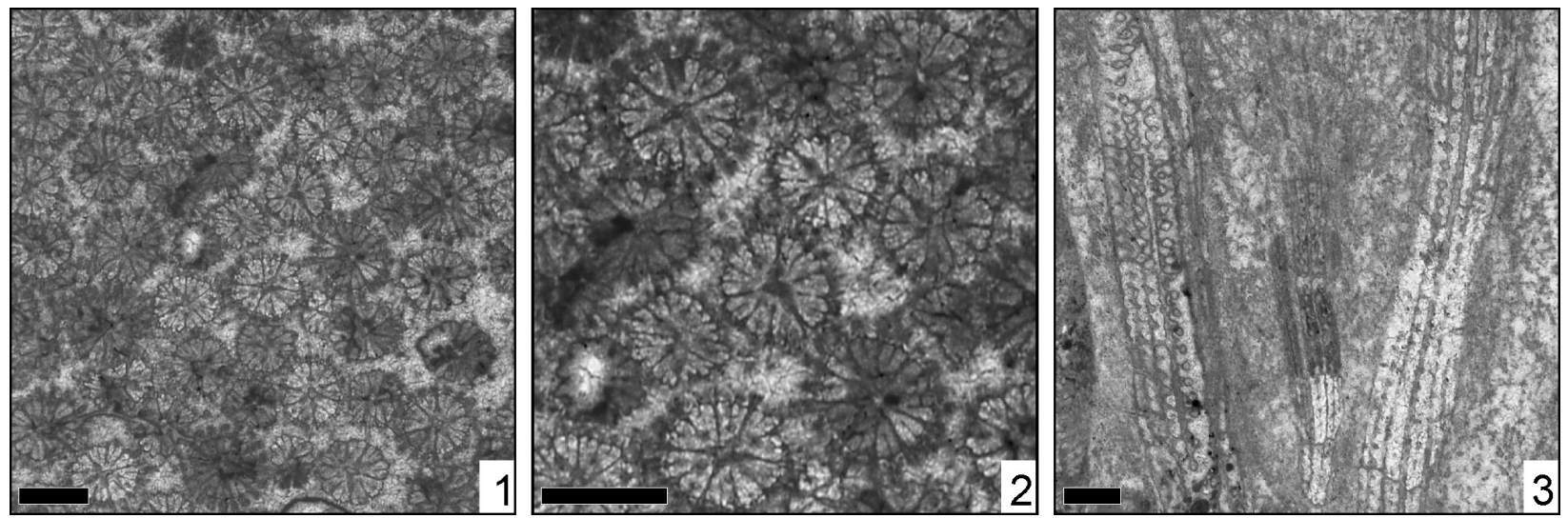

FIGURE 55. Stylina sp. 3, MGB 83248. 1: transversal thin section. 2: transversal thin section, detail. 3: Iongitudinal thin section. Scale $1 \mathrm{~mm}$.
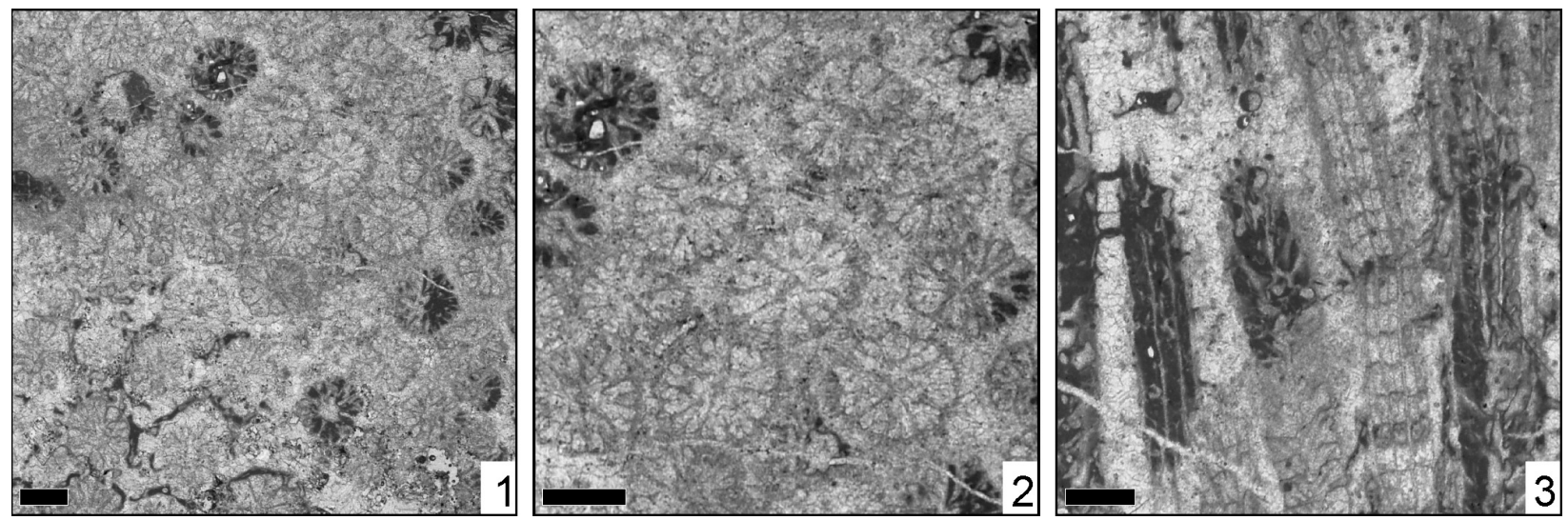

FIGURE 56. Stylina sp. 4, MGB 83389. 1: transversal thin section. 2: transversal thin section, detail. 3: Iongitudinal thin section. Scale $1 \mathrm{~mm}$. 
TABLE 49. Measurements of MGB 83389.

\begin{tabular}{ccccccc}
\hline & $\mathbf{n}$ & $\mathbf{m i n}-\mathbf{m a x}$ & $\boldsymbol{\mu}$ & $\mathbf{s}$ & $\mathbf{c v}$ & $\boldsymbol{\mu} \pm \mathbf{s}$ \\
\hline $\operatorname{clmin}$ & 20 & $0.98-1.31$ & 1.19 & 0.09 & 7.8 & $1.10-1.29$ \\
$\operatorname{clmax}$ & 20 & $1.17-1.57$ & 1.36 & 0.10 & 7.3 & $1.26-1.46$ \\
$\operatorname{ccd}$ & 20 & $1.06-1.79$ & 1.43 & 0.20 & 14.2 & $1.23-1.64$ \\
$\mathrm{~s}$ & $10+10$ & & & & & \\
\hline
\end{tabular}

\section{DISCUSSION}

\section{Sedimentological and Palaeogeographical Interpretation}

At a global scale, the Late Berriasian-Early Valanginian was affected by an eustatic sea-level fall (Haq et al., 1988; Haq, 2014), with a lowest sea-level reached at the beginning of the Late Valanginian. In the South Iberian Palaeomargin, a reactivation of the rifting has been proposed for this time, clearly affecting the Prebetic domain, within a syn-rift tectosedimentary stage (Vilas et al., 2001). As consequence of this extensional event, the Prebetic Jurassic platforms broke up, with development of tilted fault blocks, and a general increase in the subsidence rates, coeval with a major episode of terrigenous input, derived from the erosion of the Iberian Massif (García-Hernández, 1978, 2001).

In this context, a shallow mixed siliciclasticcarbonate platform was developed in the Prebetic during the Early Cretaceous. The facies and sedimentary structures, such as cross lamination and cross stratification, indicate high energy conditions in a very shallow marine environment. Palaeocurrents point to a consistent direction of transport from West to East. In these environments, some small coral patches developed, which were eroded by high energy events and incorporated into the calcarenite sediments forming cross-stratified bars.

\section{Faunal Composition}

The coral fauna distributes among nine superfamilies and covers many superfamilies known from this period, except the Cladocoroidea, Haplaraeoidea, Meandrophyllioidea, and Thamnasterioidea. The distribution of species among the present superfamilies varies (Figure 57). Most abundant are the Cyclolitoidea, a family that from the Middle Jurassic up to the whole Cretaceous always contributes with numerous species to coral faunas. In the Mid to Upper Jurassic dominate the Microsolenidae family, in the Lower Cretaceous the Latomeandridae and in the Upper Cretaceous the Cyclolitidae and Synastraeidae (Löser, 2016). The second species-rich superfamily, the Stylinoidea, is represented by three families, the Rhipidogyridae, the Stylinidae, and the Aulastraeoporidae, however, with only one species present. Abundance of the first two families is very characteristic for the uppermost Jurassic. Moderately common are the superfamilies Actinastreoidea, Amphiastreoidea, and Eugyroidea. The remaining superfamilies Heterocoenioidea, Misistelloidea, Montlivaltioidea and the informal Holocoenia-Group have only few species.

\section{Distribution}

Thirty-seven coral species found in the Sierra de Cazorla (out of 51) have a distribution outside of the study area. Of these 37 species, 11 occurred were hitherto only known from the Jurassic and Berriasian, nine species only from the Cretaceous after the base of the Valanginian, and 17 species were known from both Jurassic to Berriasian and from the Valanginian (Figure 58). The difference is irrelevant, and there is also not much coincidence between the ranges and the taxonomic position of the species. There is an obvious data gap from the middle Berriasian to Valanginian, illustrating the poor availability of coral faunas of this time span. When summarising the indications of the various

51 species

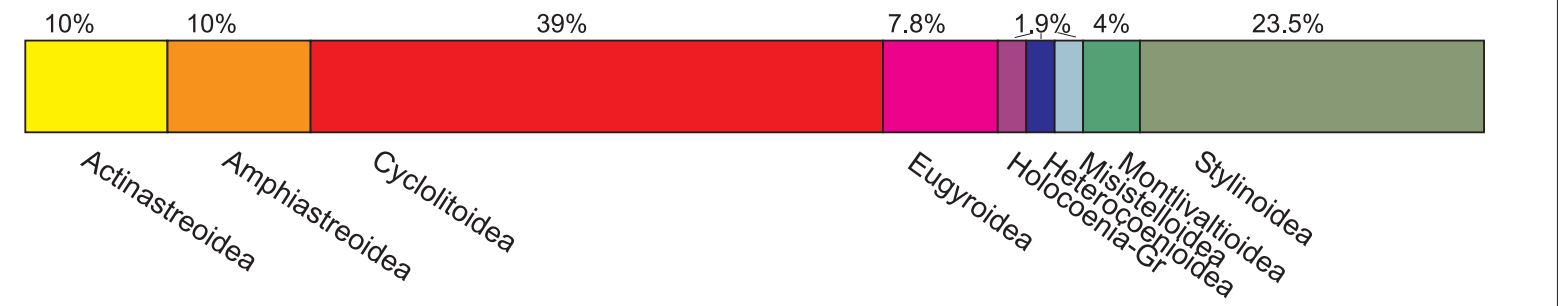

FIGURE 57. Distribution of coral species among superfamilies. 


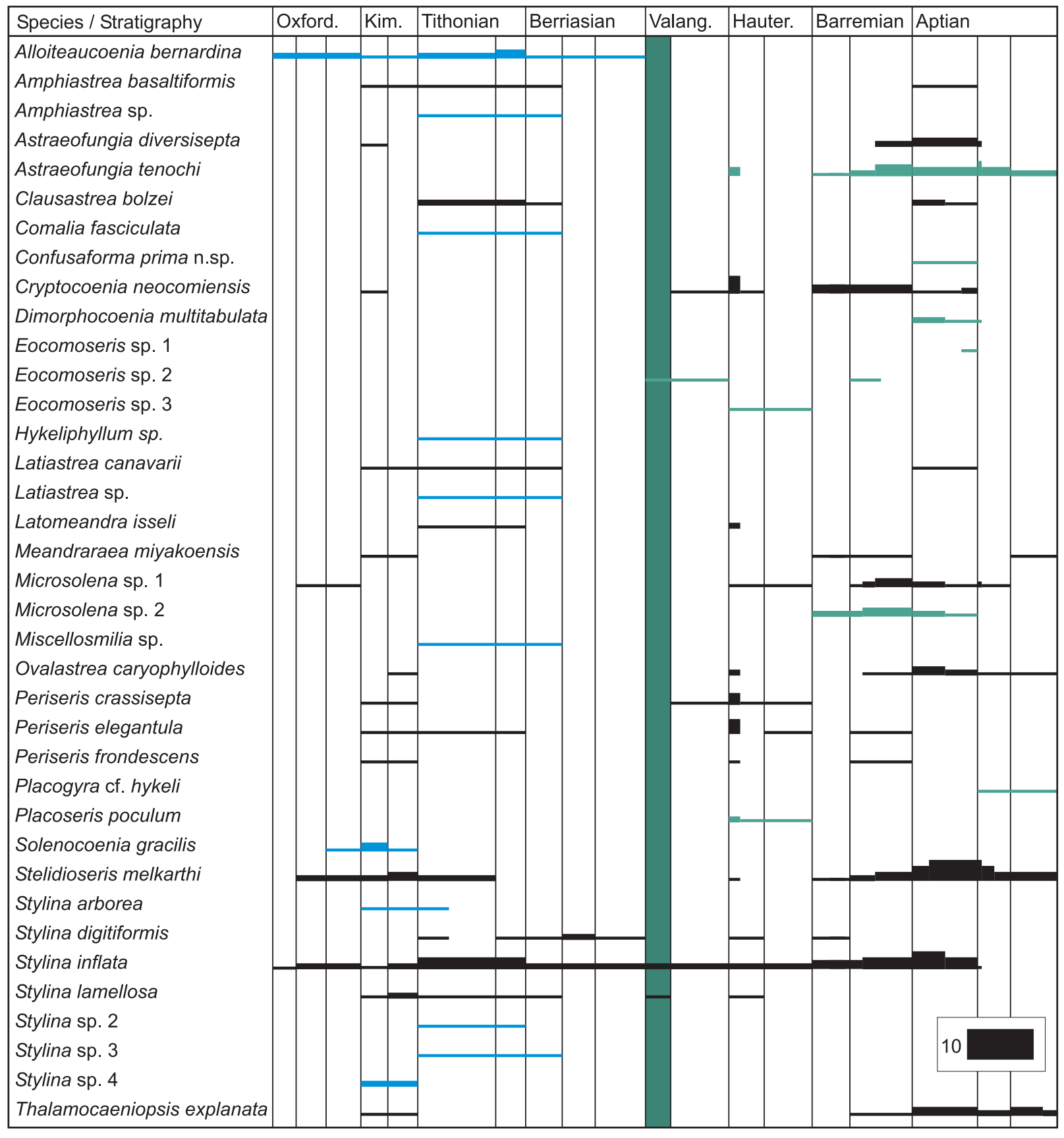

FIGURE 58. Distribution of species in localities outside of the study area. The thickness of bars corresponds to the number of localities where the species was found. Black bars correspond to species that occurred before and after the base of the Valanginian, light blue bars to species that occurred only in the Jurassic and Berriasian, and light green bars to species that are only known from the Valanginian on. The vertical dark green bar marks the age of the investigated coral fauna.

species, both aspects of the fauna can be observed: relationships to Kimmeridgian and Tithonian faunas, as well as to Hauterivian and upper Barremian to Aptian faunas (Figure 59).

A palaeobiogeographical analysis is very difficult and would bring low significant results because of the absence of comparable faunas. The correlation of palaeo-provinces (Figure 60) shows only four strong relationships, one to the Tithonian to lower Berriasian of the northern Tethys (the "Štramberk-type limestone"; Kolodziej, 2015a, $2015 b)$ with 10 species, to the Kimmeridgian of the 


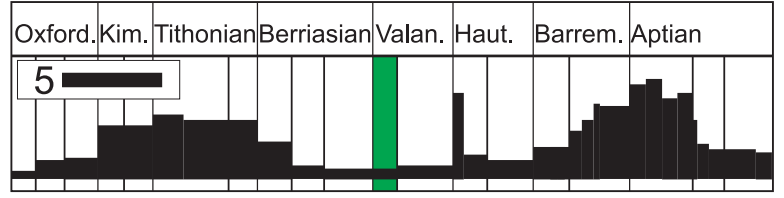

FIGURE 59. Summarised distribution of species in localities outside of the study area. The thickness of bars corresponds to the number of localities where the species was found. The vertical green bar marks the age of the investigated coral fauna.

northern Tethys (what corresponds to the Nattheim fauna of southwest Germany; Lauxmann, 1991), to the Hauterivian of the Paris Basin (Löser, 2013a), and to the Upper Valanginian to Lower Hauterivian of the Puebla Basin in Mexico (Löser et al., 2013). It must be admitted that all four faunas are extremely well studied and very rich in species. Provinces that show a lower correlation are equally less studied and/or less data are available.

\section{Evolution}

A clear pattern of a faunal turnover is not recognisable (Figure 61). There are four genera, which have their last occurrence in the study area; one genus is currently considered endemic to the area, and three genera have their first occurrence in the outcrop area. There are another three Jurassic genera that reached the Late Valanginian and one genus which reached into the Hauterivian.

When looking at the superfamilies and families and comparing the abundance in genera (Figure 62), it can be noticed that the time period of the Late Jurassic/Early Cretaceous is not so much faunal turnover than an increase in diversity. Except for the poorly understood Dermosmiliidae, all families that existed during the Late Jurassic reached at least the Valanginian/Hauterivian boundary. The Misistellidae and Rhipidogyridae became extinct, but all others survived at least into the Early Aptian. Therefore, the reduction in families was low. On the other hand, a remarkable increase in families can be observed throughout the whole Early Cretaceous. From the Valanginian to the Early Aptian, 16 new families appeared. This increase continued through the Cretaceous (Löser, 2016, fig. 6.1.5) and could be connected to several factors.

(1) The Cretaceous corals are much better investigated than the Jurassic ones. A systematic revision that exists for the Cretaceous corals (Löser, 2016) does not exist for the Jurassic corals.

(2) The distance in time from today to the Jurassic is greater than to the Cretaceous. Subse-

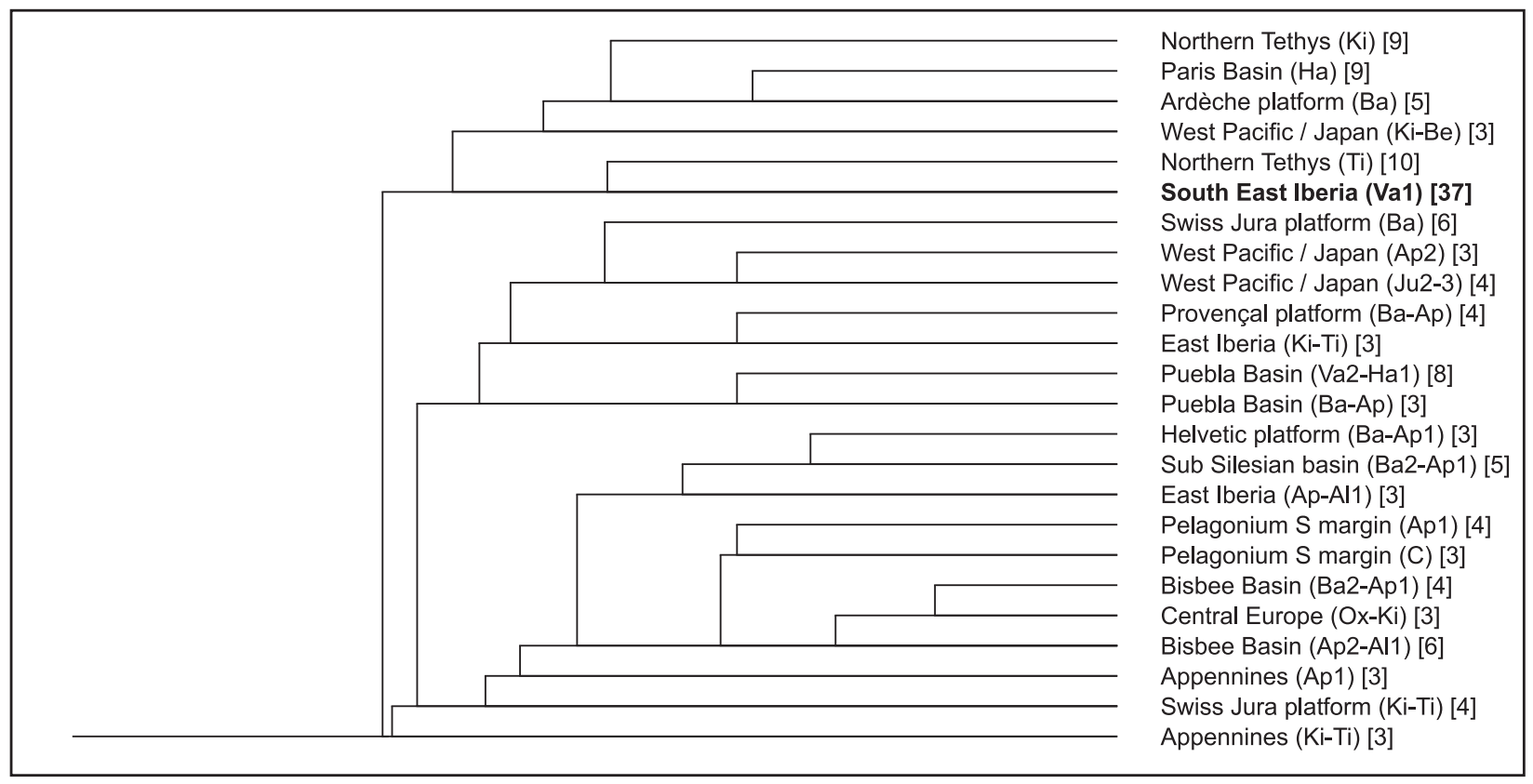

FIGURE 60. Correlation of the palaeo-provinces where species of the studied fauna occur. Only provinces with more than two species were included, and only the time period Oxfordian to Aptian was considered. The Correlation Ratio coefficient was applied, the graph is logarithmic. Abbreviations: Ju, Jurassic; Ox, Oxfordian; Ki, Kimmeridgian; Ti, Tithonian; C, Cretaceous; Be, Berriasian; Va, Valanginian; Ha, Hauterivian; Ba, Barremian; Ap, Aptian; Al, Albian. The number one indicate lower, the number two upper, in the Ju2-3 indicates Middle to Upper Jurassic. The numbers in brackets are the numbers of joint species. The study area is marked in bold letters. 


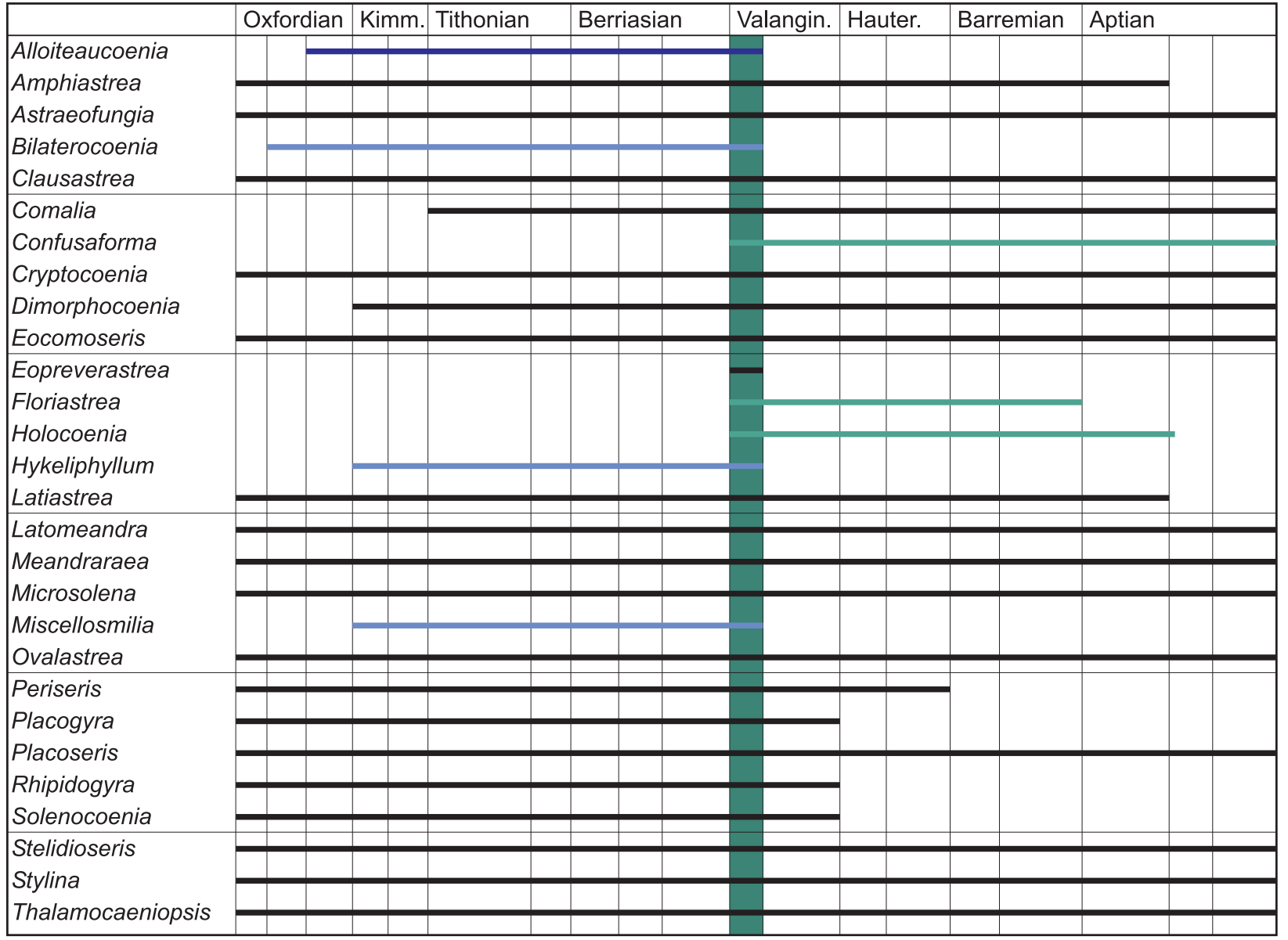

FIGURE 61. Critical stratigraphic ranges of the coral genera in the study area. Ranges after Löser (2016), but improved by newer data. Black lines indicate genera that occurred before and after the Early Valanginian, light blue lines genera that have their last occurrence in the outcrop area, light green lines genera that have the first occurrence in the outcrop area. The vertical green area shows the age of the studied fauna.

quently, there became more Jurassic faunas eroded than Cretaceous ones.

(3) The global sea-level was higher during the Cretaceous than the Jurassic. The greater area available for colonisation triggered the increase in diversity (also shown in Löser, 2016, figure 6.4.4B). However, the greenhouse climate during the Cretaceous resulted in more oceanic anoxic events. These events probably exerted a slight control on the evolution, but not on the abundance of genera (Löser, 2016, figure 6.4.4A).

(4) Generic richness was not controlled by reef abundance. Classical reef formation ended around the Jurassic/Cretaceous boundary due to a change in $\mathrm{Mg} / \mathrm{Ca}$ proportion of the seawater (Stanley and Hardy, 1998), not necessarily because corals were inhibited in the formation of their skeletons, but because of the absence of calcareous algae that would have bound the reefs together.

\section{CONCLUSIONS}

The revision of the Lower Valanginian coral fauna from the Puerto Llano section corresponding to the South Iberian Palaeomargin (Prebetic, Betic Cordillera) contributes to closing some of the gaps in knowledge in coral faunas of the Jurassic-Cretaceous transition. The taxonomic inventory shows that Berriasian and Valanginian coral faunas are dominated by Jurassic faunal elements. Several new genera and families appeared in the Valanginian, but their number is much lower compared to the genera and families that appeared during the Hauterivian and Barremian. Changes in the faunal composition of Cretaceous coral associations did not happen suddenly. It was a slow process that can only be deciphered by the systematic evaluation of well dated coral faunas. 


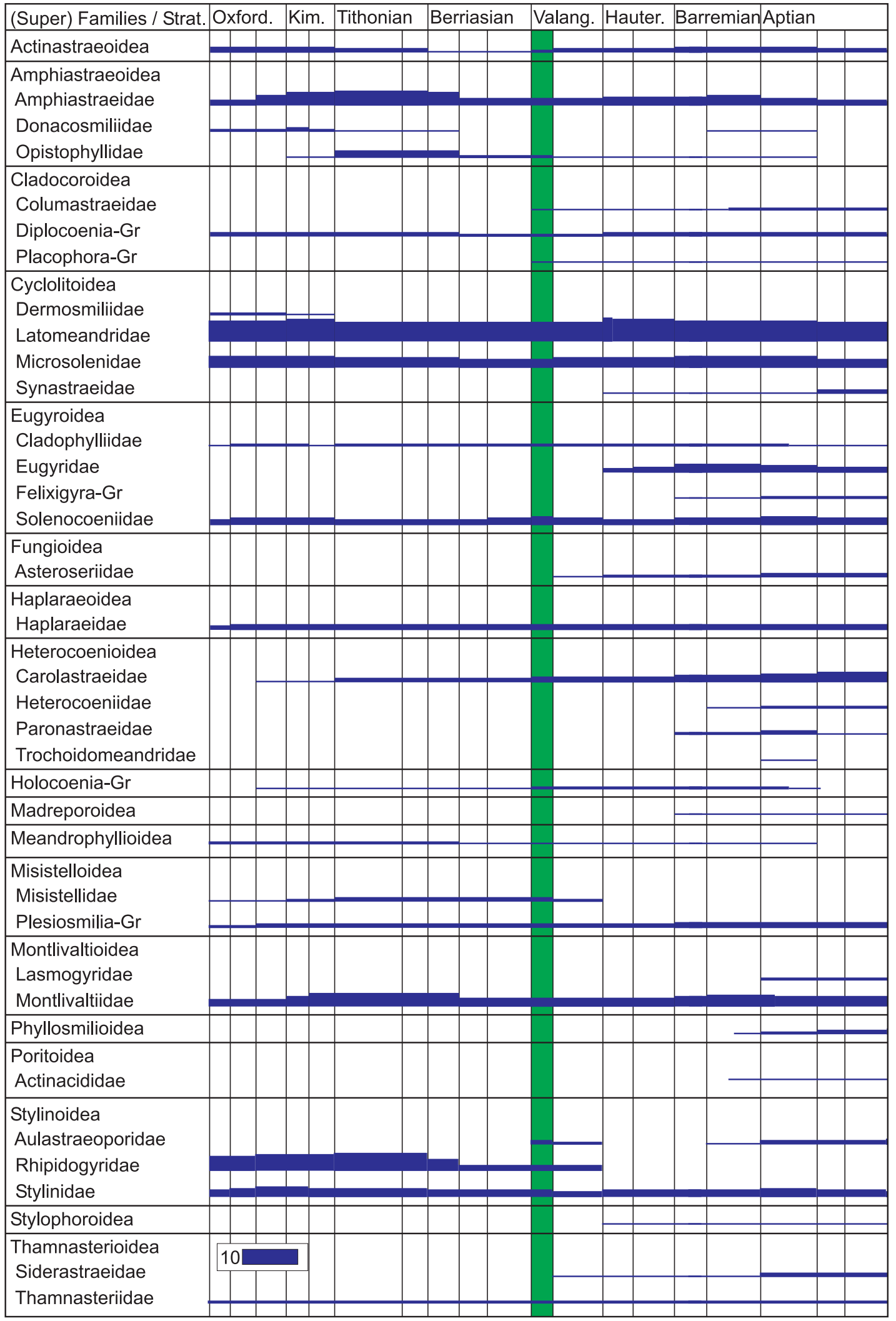

FIGURE 62. Critical stratigraphic ranges of coral families with a distribution in the time period Oxfordian to Aptian. Ranges after Löser (2016), but improved by newer data. The thickness of bars corresponds to the number of genera of the families. When a range is given for a superfamily, it collects only one family. The vertical green area shows the age of the studied fauna. 


\section{ACKNOWLEDGEMENTS}

This research is part of the results of the Project CGL2014-55274-P funded by the Secretaria de Estado de Investigación, Desarrollo e Innovación (Ministerio de Economía y Competitividad) of the Spanish Government. B. Kolodziej (Kraków) made helpful comments on the new genus Eopreverastrea. Preparation of thin sections by A. Orcí (Her- mosillo) is kindly acknowledged. A. Samaniego (Hermosillo) supported the study by measuring Jurassic corals in order to improve the database. English text correction by Proof-Reading-Services.com (Letchworth Garden City). The text was improved by comments of an anonymous reviewer and the handling editor.

\section{REFERENCES}

Achiardi, A. 1875. Coralli eocenici del Friuli. Parte 3. Atti della Società Toscana di Scienze naturali, 3:147-222.

Achiardi, A. 1880. Coralli giurassici dell'Italia settentrionale. Atti della Società Toscana di Scienze naturali, 4:233-310.

Alloiteau, J. 1952. Embranchement des coelentérés, p. 376-684. In Piveteau, J. (ed.), Traité de Paléontologie. Masson, Paris.

Alloiteau, J. 1954. Sur cinq genres nouveaux de madréporaires post-paléozoïques. Bulletin de la Société Géologique de France, (6), 3:873-887.

Alloiteau, J. 1957. Contribution à la Systématique des Madréporaires Fossiles. Centre National de la Recherche Scientifique, Paris.

Alloiteau, J. 1958. Monographie des Madréporaires fossiles de Madagascar. Annales Géologiques de Madagascar, 25:1-218.

Alloiteau, J. 1960. Sur le genre Clausastrea. Annales de Paléontologie, (Invertébrés), 46:3-46.

Anderson, E.J. 2004. The cyclic hierarchy of the "Purbeckian" Sierra del Pozo Section, Lower Cretaceous (Berriasian), southern Spain. Sedimentology, 51:455-477. https://doi.org/10.1111/j.1365-3091.2004.00632.x

Anonymous 2013. Pseudocoenia d'Orbigny, 1850 (Coelenterata, Scleractinia): proposed conservation of usage by the designation of a lectotype for the type species not approved. Bulletin of Zoological Nomenclature, 70:207-209. https://doi.org/10.21805/bzn.v70i3.a1

Arkadiev, V.V. and Bugrova, I.Yu. 1999. Facies of the Cretaceous (Berriasian) deposits from the river Belbek area (Southwestern Crimea). Facies, 40:71-80. https://doi.org/10.1007/bf02537469

Arkadiev, V.V., Guzhikov, A., Baraboshkin, E., Savelieva, J., Feodorova, A., Shurekova, O., Platonov, E., and Manikin, A. 2018. Biostratigraphy and magnetostratigraphy of the upper Tithonian - Berriasian of the Crimean Mountains. Cretaceous Research, 87:5-41. https://doi.org/10.1016/j.cretres.2017.07.011

Baron-Szabo, R.C. 1997. Die Korallenfazies der ostalpinen Kreide (Helvetikum: Allgäuer Schrattenkalk; Nördliche Kalkalpen: Brandenberger Gosau) Taxonomie, Palökologie. Zitteliana, 21:3-97.

Baron-Szabo, R.C. 2014. Scleractinian Corals from the Cretaceous of the Alps and Northern Dinarides with remarks on related taxa. Abhandlungen der Geologischen Bundesanstalt, 68:1-296.

Baron-Szabo, R.C. 2018. Scleractinian corals from the upper Berriasian of central Europe and comparison with contemporaneous coral assemblages. Zootaxa, 4383:1-98. https://doi.org/10.11646/zootaxa.4383.1.1

Baron-Szabo, R.C. and Steuber, T. 1996. Korallen und Rudisten aus dem Apt im tertiären Flysch des Parnass-Gebirges bei Delphi-Arachowa. Berliner geowissenschaftliche Abhandlungen, E18:3-75.

Baron-Szabo, R.C. and González León, C.M. 2003. Late Aptian-Early Albian corals from the Mural Limestone of the Bisbee Group (Tuape and Cerro de Oro areas), Sonora, Mexico, p. 187-225. In Scott, R.W. (ed.), Bob F. Perkins Memorial Volume, Special Publications in Geology. Gulf Coast Section SEPM Foundation, Houston. 
Baron-Szabo, R.C., Hamedani, A., and Senowbari-Daryan, B. 2003. Scleractinian corals from Lower Cretaceous deposits north of Esfahan (Central Iran). Facies, 48:199-216. https://doi.org/10.1007/bf02667539

Baron-Szabo, R.C. and Furrer, H. 2018. Korallen (Anthozoa), p. 113-143. In Kürsteiner, P. and Klug, C. (eds.), Fossilien im Alpstein. Kreide und Eozän der Nordostschweiz. Appenzeller Verlag, Schwellbrunn.

Beauvais, L. 1964. Étude stratigraphique et paléontologique des formations à madréporaires du Jurassique supérieur du Jura et de l'Est du Bassin de Paris. Mémoires de la Société géologique de France, 100:1-287.

Beauvais, L. 1966. Révision des madréporaires du Dogger de la collection Koby. Eclogae Geologicae Helvetiae, 59:989-1024.

Beauvais, L. 1976. Madréporaires du Jurassique (1:) Étude morphologique, taxonomique et phylogénétique du sous-ordre Amphiastraeida Alloiteau. Mémoires de la Société géologique de France, 126:1-42.

Beauvais, M. and M'Rabet, A. 1977. Les Madréporaires du Berriasien supérieur du Djebel Siouf (Axe Nord-Sud, Tunisie centrale). Notes du Service Géologique, 43:103-137.

Becker, E. 1875. Die Korallen der Nattheimer Schichten (1). Palaeontographica, 21:1-60.

Bover Arnal, T., Löser, H., Moreno Bedmar, J.A., Salas, R., and Strasser, A. 2012. Corals on the slope (Aptian, Maestrat Basin, Spain). Cretaceous Research, 37:43-64. https://doi.org/10.1016/j.cretres.2012.03.001

Castro, J.M., de Gea, G.A., Ruiz-Ortiz, P.A., and Nieto, L.M. 2008. Development of carbonate platforms on an extensional (rifted) margin. The Valanginian-Albian record of the Prebetic of Alicante (SE Spain). Cretaceous Research, 29:848-860. https://doi.org/10.1016/j.cretres.2008.05.012

Dietrich, W.O. 1926. Steinkorallen des Malms und der Unterkreide im südlichen DeutschOstafrika. Palaeontographica, (suppl. 7), 1:43-62.

Duncan, P.M. 1879. On the Upper Greensand coral fauna of Haldon, Devonshire. Quarterly Journal of the Geological Society of London, 35:89-97.

Eguchi, M. 1951. Mesozoic hexacorals from Japan. Science Reports of the Tohoku Imperial University, (2: Geology), 24:1-96.

Ehrenberg, C.G. 1834. Beiträge zur physiologischen Kenntnis der Corallenthiere im allgemeinen, und besonderen des rothen Meeres, nebst einem Versuche zur physiologischen Systematik derselben. Abhandlungen der Königlichen Akademie der Wissenschaften, (for 1832):225380.

Eliášová, H. 1973. Sous-famille Rhipidogyrinae Koby, 1905 (Hexacorallia) des calcaires de Štramberk (Tithonien, Tchécoslovaquie). Časopis pro mineralogii a geologii, 18:267-287.

Eliášová, H. 1975. Sous-ordre Amphiastraeina Alloiteau, 1952 (Hexacorallia) des calcaires de Štramberk (Tithonien, Tchécoslovaquie). Časopis pro mineralogii a geologii, 20:1-23.

Eliášová, H. 1976a. Familles Placosmiliidae Alloiteau, 1952 et Misistellidae nov.fam. (Hexacorallia) des calcaires de Štramberk. Časopis pro mineralogii a geologii, 21:337-347.

Eliášová, H. 1976b. Les coraux de l'ordre Hexanthiniaria Montanaro-Gallitelli, 1975, Zoantharia de Blainville, 1830, dans les calcaires de Štramberk (Tithonien, Tchécoslovaquie). Věstník Ústředního ústavu geologického, 51:357-366.

Eliášová, H. 1981. The Tithonian Reef of Štramberk Limestone (Czechoslovakia, West Carpathians). Časopis pro mineralogii a geologii, 26:113-124.

Eliášová, H. 2008. Corals from the Štramberk Limestone (Silesian Unit, Outer Western Carpathians, Czech Republik). Geología, 34:151-160.

Etallon, A. 1859. Études paléontologiques sur le Haut-Jura. Rayonnés du Corallien. Mémoires de la Société d'émulation du département du Doubs, 3:401-553.

Etallon, A. 1864. Classe des Polypes. Lethaea bruntrutana. Études paléontologiques et stratigraphiques sur le Jura bernois et en particulier sur les environs de Porrentruy (3). Neue Denkschriften der allgemeinen schweizerischen Gesellschaft für die gesammten Naturwissenschaften, 20:357-412.

Felix, J. 1891. Versteinerungen aus der mexicanischen Jura und Kreideformation. Palaeontographica, 37:140-194.

Felix, J. 1900. Über die Gruppe der Montlivaltiaceae. Sitzungsberichte der Naturforschenden Gesellschaft zu Leipzig, 1900:20-24.

Felix, J. 1909. Beiträge zur Kenntnis der Korallenfauna des Syrischen Cenoman. Beiträge zur Paläontologie und Geologie Österreich-Ungarns und des Orients, 22:169-175. 
Ferry, H. 1870. Polypiers nouveaux ou peu connus. Annales de l'Académie de Mâcon, 9:189206.

Fromentel, E. 1856. Note sur les polypiers fossiles de l'étage portlandien de la Haute-Saône. Bulletin de la Société Géologique de France, (2), 13:851-865.

Fromentel, E. 1857. Description des polypiers fossiles de l'étage Néocomien. Bulletin de la société des sciences historiques et naturelles de l'Yonne, 1-78.

Fromentel, E. 1861. Introduction à l'étude des polypiers fossiles. Mémoires de la Société d'émulation du Doubs, (3), 5:1-357.

Fromentel, E. 1863a. Monographie des polypiers jurassiques supérieurs (1:) Étage portlandien. Mémoires de la Société linnéenne de Normandie, 12:1-56.

Fromentel, E. 1863b. Zoophytes, terrain crétacé (4). Paléontologie française, 8:145-192.

Fromentel, E. 1879. Zoophytes, terrain crétacé (11). Paléontologie française, 8:481-512.

Fromentel, E. 1886. Zoophytes, terrain crétacé (15). Paléontologie française, 8:577-608.

García-Hernández, M. 1978. El Jurásico Terminal y el Cretácico Inferior en las Sierras de Cazorla y del Segura (Zona Prebética). Unpublished PhD Thesis, Universidad de Granada, Granada, Spain.

García-Hernández, M. 2001. El ciclo Valanginiense superior-Hauteriviense inferior en la Sierra de Segura: registro de una etapa de extensión cortical en el Cretácico Inferior de la Zona Prebética. Geotemas, 3:189-192.

Geyer, O.F. 1955. Beiträge zur Korallenfauna des Stramberger Tithon. Paläontologische Zeitschrift, 29:177-216.

Geyer, O.F. and Rosendahl, S. 1985. Stromatoporen, Korallen und Nerineen aus oberjurassischen und unterkretazischen Schichten des Präbetikums von Cazorla (Provinz Jaén, Spanien). Arbeiten aus dem Institut für Geologie und Paläontologie an der Universität Stuttgart, 82:161-179.

Gill, G.A. 1977. Essai de regroupement des Stylinides (hexacoralliaires) d'après la morphologie des bords internes de leurs septes. Mémoires du Bureau de Recherches Géologiques et Minières, 89:283-295.

Goldfuss, A. 1826. Petrefacta Germaniae. Arnz, Düsseldorf.

González León, O., Jeremiah, J., Schlagintweit, F., Bover Arnal, T., Moreno Bedmar, J.A., Mendoza Rosales, C.C., and Vega, F.J. 2015. Novel contributions and errata to the work "Morphology and ontogeny of the fossil lobster Meyeria magna M'Coy, 1849 (Astacidae: Mecochiridae) from the Lower Cretaceous (Lower Aptian) of Mexico, United Kingdom and Spain”. Neues Jahrbuch für Geologie und Paläontologie, Abhandlungen, 276, 3:323-334. https://doi.org/10.1016/j.cretres.2012.03.001

Götz, S., Löser, H., and Schmid, D.U. 2005. Reef development on a deepening platform: two Early Cretaceous coralgal patch reefs (Catí, Llàcova Formation, eastern Spain) compared. Cretaceous Research, 26:864-881. https://doi.org/10.1016/j.cretres.2005.05.008

Gregory, J.W. 1925. The collection of fossils and rocks from Somaliland made by Messrs B.K.N.Wyllie, B.Sc., F.G.S., and W.R.Smellie, D.Sc., F.R.S.Ed. Introduction and fossil corals. Monographs of the Geological Department of the Hunterian Museum, Glasgow University, 1:1-7.

Gregory, J.W. 1930. The fossil corals of Kenya colony collected by Miss McKinnon Wood. Monographs of the Geological Department of the Hunterian Museum, Glasgow University, 4:185-209.

Gréselle, B. and Pittet, B. 2010. Sea-level reconstructions from the Peri-Vocontian Zone (Southeast France) point to Valanginian glacio-eustasy. Sedimentology, 57:1640-1684. https://doi.org/10.1111/j.1365-3091.2010.01159.x

Hackemesser, M. 1936. Eine kretazische Korallenfauna aus Mittel-Griechenland und ihre paläobiologischen Beziehungen. Palaeontographica, (A), 84:1-97.

Haq, B.U., Handerbol, J., and Vail, P.R., 1988. Mesozoic and Cenozoic chronostratigraphy and eustatic cycles, p. 71-108. In Wilgus, C.K., Hasting, B.S., Kendall, C.G.S.C., Posamentier, H., Ross, C.A., and Van Wagoner, J.C. (eds.), Sea level changes-an integrated approach. SEPM Special Publication, 42. https://doi.org/10.2110/pec.88.01.0071

Haq, B.U. 2014. Cretaceous eustacy revisited. Global and Planetary Change, 113:44-58. https://doi.org/10.1016/j.gloplacha.2013.12.007

Husinec, A. and Jelaska, V. 2006. Relative sea-level changes recorded on an isolated carbonate platform: Tithonian to Cenomanian succession, southern Croatia. Journal of Sedimentary Research 76:1120-1136. https://doi.org/10.2110/jsr.2006.099 
Jerez-Mir, L. 1973. Geología de la Zona Prebética en la Transversal de Elche de la Sierra y Sectores Adyacentes (Provincias de Albacete y Murcia). Unpublished PhD Thesis, Universidad de Granada, Spain.

Jiménez de Cisneros, C. and Vera, J.A. 1993. Milankovitch ciclicity in Purbeck pertidal limestones of the Prebetic (Berriasian, southern Spain). Sedimentology, 40:513-537. https://doi.org/10.1111/j.1365-3091.1993.tb01348.x

Johnson, K.G. 2007. Reef-coral diversity in the Late Oligocene Antigua Formation and temporal variation of local diversity on Caribbean Cenozoic Reefs. Schriftenreihe der Erdwissenschaftlichen Kommissionen der Österreichischen Akademie der Wissenschaften, 17:471-491.

Kakizaki, Y., Ishikawa, T., Nagaishi, K., Tanimizu, M., Hasegawa, T., and Kano, A. 2012. Strontium isotopic ages of the Torinosu-type limestones (latest Jurassic to earliest Cretaceous, Japan): Implication for biocalcification event in northwestern Palaeo-Pacific. Journal of Asian Earth Sciences, 46:140-149. https://doi.org/10.1016/j.jseaes.2011.11.018

Koby, F. 1881. Monographie des polypiers jurassiques de la Suisse (2). Abhandlungen der Schweizerischen Paläontologischen Gesellschaft, 8:61-108.

Koby, F. 1887. Monographie des polypiers jurassiques de la Suisse (7). Abhandlungen der Schweizerischen Paläontologischen Gesellschaft, 14:353-400.

Koby, F. 1888. Monographie des polypiers jurassiques de la Suisse (8). Abhandlungen der Schweizerischen Paläontologischen Gesellschaft, 15:401-456.

Koby, F. 1889. Monographie des polypiers jurassiques de la Suisse (9). Abhandlungen der Schweizerischen Paläontologischen Gesellschaft, 16:457-586.

Koby, F. 1896. Monographie des polypiers crétacés de la Suisse (1). Abhandlungen der Schweizerischen Paläontologischen Gesellschaft, 22:1-28.

Koby, F. 1897. Monographie des polypiers crétacés de la Suisse (2). Abhandlungen der Schweizerischen Paläontologischen Gesellschaft, 23:29-62.

Koby, F. 1898. Monographie des polypiers crétacés de la Suisse (3). Abhandlungen der Schweizerischen Paläontologischen Gesellschaft, 24:63-100.

Koby, F. 1905. Description de la faune jurassique du Portugal, polypiers du Jurassique supérieur. Communicações da Commissão do Servico Geológico de Portugal, 1-167 pp.

Kolodziej, B. 2003. Scleractinian corals of suborders Pachythecaliina and Rhipidogyrina: discussion on similarities and description of species from Štramberk-type limestones, Polish Outer Carpathians. Annales Societatis Geologorum Poloniae, 73:193-217.

Kolodziej, B. 2015a. Geological context and age of the Štramberk-type limestone from the Polish Outer Carpathians. Neues Jahrbuch für Geologie und Paläontologie, Abhandlungen, 276:173-179. https://doi.org/10.1127/njgpa/2015/0476

Kolodziej, B. 2015b. Corals of the Štramberk-type limestone from Poland: Taxonomic and palaeoecological aspects. Neues Jahrbuch für Geologie und Paläontologie, Abhandlungen, 276:181-199. https://doi.org/10.1127/njgpa/2015/0477

Kuzmicheva, E.I. 1960. Hexacorals, p.125-141. In Menner, V.V. (ed.), Atlas nizhnemelovoj fauny Severnogo Kavkaza i Kryma. Nauka, Moskva. [in Russian]

Kuzmicheva, E.I. 1963. New species of Early Valanginian solitary scleractinians from the Mountain Crimea. Paleontologicheskij zhurnal, 3:18-26. [in Russian]

Kuzmicheva, E.I. 1966. Stratigraphical and facial distribution of hexacorals (scleractinians) in the Neocomian of the Mountain Crimea. Prirodnye i trudovye resursy levoberezhnoj Ukrainy i ikh ispolzovanie, 58-63. [in Russian]

Kuzmicheva, E.I. 2002. Skeletal morphology, systematics and evolution of the Scleractinia. Trudy Paleontologicheskogo instituta, 286:1-211. [in Russian]

Lamarck, J.B.P. 1816. Histoire Naturelle des Animaux sans Vertèbres. Verdière, Paris.

Lamouroux, J.V.F. 1821. Exposition Méthodique des Genres de l'Ordre des Polypiers. Agasse, Paris.

Lathuilière, B. 1989. Répertoire Objectif des Coraux Jurassiques. Presses universitaires, Nancy.

Lathuilière, B. 1990. Periseris: scléractiniaire colonial jurassique. Révision structurale et taxinomie de populations bajociennes de l'Est de la France. Geobios, 23:33-55. https://doi.org/10.1016/0016-6995(90)80017-a

Lauxmann, U. 1991. Revision der oberjurassischen Korallen von Württemberg (SWDeutschland), exclusive Fungiina. Palaeontographica, (A), 219:107-175.

Liao, Wei-hua and Xia, Jin-bao 1994. Mesozoic and Cenozoic scleractinian corals from Tibet. Palaeontologia Sinica (Zhongguo-gushengwu-zhi), 184:1-252. [in Chinese] 
Löser, H. 1987. Zwei neue Gattungen der Korallen aus der Sächsischen und Böhmischen Oberkreide. Věstník Ústředního ústavu geologického, 62:233-237.

Löser, H. 1989. Die Korallen der sächsischen Oberkreide (1:) Hexacorallia aus dem Cenoman. Abhandlungen des Staatlichen Museums für Mineralogie und Geologie zu Dresden, 36:88154.

Löser, H. 1994. La faune corallienne du mont Kassenberg à Mülheim-sur-la-Ruhr (Bassin crétacé de Westphalie, Nord Ouest de l'Allemagne). Coral Research Bulletin, 3:1-93.

Löser, H. 2000. Repertoire of Species. Catalogue of Cretaceous Corals, 1:1-137.

Löser, H. 2001. Le site de Vallières (département de l'Aube, France): résultats préliminaires sur des coraux de l'Hauterivien inférieur (Crétacé). Bulletin annuel de l'Association géologique de l'Aube, 22:39-53.

Löser, H. 2004. PaleoTax - a database program for palaeontological data. Computer \& Geosciences, 30:513-521. https://doi.org/10.1016/j.cageo.2004.03.009

Löser, H. 2005. Stratigraphy of Cretaceous coral genera. Neues Jahrbuch für Geologie und Paläontologie, Abhandlungen, 238:231-277. https://doi.org/10.1127/njgpa/238/2006/231

Löser, H. 2006. Barremian corals from San Antonio Texcala, Puebla, Mexico - A review of the type material of Felix 1891. Boletín del Instituto Geológico de México, 114:1-68.

Löser, H. 2007. Case 3386: Pseudocoenia d'Orbigny, 1850 (Coelenterata, Scleractinia): proposed conservation of usage by the designation of a lectotype for the type species. Bulletin of Zoological Nomenclature, 64:79-82.

Löser, H. 2008. Early Cretaceous coral faunas from East Africa (Tanzania, Kenya; Late Valanginian-Aptian) and revision of the Dietrich collection (Berlin, Germany). Palaeontographica, 285:23-75. https://doi.org/10.1127/pala/285/2008/23

Löser, H. 2009. Morphology, taxonomy and distribution of the Early Cretaceous coral genus Holocoenia (Scleractinia) and its first record in the Caribbean. Revista Mexicana de Ciencias Geológicas, 26:93-103.

Löser, H. 2012a. Revision of Actinastrea, the most common Cretaceous coral genus. Paläontologische Zeitschrift, 86, 1:15-22. https://doi.org/10.1007/s12542-011-0110-4

Löser, H. 2012b. Intraspecific variation in the genus Stelidioseris (family Actinastraeidae, suborder Archeocaeniina, order Scleractinia; Jurassic-Cretaceous). Geologica Belgica, 15:382-387.

Löser, H. 2013a. Revision of the Hauterivian (Early Cretaceous) corals of the Paris Basin, France: a work in progress. Bulletin d'Information des Géologues du Bassin de Paris, 501:17-24.

Löser, H. 2013b. The Cretaceous corals from the Bisbee Group (Sonora; Late Barremian - Early Albian): genus Stelidioseris (Actinastraeidae). Paleontología Mexicana, 63:79-89.

Löser, H. 2014. 3. Korallen / 3. Corals. Geologica Saxonica, 60:17-56.

Löser, H. 2015. The Cretaceous corals from the Bisbee Group (Sonora; Late Barremian-Early Albian): Solenocoeniidae. Paleontología mexicana, 4:13-24.

Löser, H. 2016. Systematic part. Catalogue of Cretaceous Corals, 4:1-710.

Löser, H. and Ferry, S. 2006. Coraux du Barrémien du Sud de la France (Ardèche et Drôme). Geobios, 39:469-489. https://doi.org/10.1016/j.geobios.2005.03.005

Löser, H. and Mori, K. 2002. The Jurassic corals from Japan in the Tohoku University Museum collection. Bulletin of the Tohoku University Museum, 2:77-110.

Löser, H. and Minor, K. 2007. Palaeobiogeographic aspects of Late Barremian to Late Albian coral faunas from Northern Mexico (Sonora) and the southern USA (Arizona, Texas). Neues Jahrbuch für Geologie und Paläontologie, Abhandlungen, 245:193-218. https://doi.org/10.1127/0077-7749/2007/0245-0193

Löser, H. and Raeder, M. 1995. Aptian/Albian coral assemblages of the Helicon Mountains (Boeotia, Greece): palaeontological, palaeoecological and palaeogeographical aspects. Coral Research Bulletin, 4:37-63.

Löser, H., Arias, C., and Vilas, L. 2019. Upper Valanginian to Lower Hauterivian coral faunas from the Sierra Larga (Prebetic zone, SE Spain). Neues Jahrbuch für Geologie und Paläontologie, Abhandlungen, 292:1-32. https://doi.org/10.1127/njgpa/2019/0820

Löser, H., Barattolo, F., Badia, S., Chikhi-Aouimeur, F., Dhondt, A., Erlich, R.N., Fözy, I., Geister, J., Hiss, M., Kolodziej, B., Leloux, J., Lewy, Z., Minor, K.P., Mitchell, S., Moosleitner, G., Peza, L., Remane, J., Romano, R., Sikharulidze, G.Y., Sinnyovski, D., Steuber, T., Tröger, K.-A., Turnšek, D., Vecchio, E., Vilella i Puig, J., and Zítt, J. 2002. List of Citations. Catalogue of Cretaceous Corals, 2:1-784. 
Löser, H., Barattolo, F., Badia, S., Chikhi-Aouimeur, F., Dhondt, A., Erlich, R.N., Fözy, I., Geister, J., Hiss, M., Kolodziej, B., Leloux, J., Lewy, Z., Madhavaraju, J., Minor, K.P., Mitchell, S., Moosleitner, G., Niebuhr, B., Peza, L., Remane, J., Romano, R., Sanders, D., Sikharulidze, G.Y., Sinnyovski, D., Steuber, T., Tröger, K.-A., Turnšek, D., Vecchio, E., Vilella I Puig, J., and Zítt, J. 2005. List of Localities. Catalogue of Cretaceous Corals, 3:1-366.

Löser, H., Stemann, Th.A., and Mitchell, S.F. 2009. Oldest scleractinian fauna from Jamaica (Hauterivian, Benbow Inlier). Journal of Paleontology, 83:333-349. https://doi.org/10.1666/08-060.1

Löser, H., Castro, J.M., and Nieto, L.M. 2013. Late Albian scleractinian corals from the Prebetic Zone (SE Spain). Palaeontographica, 301:1-62. https://doi.org/10.1127/pala/301/2013/1

Löser, H. and Bilotte, M. 2017. Taxonomy of a platy coral association from the Late Cenomanian of the southern Corbières (Aude, France). Annales de Paléontologie, 103:3-17. https://doi.org/10.1016/j.annpal.2016.10.005

Löser, H., García-Barrera, P., Mendoza Rosales, C.C. and Ortega-Hernández, J. 2013. Corals from the Early Cretaceous (Barremian - Early Albian) of Puebla (Mexico) - Introduction and family Stylinidae. Revista Mexicana de Ciencias Geológicas, 30, 2:385-403.

Löser, H., Werner, W., and Darga, R. 2013d. A Middle Cenomanian coral fauna from the Northern Calcareous Alps (Bavaria, Southern Germany) - new insights into the evolution of Mid-Cretaceous corals. Zitteliana, A53:37-76.

Löser, H. and Heinrich, M. 2018. New coral genera and species from the Rußbach/Gosau area (Late Cretaceous; Austria). Palaeodiversity, 11:127-149. https://doi.org/10.18476/pale.11.a7

Löser, H., Steuber, T, and Löser, C. 2018. Early Cenomanian coral faunas from Nea Nikopoli (Kozani, Greece; Cretaceous). Carnets de Géologie / Notebooks on Geology, 18:23-121. https://doi.org/10.4267/2042/66094

Löser, H., Heinrich, M., and Schuster, U. 2019. Korallen von Rußbach und Gosau (ConiacSanton; Österreich). CPress, Dresden.

Martín-Chivelet, J., Berástegui, X., Rosales, I., Vilas, L., Vera, J.A., Caus, E., Gräfe, K.U., Segura, M., Puig, C., Mas, R., Robles, S., Floquet, M., Quesada, S., Ruiz-Ortiz, P.A., Fregenal-Martínez, M.A., Salas, R., García, A., Martín-Algarra, A., Arias, C., Meléndez, N., Chacón, B., Molina, J.M., Sanz, J.L., Castro, J.M., García-Hernández, M., Carenas, B., García-Hidalgo, J., Gil, J., and Ortega, F. 2002. Cretaceous, p. 255-292. In Gibbons, W. and Moreno, T. (eds.), Geology of Spain. Geological Society of London, London. https://doi.org/10.1144/gospp.12

Marzouk, L. and Ben Youssef, M. 2008. Relative sea-level changes of the Lower Cretaceous deposits in the Chotts Area of Southern Tunisia. Turkish Journal of Earth Sciences, 17:835845.

Matthews, S.C. 1973. Notes on open nomenclature and on synonymy lists. Palaeontology, 16:713-719.

Melnikova, G.K., Roniewicz, E., and Loeser, H. 1993. New microsolenid coral genus Eocomoseris (Scleractinia, Early Lias-Cenomanian). Annales Societatis Geologorum Poloniae, 63:3-12.

Michelin, H. 1841. Iconographie Zoophytologique. Description par localités et terrains des polypiers fossiles de France (1). Bertrand, Paris.

Michelin, H. 1843. Iconographie Zoophytologique. Description par localités et terrains des polypiers fossiles de France (3). Bertrand, Paris.

Milne Edwards, H. 1857. Histoire Naturelle des Coralliaires ou Polypes Proprement dits (1+2). Librairie encyclopédique de Roret, Paris.

Milne Edwards, H. and Haime, J. 1848. Observations sur les polypiers de la famille des astréides. Comptes rendus hebdomadaires des séances de l'Académie des Sciences, 27:465-469

Milne Edwards, H. and Haime, J. 1849a. Mémoire sur les polypiers appartenant à la famille des oculinides, au groupe intermédiaire des Pseudoastréides et à la famille des Fongides. Comptes rendus hebdomadaires des séances de l'Académie des Sciences, 29:67-73.

Milne Edwards, H. and Haime, J. 1849b. Recherches sur les polypiers (4:) Monographie des Astreides (2:) Astréens (1-3). Annales de Sciences Naturelles, (3), 11:233-312.

Milne Edwards, H. and Haime, J. 1851. A monograph of the British fossil corals (2:) Corals from the oolitic formations. Palaeontographical Society Monographs, 5:74-146.

Morycowa, E. 1964. Hexacoralla des couches de Grodziszcze (Néocomien Carpathes). Acta Palaeontologica Polonica, 9:1-114. 
Morycowa, E. 1971. Hexacorallia et Octocorallia du Crétacé inférieur de Rarau (Carpathes orientales roumaines). Acta Palaeontologica Polonica, 16:1-149.

Morycowa, E. 1974. Hexacorallia d'un bloc exotique de calcaire tithonique à Wo|zniki près de Wadowice (Carpathes polonaises occidentales). Acta Geologica Polonica, 24:457-484.

Morycowa, E. and Decrouez, D. 1993. Description de quelques coraux des calcaires urgoniens du domaine Delphino-Helvétique (Bornes, Haute-Savoie, France), première partie. Revue de Paléobiologie, 12:203-215.

Morycowa, E. and Marcopoulou-Diacantoni, A. 1997. Cretaceous scleractinian corals from the Parnassos area (Central Greece) (Preliminary note). Bulletin of the Geological Society of Greece, 30:249-273.

Morycowa, E. and Masse, J.P. 2009. Lower Cretaceous Microsolenina (Scleractinia) from Provence (Southern France). Annales Societatis Geologorum Poloniae, 79:97-140.

Ogilvie, M.M. 1897. Die Korallen der Stramberger Schichten. Palaeontographica, (suppl. 2), 7:74-282.

Ohga, H., Kolodziej, B., Nose, M., Schmid, D.U., Takayanagi, H., and Iryu, Y. 2013. Sedimentary facies and biofacies of the Torinosu Limestone in the Torinosu area, Kochi Prefecture, Japan. Island Arc, 22:1-20.

Oppenheim, L.P. 1930. Die Anthozoen der Gosauschichten in den Ostalpen. Privately published, Berlin.

Orbigny, A. 1849. Note sur les Polypiers Fossiles. Masson, Paris.

Orbigny, A. 1850. Prodrôme de Paléontologie Stratigraphique Universelle des Animaux Mollusques et Rayonnés. Masson, Paris.

Orbigny, A. 1851. Cours Élémentaire de Paléontologie (3:) Polypiers ou Zoophytes. Masson, Paris.

Pocta, F. 1887. Die Anthozoen der boehmischen Kreideformation. Abhandlungen der Königlichen Boehmischen Gesellschaft der Wissenschaften, (7), 2:1-60.

Prever, P.L. 1909a. Anthozoa. La fauna coralligena del Cretaceo dei Monti d'Ocre nell'Abruzzo Aquilano. Memorie descrittive della carta geologica d'Italia, 5:51-147.

Prever, P.L. 1909b. Coralli giurassici del Gran Sasso d'Italia. Atti della Reale Accademia di Scienze di Torino, 44:986-1000.

Reig Oriol, J. 1994. Madreporarios Cretácicos de Cataluña. Privately published, Barcelona.

Reyeros de Castillo, M.M. 1983. Corales de algunas formaciones cretacicas del estado de Oaxaca. Paleontología mexicana, 47:1-67.

Reyeros Navarro, M.M. 1963. Corales del Cretacico inferior de San Juan Raya, Estado de Puebla. Paleontología mexicana, 17:1-21.

Ricci, C., Lathuilière, B., and Rusciadelli, G. 2018. Coral communities, zonation and paleoecology of an Upper Jurassic reef complex (Ellipsactinia limestones, central Apennines, Italy). Rivista italiana di paleontologia e stratigrafia, 124:433-508.

Roemer, F.A. 1841. Die Versteinerungen des Norddeutschen Kreidegebirges. Hahn'schen Hofbuchhandlung, Hannover.

Roniewicz, E. 1976. Les scléractiniaires du Jurassique supérieur de la Dobrogea centrale Roumanie. Palaeontologia Polonica, 34:17-121.

Roniewicz, E. 2008. Kimmeridgian-Valanginian reef corals from the Moesian platform from Bulgaria. Annales Societatis Geologorum Poloniae, 78:91-134.

Roniewicz, E. 2011. Early Norian (Triassic) corals from the Northern Calcareous Alps, Austria, and the intra-Norian faunal turnover. Acta Palaeontologica Polonica, 56:401-428.

Ruiz-Ortiz, P.A., Castro, J.M., Arias, C., Vilas, L., Martín-Chivelet, J., de Gea, G.A., Molina, J.M., Nieto, L.M., Reolid, M., Aguado, R., Quijano, M.L., and Coruña, F. 2019. 5.3 The South Iberian Continental Margin, p. 190-205. In Quesada, C. and Oliveira, J.T. (eds.), The Geology of Iberia: A Geodynamic Approach. Regional Geology Reviews. Springer Nature Switzerland Cham. https://doi.org/10.1007/978-3-030-10519-8

Schöllhorn, E. 1998. Geologie und Paläontologie des Oberapt im Becken von Organyà (Nordspanien). Coral Research Bulletin, 6:1-139.

Sikharulidze, G.Ya. 1979. Albian corals near the Tskhanar village (Western Georgia). Trudy Akademija Nauk Gruzinskoj SSR, Geologiceskij Institut, 63:1-49. [in Russian]

Sikharulidze, G.Ya. 1985. Hexacorals from the Urgonian facies of the Dzirul Massif and its northern frame. Trudy Akademija Nauk Gruzinskoj SSR, Geologiceskij Institut, 59:1-110. [in Russian] 
Stanley, S.M. and Hardie, L.A. 1998. Secular oscillations in the carbonate mineralogy of reefbuilding and sediment-producing organisms driven by tectonically forced shifts in seawater chemistry. Palaeogeography, Palaeoclimatology, Palaeoecology, 144:3-19.

Tomás, S., Löser, H., and Salas, R. 2008. Low-light and nutrient-rich coral assemblages in an Upper Aptian carbonate platform of the southern Maestrat Basin (Iberian Chain, eastern Spain). Cretaceous Research, 29:509-534. https://doi.org/10.1016/j.cretres.2007.09.001

Tomes, R.F. 1893. Observations on the affinities of the genus Astrocoenia. Quarterly Journal of the Geological Society of London, 49:569-573.

Toula, F. 1880. Geologische Untersuchungen im westlichen Theile des Balkans und in den angrenzenden Gebiete (9:) Von Ak-Palanka über Nis, Leskovac und die Rui Planina bei Trn nach Pirot. Sitzungsberichte der Mathematisch-Naturwissenschaftliche Classe der Kaiserlichen Akademie der Wissenschaften, (1), 81:188-266.

Trautschold, H.A. 1886. Le Néocomien de Sably en Crimée. Trudy Imperatorskogo S.Peterburgskago Obshchestva Estestvoispytatelej, 15:119-129.

Turnšek, D. and Buser, S. 1974. Spodnjekredne korale, hidrozoji in hetetide z Banjske Planote in Trnovskega Gozda. Razprave Slovenska akademija znanosti in umetnosti, (4), 17, 2:81-124. [In Slovenian]

Turnšek, D. and Mihajlovic, M. 1981. Lower Cretaceous Cnidarians from eastern Serbia. Razprave Slovenska akademija znanosti in umetnosti, (4), 23:1-54.

Vašíček, Z., Michalík, J., and Reháková, D. 1994. Early Cretaceous stratigraphy, palaeogeography and life in Western Carpathians. Beringeria, 10:1-168.

Vašíček, Z. and Skupien, P. 2016. Tithonian-early Berriasian perisphinctoid ammonites from the Štramberk Limestone at Kotouc Quarry near Štramberk, Outer Western Carpathians (Czech Republic). Cretaceous Research, 64:12-29. https://doi.org/10.1016/j.cretres.2016.03.005

Vašíček, Z. and Skupien, P. 2019. New Tithonian and Lower Berriasian aptychi of Štramberk Limestone from the Kotouč Quarry (Outer Western Carpathians, Czech Republic). Paläontologische Zeitschrift, 93:37-48. https://doi.org/10.1007/s12542-018-0418-4

Vera, J.A., García-Hernández, M., López-Garrido, A.C., Comas, M.C., Ruiz-Ortiz, P.A., and Martín-Algarra, A. 1982. El Cretácico de las Cordilleras Béticas, p. 512-632. In García, A. (ed.), El Cretácico de España. Editorial Universidad Complutense, Madrid, Spain.

Vera, J.A. 2001. Evolution of the South Iberian Continental Margin, p. 109-143. In Ziegler, P.A., Cavazza, W., Robertson, A.H.F., and Crasquin-Soleau, S. (eds.), Peri-Tethys Memoir 6: Peri-Tethyan Rift/Wrench Basins and Passive Margins. Mémoires du Museum National d'Histoire Naturelle, 186. Paris.

Vera, J.A. 2004. Zonas Externas Béticas, p. 354-389. In Vera, J.A. (coord.), Geología de España. SGE-IGME, Madrid, Spain.

Vilas, L., Dabrio, C.J., Peláez, J.R., and García-Hernández, M. 2001. Dominios sedimentarios generados durante el período extensional Cretácico Inferior entre Cazorla y Hellín (Béticas Externas). Su implicación en la estructura actual. Revista de la Sociedad Geológica de España, 14:113-122.

Wells, J.W. 1932. Corals of the Trinity Group of the Commanchean of central Texas. Journal of Paleontology, 6:225-256.

Wells, J.W. 1936. The nomenclature and type species of some genera of recent and fossil corals. American journal of science, (5), 31:97-134.

Zell, P., Stinnesbeck, W., and Hering, F. 2016. A coral-rich unit of Berriasian (Early Cretaceous) age in the Sierra Madre Oriental of northeastern Mexico. Journal of South American Earth Sciences, 69:91-102. https://doi.org/10.1016/j.jsames.2016.03.010 